\title{
EXISTENCE AND STABILITY OF SCHRÖDINGER SOLITONS ON NONCOMPACT MANIFOLDS
}

\author{
DAVID BORTHWICK, ROLAND DONNINGER, ENNO LENZMANN, AND JEREMY L. MARZUOLA
}

\begin{abstract}
We consider the focusing nonlinear Schrödinger equation on a large class of rotationally symmetric, noncompact manifolds. We prove the existence of a solitary wave by perturbing off the flat Euclidean case. Furthermore, we study the stability of the solitary wave under radial perturbations by analyzing spectral properties of the associated linearized operator. Finally, in the $L^{2}$-critical case, by considering the Vakhitov-Kolokolov criterion (see also results of Grillakis-Shatah-Strauss), we provide numerical evidence showing that the introduction of a nontrivial geometry destabilizes the solitary wave in a wide variety of cases, regardless of the curvature of the manifold. In particular, the parameters of the metric corresponding to standard hyperbolic space will lead to instability consistent with the blow-up results of Banica-Duyckaerts (2015). We also provide numerical evidence for geometries under which it would be possible for the Vakhitov-Kolokolov condition to suggest stability, provided certain spectral properties hold in these spaces.
\end{abstract}

\section{INTRODUCTION}

The focusing nonlinear Schrödinger equation

$$
i \partial_{t} u(t, x)+\Delta_{x} u(t, x)+u(t, x)|u(t, x)|^{p-1}=0, \quad p>1,
$$

for an unknown $u: \mathbb{R} \times \mathbb{R}^{d} \rightarrow \mathbb{C}$, is a prototypical dispersive partial differential equation that arises in various situations in physics, e.g., in nonlinear optics or as an effective equation in many particle quantum mechanics. We refer the reader to the standard monograph [66] for the general background. It is a classical result that in the parameter range $1<p<1+\frac{4}{d-2}$ $(d \geq 2$, no upper bound if $d=2)$, Eq. (1.1) possesses solitary waves or solitons, i.e., solutions of the form

$$
u_{\alpha}^{*}(t, x)=e^{i \alpha^{2} t} Q_{\mathbb{R}^{d}, \alpha}(x), \quad \alpha>0,
$$

where the profile function $Q_{\mathbb{R}^{d}, \alpha} \in H^{1}\left(\mathbb{R}^{d}\right)$ is radial, smooth, positive, and exponentially decaying, see [22, 65, 23, 33, 34, 7, 9]. Note that $Q_{\mathbb{R}^{d}, \alpha}$ satisfies the elliptic equation

$$
-\Delta Q_{\mathbb{R}^{d}, \alpha}+\alpha^{2} Q_{\mathbb{R}^{d}, \alpha}-Q_{\mathbb{R}^{d}, \alpha}\left|Q_{\mathbb{R}^{d}, \alpha}\right|^{p-1}=0 .
$$

The upper bound $p=1+\frac{4}{d-2}$ has an interpretation in terms of scaling. Observe that if $u$ is a solution to Eq. (1.1), then so is the rescaled function

$$
u_{\lambda}(t, x):=\lambda^{-\frac{2}{p-1}} u\left(t / \lambda^{2}, x / \lambda\right)
$$

for any $\lambda>0$. When measured in homogeneous Sobolev spaces, the rescaled solution satisfies

$$
\left\|u_{\lambda}(t, \cdot)\right\|_{\dot{H}^{s}\left(\mathbb{R}^{d}\right)}=\lambda^{\frac{d}{2}-s-\frac{2}{p-1}}\left\|u\left(t / \lambda^{2}, \cdot\right)\right\|_{\dot{H}^{s}\left(\mathbb{R}^{d}\right)}
$$

R.D. is supported by the Austrian Science Fund FWF, Project P 30076.

J.L.M. was supported in part by NSF Applied Math Grant DMS-1312874 and NSF CAREER Grant DMS-1352353. 
and thus, if $p=1+\frac{4}{d-2}$, the $\dot{H}^{1}\left(\mathbb{R}^{d}\right)$-norm is invariant under the scaling. This is called the energy-critical case. Similarly, $p=1+\frac{4}{d}$ is called the mass-critical or $L^{2}$-critical case as it leaves the $L^{2}\left(\mathbb{R}^{d}\right)$-norm invariant. The scaling symmetry also shows that it is enough to consider $\alpha=1$ in Eq. (1.2), and in this case, the solution $u_{1}^{*}$ is unique [22, 51, 47] and called the ground state.

The ground state has a variational characterization which is closely related to stability properties. More precisely, this refers to the notion of orbital stability. Roughly speaking, $u_{1}^{*}$ is orbitally stable if any solution $u$ that starts out close to $u_{1}^{*}$ stays close to $u_{1}^{*}$ for all times, modulo symmetries of the equation. It is known that the ground state $u_{1}^{*}$ is orbitally stable in the $L^{2}$-subcritical case $p<1+\frac{4}{d}$ and unstable otherwise [8, 15, 70, 61, 72, 36, 35, 37].

The stronger notion of asymptotic stability of $u_{1}^{*}$ refers to the property that all solutions $u$ starting out sufficiently close to $u_{1}^{*}$ converge to $u_{1}^{*}$ as $t \rightarrow \infty$, modulo symmetries of the equation. Proving asymptotic stability is challenging as it presupposes a detailed knowledge of the spectrum of the nonself-adjoint operator that arises upon linearization of the equation at the ground state. Unfortunately, the mathematical understanding of this operator is still unsatisfactory and one has to rely in part on numerical evidence. Consequently, asymptotic stability is known only in special cases or under suitable spectral assumptions, see e.g. 63, 64, 13, 57, 25, 26, 14, 55, 29, 59, 5, 28, 6, 52, for an incomplete selection of available results.

1.1. Main results. In the present paper we change the geometry and investigate the existence of solitary waves and their spectral stability for Schrödinger equations on manifolds. More precisely, let $\mathbb{M}^{d}=(0, \infty) \times{ }_{A} \mathbb{S}^{d-1}, d \geq 2$, be a warped product manifold with warping function $A: \mathbb{R} \rightarrow \mathbb{R}$ and $\mathbb{S}^{d-1}$ equipped with the standard round metric, see e.g. 56. For the sake of concreteness, we use the stereographic projection $\psi: \mathbb{R}^{d-1} \rightarrow \mathbb{S}^{d-1}$,

$$
\psi(y):=\left(\frac{2 y}{|y|^{2}+1}, \frac{|y|^{2}-1}{\left|y^{2}\right|+1}\right),
$$

to parametrize the sphere. Then we have

$$
\partial_{a} \psi^{j}(y) \partial_{b} \psi_{j}(y)=\frac{4}{\left(|y|^{2}+1\right)^{2}} \delta_{a b}
$$

and the components $g_{j k}$ of the Riemannian metric on the warped product $\mathbb{M}^{d}$ are given by

$$
g_{j k}(r, y)=\delta_{1 j} \delta_{1 k}+\frac{4 A(r)^{2}}{\left(|y|^{2}+1\right)^{2}} \delta_{j k}\left(1-\delta_{1 j} \delta_{1 k}\right)
$$

for $j, k \in\{1,2, \ldots, d\}$. We also remark that the sectional curvatures of $\mathbb{M}^{d}$ are given by

$$
K\left(\partial_{r}, \partial_{y^{a}}\right)(r, y)=-\frac{A^{\prime \prime}(r)}{A(r)}, \quad K\left(\partial_{y^{a}}, \partial_{y^{b}}\right)(r, y)=\frac{1-A^{\prime}(r)^{2}}{A(r)^{2}}\left(1-\delta_{a b}\right),
$$

for $a, b \in\{1,2, \ldots, d-1\}$, see [56].

Hypothesis 1.1. We make the following assumptions on the warping function $A$.

- $A: \mathbb{R} \rightarrow \mathbb{R}$ is smooth and odd with $A^{\prime}(0)=1$.

- $A(r) \gtrsim r$ for all $r>0$.

- There exists a constant $V_{0, d} \in \mathbb{R}$ such that

$$
\frac{d-1}{2} \frac{A^{\prime \prime}(r)}{A(r)}+\frac{(d-1)(d-3)}{4}\left[\frac{A^{\prime}(r)^{2}}{A(r)^{2}}-\frac{1}{r^{2}}\right]=V_{0, d}\left[1+O\left(\langle r\rangle^{-2}\right)\right]
$$


for all $r>0$.

Remark 1.2. A classical example covered by Hypothesis 1.1 is $A(r)=\sinh (r)$ so that $\mathbb{M}^{d}$ is the hyperbolic space.

As usual, we denote by $\left(g^{j k}\right)$ the matrix inverse of $\left(g_{j k}\right)$ and $\operatorname{det} g$ is the determinant of the latter matrix. Explicitly, we have

$$
\sqrt{\operatorname{det} g(r, y)}=A(r)^{d-1}\left(\frac{2}{|y|^{2}+1}\right)^{d-1},
$$

and for the inner product $(\cdot \mid \cdot)_{L^{2}\left(\mathbb{M}^{d}\right)}$ on $L^{2}\left(\mathbb{M}^{d}\right)$ we obtain the expression

$$
(f \mid g)_{L^{2}\left(\mathbb{M}^{d}\right)}=\int_{0}^{\infty} \int_{\mathbb{R}^{d-1}} f(r, y) \overline{g(r, y)} A(r)^{d-1}\left(\frac{2}{|y|^{2}+1}\right)^{d-1} d y d r
$$

Furthermore, the Laplace-Beltrami operator $\Delta_{\mathbb{M}^{d}}$ on $\mathbb{M}^{d}$ is given by

$$
\Delta_{\mathbb{M}^{d}}:=\frac{1}{\sqrt{\operatorname{det} g}} \partial_{j}\left(\sqrt{\operatorname{det} g} g^{j k} \partial_{k}\right),
$$

where $\partial_{1}=\partial_{r}$ and $\partial_{j}=\partial_{y^{j-1}}$ for $j=2,3, \ldots, d$. We consider the focusing nonlinear Schrödinger equation

$$
i \partial_{t} u(t, \cdot)+\Delta_{\mathbb{M}^{d}} u(t, \cdot)+u(t, \cdot)|u(t, \cdot)|^{p-1}=0
$$

on $\mathbb{M}^{d}$ for a function $u: \mathbb{R} \times \mathbb{M}^{d} \rightarrow \mathbb{C}$. Our first result concerns the existence of solitary waves or solitons.

Theorem 1.3 (Existence of solitary waves). Assume Hypothesis 1.1 and $1<p<1+\frac{4}{d-2}$ (no upper bound in the case $d=2$ ). Then there exists an $\alpha_{0}>0$ such that for any $\alpha \geq \alpha_{0}$, there exists a real-valued function $Q_{\mathbb{M}^{d}, \alpha} \in C^{2}\left(\mathbb{M}^{d}\right)$ for which $u_{\alpha}^{*}: \mathbb{R} \times \mathbb{M}^{d} \rightarrow \mathbb{C}$, given by

$$
u_{\alpha}^{*}(t, r, y):=e^{i \alpha^{2} t} Q_{\mathbb{M}^{d}, \alpha}(r, y),
$$

is a solution to Eq. (1.4) for all $t \in \mathbb{R}$. More precisely, we have

$$
Q_{\mathbb{M}^{d}, \alpha}(r, y)=\alpha^{\frac{2}{p-1}}\left(\frac{r}{A(r)}\right)^{\frac{d-1}{2}}\left[Q_{\mathbb{R}^{d}, 1}\left(\alpha r e_{1}\right)+\rho_{\alpha}\left(\alpha r e_{1}\right)\right],
$$

where $\rho_{\alpha} \in C^{2}\left(\mathbb{R}^{d}\right)$ satisfies $\left\|\rho_{\alpha}\right\|_{H^{2}\left(\mathbb{R}^{d}\right)}+\left\|\rho_{\alpha}\right\|_{L^{\infty}\left(\mathbb{R}^{d}\right)} \lesssim \alpha^{-1}$ for all $\alpha \geq \alpha_{0}$. In particular, $Q_{\mathbb{M}^{d}, \alpha}$ is radial.

Remark 1.4. The soliton profile on the manifold is a perturbation of the Euclidean profile. The heuristic behind this fact is that for large $\alpha$ the soliton is supposed to concentrate near the origin and one expects the curvature to become negligible. This effect is quantified in Theorem 1.3 .

We continue by investigating the linear stability of the solitary wave from Theorem 1.3 . By plugging the ansatz $u(t, r, y)=e^{i \alpha^{2} t}\left[Q_{\mathbb{M}^{d}, \alpha}(r, y)+w(t, r, y)\right]$ into Eq. (1.4), one obtains, upon dropping the nonlinear terms, the evolution equation

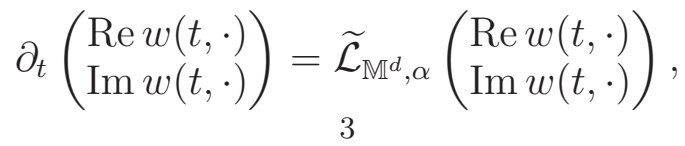


with the operator

$$
\widetilde{\mathcal{L}}_{\mathbb{M}^{d}, \alpha}:=\left(\begin{array}{cc}
0 & \widetilde{\mathcal{L}}_{\mathbb{M}^{d}, \alpha,-} \\
-\widetilde{\mathcal{L}}_{\mathbb{M}^{d}, \alpha,+} & 0
\end{array}\right),
$$

where

$$
\begin{aligned}
\widetilde{\mathcal{L}}_{\mathbb{M}^{d}, \alpha,-} & :=-\Delta_{\mathbb{M}^{d}}+\alpha^{2}-\left|Q_{\mathbb{M}^{d}, \alpha}\right|^{p-1} \\
\widetilde{\mathcal{L}}_{\mathbb{M}^{d}, \alpha,+} & :=-\Delta_{\mathbb{M}^{d}}+\alpha^{2}-p\left|Q_{\mathbb{M}^{d}, \alpha}\right|^{p-1} .
\end{aligned}
$$

Evidently, the linear stability of $u_{\alpha}^{*}$ is encoded in the spectral properties of (a closed realization of) the operator $\widetilde{\mathcal{L}}_{\mathbb{M}^{d}, \alpha}$. We restrict our attention to the radial case and consider $\widetilde{\mathcal{L}}_{\mathbb{M}^{d}, \alpha}$ on the space $L_{\text {rad }}^{2}\left(\mathbb{M}^{d}, \mathbb{C}^{2}\right)$ with domain

$$
\mathcal{D}\left(\widetilde{\mathcal{L}}_{\mathbb{M}^{d}, \alpha}\right):=\left\{\left(f_{1}, f_{2}\right) \in C_{c}^{\infty}\left(\mathbb{M}^{d}, \mathbb{C}^{2}\right): f_{1}, f_{2} \text { radial }\right\} .
$$

Accordingly, we equip the scalar operators $\widetilde{\mathcal{L}}_{\mathbb{M}^{d}, \alpha, \pm}$ with the domains

$$
\mathcal{D}\left(\widetilde{\mathcal{L}}_{\mathbb{M}^{d}, \alpha, \pm}\right):=\left\{f \in C_{c}^{\infty}\left(\mathbb{M}^{d}\right): f \text { radial }\right\} .
$$

Theorem 1.5 (Structure of the spectrum of the linearized operator). Assume Hypothesis 1.1 and $1<p<1+\frac{4}{d-2}$ (no upper bound in the case $d=2$ ). There exists an $\alpha_{0}>0$ such that for any $\alpha \geq \alpha_{0}$, the operator $\widetilde{\mathcal{L}}_{\mathbb{M}^{d}, \alpha}: \mathcal{D}\left(\widetilde{\mathcal{L}}_{\mathbb{M}^{d}, \alpha}\right) \subset L_{\text {rad }}^{2}\left(\mathbb{M}^{d}, \mathbb{C}^{2}\right) \rightarrow L_{\text {rad }}^{2}\left(\mathbb{M}^{d}, \mathbb{C}^{2}\right)$ is closable. Its closure $\mathcal{L}_{\mathbb{M}^{d}, \alpha}$ has the following properties:

- The spectrum of $\mathcal{L}_{\mathbb{M}^{d}, \alpha}$ is a subset of $\mathbb{R} \cup i \mathbb{R}$.

- If $\lambda \in \sigma\left(\mathcal{L}_{\mathbb{M}^{d}, \alpha}\right)$ then $-\lambda \in \sigma\left(\mathcal{L}_{\mathbb{M}^{d}, \alpha}\right)$.

- The essential spectrum 11 of $\mathcal{L}_{\mathbb{M}^{d}, \alpha}$ is given by

$$
\sigma_{e}\left(\mathcal{L}_{\mathbb{M}^{d}, \alpha}\right)=\left\{z \in \mathbb{C}: \operatorname{Re} z=0,|\operatorname{Im} z| \geq \alpha^{2}+V_{0, d}\right\} .
$$

- The set $\sigma\left(\mathcal{L}_{\mathbb{M}^{d}, \alpha}\right) \backslash \sigma_{e}\left(\mathcal{L}_{\mathbb{M}^{d}, \alpha}\right)$ is free of accumulation points and consists of eigenvalues with finite algebraic multiplicities.

- We have $0 \in \sigma_{p}\left(\mathcal{L}_{\mathbb{M}^{d}, \alpha}\right)$ and

$$
\operatorname{ker} \mathcal{L}_{\mathbb{M}^{d}, \alpha}=\left\langle\left(\begin{array}{c}
0 \\
Q_{\mathbb{M}^{d}, \alpha}
\end{array}\right)\right\rangle
$$

For $p \neq 1+\frac{4}{d}$ we obtain a very clear picture concerning the linear stability which is analogous to the Euclidean situation.

Theorem 1.6 (Spectral stability in the noncritical case). Assume Hypothesis 1.1 and $1<$ $p<1+\frac{4}{d-2}$ (no upper bound in the case $d=2$ ). Then there exists an $\alpha_{0}>0$ such that for all $\alpha \geq \alpha_{0}$ the following holds.

- If $p \neq 1+\frac{4}{d}$, the algebraic multiplicity of $0 \in \sigma_{p}\left(\mathcal{L}_{\mathbb{M}^{d}, \alpha}\right)$ equals 2 .

- If $p<1+\frac{4}{d}$, there are no positive eigenvalues of $\mathcal{L}_{\mathbb{M}^{d}, \alpha}$.

- If $p>1+\frac{4}{d}$, there exists precisely one positive eigenvalue $\lambda_{\alpha} \in \sigma_{p}\left(\mathcal{L}_{\mathbb{M}^{d}, \alpha}\right)$ and the eigenvalues $\pm \lambda_{\alpha} \in \sigma_{p}\left(\mathcal{L}_{\mathbb{M}^{d}, \alpha}\right)$ are simple.

\footnotetext{
${ }^{1}$ There are various (in general inequivalent) definitions of the essential spectrum of a closed operator. For us, the essential spectrum is the part of the spectrum that is invariant under relatively compact perturbations, see Definition 4.1 and Remark 4.2 below.
} 
In the critical case $p=1+\frac{4}{d}$, the stability of the solitary wave is more involved and depends on finer properties of the underlying geometry. The corresponding condition is formulated in terms of $\mathcal{L}_{\mathbb{M}^{d}, \alpha,+}^{-1}$, where $\mathcal{L}_{\mathbb{M}^{d}, \alpha,+}$ is the closure of $\widetilde{\mathcal{L}}_{\mathbb{M}^{d}, \alpha,+}$.

Theorem 1.7 (Spectral stability in the critical case). Assume Hypothesis 1.1 and $1<p<$ $1+\frac{4}{d-2}$ (no upper bound in the case $d=2$ ). Then there exists an $\alpha_{0}>0$ such that for all $\alpha \geq \alpha_{0}$ the operator $\widetilde{\mathcal{L}}_{\mathbb{M}^{d}, \alpha,+}: \mathcal{D}\left(\widetilde{\mathcal{L}}_{\mathbb{M}^{d}, \alpha,+}\right) \subset L_{\text {rad }}^{2}\left(\mathbb{M}^{d}\right) \rightarrow L_{\text {rad }}^{2}\left(\mathbb{M}^{d}\right)$ is essentially self-adjoint and its closure $\mathcal{L}_{\mathbb{M}^{d}, \alpha,+}$ is bounded invertible. If $p=1+\frac{4}{d}$ then for all $\alpha \geq \alpha_{0}$ the following holds:

- If $\left(\mathcal{L}_{\mathbb{M}^{d}, \alpha,+}^{-1} Q_{\mathbb{M}^{d}, \alpha} \mid Q_{\mathbb{M}^{d}, \alpha}\right)_{L^{2}\left(\mathbb{M}^{d}\right)}>0$ then $\mathcal{L}_{\mathbb{M}^{d}, \alpha}$ has precisely one positive eigenvalue $\lambda_{\alpha}$ and the eigenvalues $\pm \lambda_{\alpha} \in \sigma_{p}\left(\mathcal{L}_{\mathbb{M}^{d}, \alpha}\right)$ are simple.

- If $\left(\mathcal{L}_{\mathbb{M}^{d}, \alpha,+}^{-1} Q_{\mathbb{M}^{d}, \alpha} \mid Q_{\mathbb{M}^{d}, \alpha}\right)_{L^{2}\left(\mathbb{M}^{d}\right)} \leq 0$ then $\mathcal{L}_{\mathbb{M}^{d}, \alpha}$ has no positive eigenvalues.

Remark 1.8. If $\partial_{\alpha} Q_{\mathbb{M}^{d}, \alpha}$ is sufficiently smooth and belongs to the domain of $\mathcal{L}_{\mathbb{M}^{d}, \alpha,+}$, the (in)stability condition in Theorem 1.7 can be simplified. Indeed, by differentiating

$$
-\Delta_{\mathbb{M}^{d}} Q_{\mathbb{M}^{d}, \alpha}+\alpha^{2} Q_{\mathbb{M}^{d}, \alpha}-Q_{\mathbb{M}^{d}, \alpha}\left|Q_{\mathbb{M}^{d}, \alpha}\right|^{p-1}=0
$$

with respect to $\alpha$ we find

$$
\mathcal{L}_{\mathbb{M}^{d}, \alpha,+} \partial_{\alpha} Q_{\mathbb{M}^{d}, \alpha}=-2 \alpha Q_{\mathbb{M}^{d}, \alpha}
$$

and thus,

$$
\left(\mathcal{L}_{\mathbb{M}^{d}, \alpha,+}^{-1} Q_{\mathbb{M}^{d}, \alpha} \mid Q_{\mathbb{M}^{d}, \alpha}\right)_{L^{2}\left(\mathbb{M}^{d}\right)}=-\frac{1}{2 \alpha}\left(\partial_{\alpha} Q_{\mathbb{M}^{d}, \alpha} \mid Q_{\mathbb{M}^{d}, \alpha}\right)_{L^{2}\left(\mathbb{M}^{d}\right)}=-\frac{1}{4 \alpha} \partial_{\alpha}\left\|Q_{\mathbb{M}^{d}, \alpha}\right\|_{L^{2}\left(\mathbb{M}^{d}\right)}^{2} .
$$

Remark 1.9. Theorem 1.7 raises the intriguing question of whether it is possible to "stabilize" the borderline unstable soliton in Euclidean space by changing the background geometry. Unfortunately, we cannot answer this question in the affirmative as we are unable to provide a sufficient criterion for stability in the critical case. This appears to be challenging, as it requires a good understanding of eigenvalues and resonances on the imaginary axis, a question which is still largely open even in the purely Euclidean setting. However, Theorem 1.7 provides a sufficient criterion for (linear) instability. Using this, we provide numerical evidence that there exists a large class of negatively curved manifolds such that the soliton becomes (linearly) unstable, see Section 6. This fits well with the blow-up instability for the $L^{2}$ critical (and super-critical) nonlinear Schrödinger equation on the hyperbolic space $\mathbb{H}^{d}$ computed via virial identities in [4]. Blow-up was also established in [12] in the $L^{2}$ critical setting with a Riemannian manifold that is locally like $\mathbb{H}^{d}$ and asymptotically like $\mathbb{R}^{d}$.

1.2. Further related results. Unfortunately, there is still no general satisfactory understanding of the linearized operator even in the Euclidean case, and as a consequence, this classical problem remains a topic of contemporary research. For instance, see [30] for an analysis of embedded eigenvalues in the essential spectrum and [16] for a modern account of the general theory and new numerical results. Furthermore, decay properties of eigenfunctions are investigated in [42. The paper [59] is concerned with asymptotic stability but also contains a thorough analysis of the linearized operator. In [24], a novel computer-assisted method is introduced to prove the absence of eigenvalues in the essential spectral gap. In addition, in [50], the authors give a numerically assisted proof for the absence of embedded eigenvalues in a variety of settings on $\mathbb{R}^{d}$. In the case of potential perturbations, stability analysis in both the small and large mass limits have been studied through both dispersive 
techniques as well as bifurcation theory on $\mathbb{R}^{d}$ for a range of nonlinearities in many works, for a small sampling see e.g. [69, 27, 39, 45, 44, 49, 54, 53] and the references captured within.

Needless to say, the literature on Schrödinger equations on manifolds is vast and we just mention some closely related recent works. There is a number of papers devoted to the study of the focusing nonlinear Schrödinger equation on hyperbolic space. A recurring theme, compare Remark 1.9, is the question whether the negative curvature may improve the situation compared to the Euclidean case and stabilize the evolution, see, e.g., [2, 1, 4]. Spectral properties in hyperbolic space are studied in [10] and the existence of ground states on noncompact manifolds is investigated in, e.g., [21, 67, 20]. See also the recent works [18, 19, 17] for advances on the spectral measure for asymptotically hyperbolic manifolds, the analysis of which is required for good dispersive estimates that can lead to results on asymptotic stability when understood with perturbations and for the linearized operator. The literature on spectral measures for the asymptotically Euclidean and conic cases is quite vast, but see [48, 11, 40] and references therein.

\section{Preliminary transformations}

We proceed by transforming the radial case of Eq. (1.4) to a standard nonlinear Schrödinger equation on $\mathbb{R}^{d}$ with a potential. This is a well-known reduction, see e.g. [3, 21].

2.1. The Laplace-Beltrami operator. The Laplace-Beltrami operator $\Delta_{\mathbb{M}^{d}}$ is given by

$$
\Delta_{\mathbb{M}^{d}} f=\frac{1}{\sqrt{\operatorname{det} g}} \partial_{j}\left(\sqrt{\operatorname{det} g} g^{j k} \partial_{k} f\right) .
$$

We now assume that $f(r, y)=f(r)$, i.e., we restrict ourselves to the radial case. Then,

$$
\begin{aligned}
\Delta_{\mathbb{M}^{d}} f(r) & =\frac{1}{\sqrt{\operatorname{det} g(r, y)}} \partial_{r}\left(\sqrt{\operatorname{det} g(r, y)} \partial_{r} f(r)\right)=\frac{1}{A(r)^{d-1}} \partial_{r}\left[A(r)^{d-1} \partial_{r} f(r)\right] \\
& =\left[\partial_{r}^{2}+(d-1) \frac{A^{\prime}(r)}{A(r)} \partial_{r}\right] f(r) \\
& =: \Delta_{\mathbb{M}^{d}}^{\mathrm{rad}} f(r) .
\end{aligned}
$$

Obviously, $\Delta_{\mathbb{M}^{d}}^{\mathrm{rad}}$ is formally self-adjoint on $L_{A^{d-1}}^{2}(0, \infty)$. Eq. (1.4) for radial functions reduces to

$$
i \partial_{t} \widetilde{u}(t, \cdot)+\Delta_{\mathbb{M}^{d}}^{\operatorname{rad}} \widetilde{u}(t, \cdot)+\widetilde{u}(t, \cdot)|\widetilde{u}(t, \cdot)|^{p-1}=0
$$

for $\widetilde{u}: \mathbb{R} \times(0, \infty) \rightarrow \mathbb{C}$.

2.2. Conjugation to Euclidean. In order to perturb off the Euclidean case, we would like to compare the Laplace-Beltrami operator $\Delta_{\mathbb{M}^{d}}$ to the ordinary Laplace operator on $\mathbb{R}^{d}$, henceforth denoted by $\Delta_{\mathbb{R}^{d}}$. The restriction of the Euclidean operator to radial functions yields the operator

$$
\Delta_{\mathbb{R}^{d}}^{\mathrm{rad}}:=\partial_{r}^{2}+\frac{d-1}{r} \partial_{r},
$$

acting on $L_{|\cdot| d-1}^{2}(0, \infty)$. To compare the two operators, we need to conjugate by the unitary map that relates the radial function spaces. 
Let $\mathcal{U}_{d}: L_{|\cdot| d-1}^{2}(0, \infty) \rightarrow L_{A^{d-1}}^{2}(0, \infty)$ be defined by

$$
\mathcal{U}_{d} f(r):=\left(\frac{r}{A(r)}\right)^{\frac{d-1}{2}} f(r)
$$

so that $\left\|\mathcal{U}_{d} f\right\|_{L_{A^{d-1}}^{2}(0, \infty)}=\|f\|_{L_{|\cdot| d-1}^{2}(0, \infty)}$. Now we consider the operator $\mathcal{U}_{d}^{-1} \Delta_{\mathbb{M}^{d}}^{\mathrm{rad}} \mathcal{U}_{d}$ on $L_{|\cdot| d-1}^{2}(0, \infty)$. Explicitly, we have

$$
\mathcal{U}_{d}^{-1} \Delta_{\mathbb{M}^{d}}^{\mathrm{rad}} \mathcal{U}_{d} f(r)=\left(\Delta_{\mathbb{R}^{d}}^{\mathrm{rad}}-\frac{d-1}{2} \frac{A^{\prime \prime}(r)}{A(r)}-\frac{(d-1)(d-3)}{4} \frac{A^{\prime}(r)^{2}}{A(r)^{2}}+\frac{(d-1)(d-3)}{4 r^{2}}\right) f(r),
$$

By setting

$$
\widetilde{u}(t, r)=\mathcal{U}_{d}(\widetilde{v}(t, \cdot))(r)=\left(\frac{r}{A(r)}\right)^{\frac{d-1}{2}} \widetilde{v}(t, r),
$$

for a function $\widetilde{v}: \mathbb{R} \times(0, \infty) \rightarrow \mathbb{C}$, Eq. (2.1) can now be written as

$$
i \partial_{t} \widetilde{v}(t, \cdot)+\mathcal{U}_{d}^{-1} \Delta_{\mathbb{M}^{d}}^{\mathrm{rad}} \mathcal{U}_{d} \widetilde{v}(t, \cdot)+\widetilde{v}(t, \cdot)\left|\mathcal{U}_{d} \widetilde{v}(t, \cdot)\right|^{p-1}=0
$$

In fact, we find it more convenient to formulate this equation in terms of the auxiliary function $v: \mathbb{R} \times \mathbb{R}^{d} \rightarrow \mathbb{C}$, given by $v(t, x):=\widetilde{v}(t,|x|)$. This yields

$$
i \partial_{t} v(t, \cdot)+\Delta_{\mathbb{R}^{d}} v(t, \cdot)-V_{d} v(t, \cdot)+\varphi_{d, p} v(t, \cdot)|v(t, \cdot)|^{p-1}=0
$$

with

$$
\varphi_{d, p}(x):=\left(\frac{|x|}{A(|x|)}\right)^{\frac{(d-1)(p-1)}{2}}
$$

and

$$
V_{d}(x):=\frac{d-1}{2} \frac{A^{\prime \prime}(|x|)}{A(|x|)}+\frac{(d-1)(d-3)}{4} \frac{A^{\prime}(|x|)^{2}}{A(|x|)^{2}}-\frac{(d-1)(d-3)}{4|x|^{2}} .
$$

We keep in mind that $v(t, \cdot)$ is radial. Note that Eq. (2.2) resembles a standard nonlinear Schrödinger equation on Euclidean space with a potential $V_{d}$.

To look for solitons, we plug the ansatz $v(t, x)=e^{i \alpha^{2} t} R_{\alpha}(x)$ into Eq. (2.2) with $R_{\alpha}$ radial. This yields the elliptic equation

$$
\Delta_{\mathbb{R}^{d}} R_{\alpha}-\alpha^{2} R_{\alpha}-V_{d} R_{\alpha}+\varphi_{d, p} R_{\alpha}\left|R_{\alpha}\right|^{p-1}=0 .
$$

In terms of the rescaled profile $\widetilde{R}_{\alpha}$, defined by $R_{\alpha}(x)=\alpha^{\frac{2}{p-1}} \widetilde{R}_{\alpha}(\alpha x)$, Eq. (2.3) reads

$$
\Delta_{\mathbb{R}^{d}} \widetilde{R}_{\alpha}(x)-\widetilde{R}_{\alpha}(x)-\alpha^{-2} V_{d}\left(\alpha^{-1} x\right) \widetilde{R}_{\alpha}(x)+\varphi_{d, p}\left(\alpha^{-1} x\right) F_{p}\left(\widetilde{R}_{\alpha}(x)\right)=0,
$$

where $F_{p}(s):=s|s|^{p-1}$. We intend to solve Eq. (2.4) by perturbing off the Euclidean situation and hence insert the ansatz $\widetilde{R}_{\alpha}(x)=Q_{\mathbb{R}^{d}}(x)+\rho(x)$ into Eq. (2.4), where $Q_{\mathbb{R}^{d}}:=Q_{\mathbb{R}^{d}, 1}$. In view of Eq. (1.3), we obtain

$$
\begin{aligned}
-\mathcal{A}_{\alpha} \rho(x)= & q_{\alpha}(x) F_{p}^{\prime}\left(Q_{\mathbb{R}^{d}}(x)\right) \rho(x)+\left[q_{\alpha}(x)-1\right] \mathcal{N}(\rho)(x) \\
& +\alpha^{-2} V_{d}\left(\alpha^{-1} x\right) Q_{\mathbb{R}^{d}}(x)+q_{\alpha}(x) F_{p}\left(Q_{\mathbb{R}^{d}}(x)\right),
\end{aligned}
$$


where

$$
\begin{aligned}
\mathcal{A}_{\alpha} \rho(x) & :=-\Delta_{\mathbb{R}^{d}} \rho(x)+\rho(x)-F_{p}^{\prime}\left(Q_{\mathbb{R}^{d}}(x)\right) \rho(x)+\alpha^{-2} V_{d}\left(\alpha^{-1} x\right) \rho(x), \\
q_{\alpha}(x) & :=1-\varphi_{d, p}\left(\alpha^{-1} x\right), \\
\mathcal{N}(\rho)(x) & :=F_{p}\left(Q_{\mathbb{R}^{d}}(x)+\rho(x)\right)-F_{p}\left(Q_{\mathbb{R}^{d}}(x)\right)-F_{p}^{\prime}\left(Q_{\mathbb{R}^{d}}(x)\right) \rho(x) .
\end{aligned}
$$

\section{Existence of A SOLiton}

In this section we show that Eq. (2.5) has a solution $\rho=\rho_{\alpha}$, provided $\alpha \geq 1$ is sufficiently large. This way, we obtain a soliton solution

$$
v_{\alpha}^{*}(t, x):=\alpha^{\frac{2}{p-1}} e^{i \alpha^{2} t}\left[Q_{\mathbb{R}^{d}}(\alpha x)+\rho_{\alpha}(\alpha x)\right]
$$

to Eq. (2.2).

3.1. Analysis of the linear operator. Initially, we define the operator $\mathcal{A}_{\alpha}$ as a classical differential operator acting on $C_{c}^{\infty}\left(\mathbb{R}^{d}\right)$. Recall that $Q_{\mathbb{R}^{d}} \in C^{\infty}\left(\mathbb{R}^{d}\right), Q_{\mathbb{R}^{d}}>0$, and $V_{d} \in$ $C^{\infty}\left(\mathbb{R}^{d}\right)$ by Hypothesis 1.1. As a consequence, $\mathcal{A}_{\alpha}$ is a continuous map from $\mathcal{D}\left(\mathbb{R}^{d}\right)$ to $\mathcal{D}\left(\mathbb{R}^{d}\right)$. Furthermore, $\mathcal{A}_{\alpha}$ is formally self-adjoint on $L^{2}\left(\mathbb{R}^{d}\right)$ and thus, $\mathcal{A}_{\alpha}$ extends to $\mathcal{D}^{\prime}\left(\mathbb{R}^{d}\right)$ by

$$
\mathcal{A}_{\alpha} u(\varphi):=u\left(\mathcal{A}_{\alpha} \varphi\right)
$$

for $u \in \mathcal{D}^{\prime}\left(\mathbb{R}^{d}\right)$ and $\varphi \in \mathcal{D}\left(\mathbb{R}^{d}\right)$. In the limit $\alpha \rightarrow \infty, \mathcal{A}_{\alpha}$ formally reduces to $\mathcal{L}_{+}$, given by

$$
\mathcal{L}_{+} f(x)=-\Delta_{\mathbb{R}^{d}} f(x)+f(x)-F_{p}^{\prime}\left(Q_{\mathbb{R}^{d}}(x)\right) f(x) .
$$

This is a well-known operator in the Euclidean setting that occurs in the linearization about solitary waves.

Note that both $\mathcal{A}_{\alpha}$ and $\mathcal{L}_{+}$map radial distributions to radial distributions since $Q_{\mathbb{R}^{d}}$ and $V_{d}$ are radial. Consequently, $\mathcal{A}_{\alpha}$ and $\mathcal{L}_{+}$may be viewed as unbounded operators on $L_{\text {rad }}^{2}\left(\mathbb{R}^{d}\right)$.

Lemma 3.1. The operator $\mathcal{L}_{+}: H_{\mathrm{rad}}^{2}\left(\mathbb{R}^{d}\right) \subset L_{\mathrm{rad}}^{2}\left(\mathbb{R}^{d}\right) \rightarrow L_{\mathrm{rad}}^{2}\left(\mathbb{R}^{d}\right)$ is self-adjoint. Furthermore, $\mathcal{L}_{+}$is invertible and we have the smoothing estimate

$$
\left\|\mathcal{L}_{+}^{-1} g\right\|_{H^{2}\left(\mathbb{R}^{d}\right)} \lesssim\|g\|_{L^{2}\left(\mathbb{R}^{d}\right)}
$$

for all $g \in L_{\mathrm{rad}}^{2}\left(\mathbb{R}^{d}\right)$.

Proof. By the exponential decay of $Q_{\mathbb{R}^{d}}$ and [68], p. 258, Theorem 10.2, we see that $\mathcal{L}_{+}$is self-adjoint with domain $H_{\text {rad }}^{2}\left(\mathbb{R}^{d}\right)$ and essential spectrum $\sigma_{e}\left(\mathcal{L}_{+}\right)=[1, \infty)$. Consequently, $0 \notin \sigma\left(\mathcal{L}_{+}\right)$follows from [16], Lemma 2.1. Thus, it remains to prove the smoothing estimate. To this end, let $\mathcal{L}_{0}: H_{\text {rad }}^{2}\left(\mathbb{R}^{d}\right) \subset L_{\text {rad }}^{2}\left(\mathbb{R}^{d}\right) \rightarrow L_{\text {rad }}^{2}\left(\mathbb{R}^{d}\right)$ be given by $\mathcal{L}_{0} f=-\Delta_{\mathbb{R}^{d}} f+f$. For $f \in \mathcal{S}\left(\mathbb{R}^{d}\right)$ we have

$$
\mathcal{F} \mathcal{L}_{0} f(\xi)=\mathcal{F}\left(-\Delta_{\mathbb{R}^{d}} f+f\right)(\xi)=\left(4 \pi^{2}|\xi|^{2}+1\right) \mathcal{F} f(\xi),
$$

where $\mathcal{F}$ denotes the Fourier transform

$$
\mathcal{F} f(\xi):=\int_{\mathbb{R}^{d}} e^{-2 \pi i \xi x} f(x) d x
$$

Thus, on the Fourier side, the equation $\mathcal{L}_{0} f=g$ reads

$$
\left(1+4 \pi^{2}|\xi|^{2}\right) \underset{8}{\mathcal{F}} f(\xi)=\mathcal{F} g(\xi)
$$


Consequently, by Plancherel,

$$
\begin{aligned}
\left\|\mathcal{L}_{0}^{-1} g\right\|_{H^{2}\left(\mathbb{R}^{d}\right)} & =\|f\|_{H^{2}\left(\mathbb{R}^{d}\right)} \simeq\left\|\langle\cdot\rangle^{2} \mathcal{F} f\right\|_{L^{2}\left(\mathbb{R}^{d}\right)}=\left\|\langle\cdot\rangle^{2}\left(1+4 \pi^{2}|\cdot|^{2}\right)^{-1} \mathcal{F} g\right\|_{L^{2}\left(\mathbb{R}^{d}\right)} \lesssim\|\mathcal{F} g\|_{L^{2}\left(\mathbb{R}^{d}\right)} \\
& \simeq\|g\|_{L^{2}\left(\mathbb{R}^{d}\right)}
\end{aligned}
$$

for $g \in \mathcal{S}\left(\mathbb{R}^{d}\right)$. By approximation, this bound holds for all $g \in L_{\mathrm{rad}}^{2}\left(\mathbb{R}^{d}\right)$. Let $\mathcal{B}: L_{\mathrm{rad}}^{2}\left(\mathbb{R}^{d}\right) \rightarrow$ $L_{\text {rad }}^{2}\left(\mathbb{R}^{d}\right)$ be given by $\mathcal{B} f(x)=-F_{p}^{\prime}\left(Q_{\mathbb{R}^{d}}(x)\right) f(x)$. By definition, we have the identity

$$
\mathcal{L}_{+}=\left(1+\mathcal{B L}_{0}^{-1}\right) \mathcal{L}_{0}
$$

and this shows that $\mathcal{L}_{+} \mathcal{L}_{0}^{-1}$ is a bounded operator on $L_{\text {rad }}^{2}\left(\mathbb{R}^{d}\right)$. By the open mapping theorem, its inverse $\mathcal{L}_{0} \mathcal{L}_{+}^{-1}$ is also bounded. Consequently, the smoothing property of $\mathcal{L}_{0}^{-1}$ implies the bound

$$
\left\|\mathcal{L}_{+}^{-1} g\right\|_{H^{2}\left(\mathbb{R}^{d}\right)}=\left\|\mathcal{L}_{0}^{-1} \mathcal{L}_{0} \mathcal{L}_{+}^{-1} g\right\|_{H^{2}\left(\mathbb{R}^{d}\right)} \lesssim\left\|\mathcal{L}_{0} \mathcal{L}_{+}^{-1} g\right\|_{L^{2}\left(\mathbb{R}^{d}\right)} \lesssim\|g\|_{L^{2}\left(\mathbb{R}^{d}\right)}
$$

for all $g \in L_{\mathrm{rad}}^{2}\left(\mathbb{R}^{d}\right)$.

Lemma 3.2. There exists an $\alpha_{0}>0$ such that, for any $\alpha \geq \alpha_{0}$, the operator $\mathcal{A}_{\alpha}: H_{\mathrm{rad}}^{2}\left(\mathbb{R}^{d}\right) \subset$ $L_{\mathrm{rad}}^{2}\left(\mathbb{R}^{d}\right) \rightarrow L_{\mathrm{rad}}^{2}\left(\mathbb{R}^{d}\right)$ is self-adjoint and invertible. Furthermore, we have the smoothing estimate

$$
\left\|\mathcal{A}_{\alpha}^{-1} f\right\|_{H^{2}\left(\mathbb{R}^{d}\right)} \lesssim\|f\|_{L^{2}\left(\mathbb{R}^{d}\right)}
$$

for all $f \in L_{\mathrm{rad}}^{2}\left(\mathbb{R}^{d}\right)$ and all $\alpha \geq \alpha_{0}$.

Proof. For any $\alpha>0$ we define a bounded operator $\mathcal{B}_{\alpha}$ on $L_{\text {rad }}^{2}\left(\mathbb{R}^{d}\right)$ by setting

$$
\mathcal{B}_{\alpha} f(x):=-V_{d}\left(\alpha^{-1} x\right) f(x) .
$$

Since $V_{d} \in L^{\infty}\left(\mathbb{R}^{d}\right)$ by Hypothesis 1.1, we infer $\left\|\mathcal{B}_{\alpha} f\right\|_{L^{2}\left(\mathbb{R}^{d}\right)} \lesssim\|f\|_{L^{2}\left(\mathbb{R}^{d}\right)}$ for all $\alpha>0$. Consequently, a Neumann series argument shows the existence of the operator $\left(1-\alpha^{-2} \mathcal{B}_{\alpha} \mathcal{L}_{+}^{-1}\right)^{-1}$ with the bound

$$
\left\|\left(1-\alpha^{-2} \mathcal{B}_{\alpha} \mathcal{L}_{+}^{-1}\right)^{-1} f\right\|_{L^{2}\left(\mathbb{R}^{d}\right)} \lesssim\|f\|_{L^{2}\left(\mathbb{R}^{d}\right)}
$$

for all $\alpha \geq \alpha_{0}$, provided $\alpha_{0}>0$ is sufficiently large. Thus, from the identity $\mathcal{A}_{\alpha}=(1-$ $\left.\alpha^{-2} \mathcal{B}_{\alpha} \mathcal{L}_{+}^{-1}\right) \mathcal{L}_{+}$, we obtain the existence of the operator

$$
\mathcal{A}_{\alpha}^{-1}=\mathcal{L}_{+}^{-1}\left(1-\alpha^{-2} \mathcal{B}_{\alpha} \mathcal{L}_{+}^{-1}\right)^{-1}
$$

with the bound

$$
\begin{aligned}
\left\|\mathcal{A}_{\alpha}^{-1} f\right\|_{H^{2}\left(\mathbb{R}^{d}\right)} & \lesssim\left\|\mathcal{L}_{+}^{-1}\left(1-\alpha^{-2} \mathcal{B}_{\alpha} \mathcal{L}_{+}^{-1}\right)^{-1} f\right\|_{H^{2}\left(\mathbb{R}^{d}\right)} \lesssim\left\|\left(1-\alpha^{-2} \mathcal{B}_{\alpha} \mathcal{L}_{+}^{-1}\right)^{-1} f\right\|_{L^{2}\left(\mathbb{R}^{d}\right)} \\
& \lesssim\|f\|_{L^{2}\left(\mathbb{R}^{d}\right)}
\end{aligned}
$$

for all $f \in L_{\text {rad }}^{2}\left(\mathbb{R}^{d}\right)$ and $\alpha \geq \alpha_{0}$.

As a consequence of Lemma 3.2. we can now reformulate Eq. (2.5) as the fixed point problem

$$
\rho=-\mathcal{A}_{\alpha}^{-1}\left[q_{\alpha} F_{p}^{\prime}\left(Q_{\mathbb{R}^{d}}(\cdot)\right) \rho+\left(q_{\alpha}-1\right) \mathcal{N}(\rho)+\alpha^{-2} V_{d}\left(\alpha^{-1}(\cdot)\right) Q_{\mathbb{R}^{d}}+q_{\alpha} F_{p}\left(Q_{\mathbb{R}^{d}}(\cdot)\right)\right] .
$$


3.2. Refined bounds for $\mathcal{A}_{\alpha}^{-1}$. Next, we prove an $L^{\infty}$ bound for $\mathcal{A}_{\alpha}^{-1}$, again by first proving the corresponding result for $\mathcal{L}_{+}^{-1}$.

Lemma 3.3. We have the bound

$$
\left\|\mathcal{L}_{+}^{-1} g\right\|_{L^{\infty}\left(\mathbb{R}^{d}\right)} \lesssim\|g\|_{L^{2}\left(\mathbb{R}^{d}\right)}+\|g\|_{L^{\infty}\left(\mathbb{R}^{d}\right)}
$$

for all $g \in L_{\mathrm{rad}}^{2}\left(\mathbb{R}^{d}\right) \cap L^{\infty}\left(\mathbb{R}^{d}\right) \cap C\left(\mathbb{R}^{d}\right)$.

Proof. By Sobolev embedding and Lemma 3.1 the result is immediate in the case $d=2$, i.e.,

$$
\left\|\mathcal{L}_{+}^{-1} g\right\|_{L^{\infty}\left(\mathbb{R}^{2}\right)} \lesssim\left\|\mathcal{L}_{+}^{-1} g\right\|_{H^{2}\left(\mathbb{R}^{2}\right)} \lesssim\|g\|_{L^{2}\left(\mathbb{R}^{2}\right)}
$$

Thus, we may restrict ourselves to $d \geq 3$. Since all functions are radial, problems occur only at the origin. Indeed, by the one-dimensional Sobolev embedding and Lemma 3.1, we have

$$
\left\|\mathcal{L}_{+}^{-1} g\right\|_{L^{\infty}\left(\mathbb{R}^{d} \backslash \mathbb{B}^{d}\right)} \lesssim\left\|\mathcal{L}_{+}^{-1} g\right\|_{H^{1}\left(\mathbb{R}^{d}\right)} \lesssim\|g\|_{L^{2}\left(\mathbb{R}^{d}\right)}
$$

for all $g \in L_{\mathrm{rad}}^{2}\left(\mathbb{R}^{d}\right)$. Consequently, it suffices to prove the estimate

$$
\left\|\mathcal{L}_{+}^{-1} g\right\|_{L^{\infty}\left(\mathbb{B}^{d}\right)} \lesssim\|g\|_{L^{2}\left(\mathbb{R}^{d}\right)}+\|g\|_{L^{\infty}\left(\mathbb{R}^{d}\right)}
$$

for all $g \in L_{\mathrm{rad}}^{2}\left(\mathbb{R}^{d}\right) \cap L^{\infty}\left(\mathbb{R}^{d}\right) \cap C\left(\mathbb{R}^{d}\right)$.

Let $f=\mathcal{L}_{+}^{-1} g$. Then $f \in H_{\text {rad }}^{2}\left(\mathbb{R}^{d}\right)$, and by the radial Sobolev embedding we infer that $f \in C\left(\mathbb{R}^{d} \backslash\{0\}\right) \cap L_{\text {loc }}^{1}\left(\mathbb{R}^{d}\right)$. The equation $\mathcal{L}_{+} f=g$ implies $\Delta_{\mathbb{R}^{d}} f^{\sharp}=h^{\sharp}$ in $\mathcal{D}^{\prime}\left(\mathbb{R}^{d}\right)$ with

$$
h(x):=-g(x)+f(x)-F_{p}^{\prime}\left(Q_{\mathbb{R}^{d}}(x)\right) f(x),
$$

see Definition $\mathrm{A.2}$ for the notation. Evidently, $h \in C\left(\mathbb{R}^{d} \backslash\{0\}\right) \cap L_{\text {loc }}^{1}\left(\mathbb{R}^{d}\right)$ and thus, Lemma A.3 shows that the function $\widehat{f}(r):=r^{\frac{d-1}{2}} f\left(r e_{1}\right)$ belongs to $C^{2}(0, \infty)$ and satisfies

$$
\widehat{f}^{\prime \prime}(r)-\frac{(d-1)(d-3)}{4 r^{2}} \widehat{f}(r)-\widehat{f}(r)+F_{p}^{\prime}\left(Q_{\mathbb{R}^{d}}\left(r e_{1}\right)\right) \widehat{f}(r)=-r^{\frac{d-1}{2}} g\left(r e_{1}\right)
$$

for all $r>0$. Now we consider the homogeneous version of Eq. (3.2), i.e.,

$$
\phi^{\prime \prime}(r)-\frac{(d-1)(d-3)}{4 r^{2}} \phi(r)-\phi(r)+F_{p}^{\prime}\left(Q_{\mathbb{R}^{d}}\left(r e_{1}\right)\right) \phi(r)=0 .
$$

Eq. (3.3) has a fundamental system $\left\{\phi_{0}, \phi_{\infty}\right\}$ with the asymptotic behavior

$$
\begin{array}{rlrl}
\left|\phi_{0}(r)\right| & \simeq r^{\frac{d-1}{2}} \text { for } r \in[0,1], & & \left|\phi_{0}(r)\right| \simeq e^{r} \text { for } r \geq 1, \\
\left|\phi_{0}^{\prime}(r)\right| \simeq r^{\frac{d-3}{2}} \text { for } r \in[0,1], & & \left|\phi_{0}^{\prime}(r)\right| \simeq e^{r} \text { for } r \geq 1, \\
\left|\phi_{\infty}(r)\right| \simeq r^{-\frac{d-3}{2}} \text { for } r \in(0,1], & & \left|\phi_{\infty}(r)\right| \simeq e^{-r} \text { for } r \geq 1, \\
\left|\phi_{\infty}^{\prime}(r)\right| \simeq r^{-\frac{d-1}{2}} \text { for } r \in(0,1], & & \left|\phi_{\infty}^{\prime}(r)\right| \simeq e^{-r} \text { for } r \geq 1,
\end{array}
$$

and we may normalize so that $W\left(\phi_{0}, \phi_{\infty}\right)=1$, see Lemma B.1 below. Consequently, the variation of constants formula yields the existence of constants $a, b \in \mathbb{C}$ such that

$$
\widehat{f}(r)=a \phi_{0}(r)+b \phi_{\infty}(r)-\phi_{0}(r) \int_{r}^{\infty} \phi_{\infty}(s) s^{\frac{d-1}{2}} g\left(s e_{1}\right) d s-\phi_{\infty}(r) \int_{0}^{r} \phi_{0}(s) s^{\frac{d-1}{2}} g\left(s e_{1}\right) d s,
$$


and $f \in H_{\mathrm{rad}}^{1}\left(\mathbb{R}^{d}\right)$ implies that $a=b=0$. Furthermore, we have the bounds

$$
\begin{aligned}
\left|\phi_{0}(r)\right| \int_{r}^{\infty}\left|\phi_{\infty}(s) s^{\frac{d-1}{2}} g\left(s e_{1}\right)\right| d s & \lesssim r^{\frac{d-1}{2}}\|g\|_{L^{\infty}\left(\mathbb{R}^{d}\right)}\left[\int_{0}^{1} s^{-\frac{d-3}{2}} s^{\frac{d-1}{2}} d s+\int_{1}^{\infty} e^{-s} s^{\frac{d-1}{2}} d s\right] \\
& \lesssim r^{\frac{d-1}{2}}\|g\|_{L^{\infty}\left(\mathbb{R}^{d}\right)}
\end{aligned}
$$

and

$$
\begin{aligned}
\left|\phi_{\infty}(r)\right| \int_{0}^{r}\left|\phi_{0}(s) s^{\frac{d-1}{2}} g\left(s e_{1}\right)\right| d s & \lesssim r^{-\frac{d-3}{2}}\|g\|_{L^{\infty}\left(\mathbb{R}^{d}\right)} \int_{0}^{r} s^{d-1} d s \\
& \lesssim r^{\frac{d+3}{2}}\|g\|_{L^{\infty}\left(\mathbb{R}^{d}\right)}
\end{aligned}
$$

for all $r \in(0,1]$. Consequently,

$$
\left|f\left(r e_{1}\right)\right|=\left|r^{-\frac{d-1}{2}} \widehat{f}(r)\right| \lesssim\|g\|_{L^{\infty}\left(\mathbb{R}^{d}\right)}
$$

for all $r \in(0,1]$, which implies the desired bound

$$
\left\|\mathcal{L}_{+}^{-1} g\right\|_{L^{\infty}\left(\mathbb{B}^{d}\right)}=\|f\|_{L^{\infty}\left(\mathbb{B}^{d}\right)} \lesssim\|g\|_{L^{\infty}\left(\mathbb{R}^{d}\right)} .
$$

By a simple perturbative argument, we obtain an analogous $L^{\infty}$ bound for the operator $\mathcal{A}_{\alpha}^{-1}$.

Corollary 3.4. There exists an $\alpha_{0}>0$ such that

$$
\left\|\mathcal{A}_{\alpha}^{-1} g\right\|_{L^{\infty}\left(\mathbb{R}^{d}\right)} \lesssim\|g\|_{L^{2}\left(\mathbb{R}^{d}\right)}+\|g\|_{L^{\infty}\left(\mathbb{R}^{d}\right)}
$$

for all $g \in L_{\text {rad }}^{2}\left(\mathbb{R}^{d}\right) \cap L^{\infty}\left(\mathbb{R}^{d}\right) \cap C\left(\mathbb{R}^{d}\right)$ and $\alpha \geq \alpha_{0}$.

Proof. Let $X:=L_{\text {rad }}^{2}\left(\mathbb{R}^{d}\right) \cap L^{\infty}\left(\mathbb{R}^{d}\right) \cap C\left(\mathbb{R}^{d}\right)$ and write

$$
\|g\|_{X}:=\|g\|_{L^{2}\left(\mathbb{R}^{d}\right)}+\|g\|_{L^{\infty}\left(\mathbb{R}^{d}\right)} .
$$

As in the proof of Lemma 3.2, we set

$$
\mathcal{B}_{\alpha} g(x):=-V_{d}\left(\alpha^{-1} x\right) g(x) .
$$

Note that $\left\|\mathcal{B}_{\alpha} g\right\|_{X} \lesssim\|g\|_{X}$ for all $g \in X$ and $\alpha>0$ by Hypothesis 1.1. Consequently, the operator $\left(1-\alpha^{-2} \mathcal{B}_{\alpha}\right): X \rightarrow X$ is bounded invertible on $X$ for any $\alpha \geq \alpha_{0}$ by a Neumann series argument, provided $\alpha_{0}$ is sufficiently large. Furthermore,

$$
\left\|\left(1-\alpha^{-2} \mathcal{B}_{\alpha}\right)^{-1} g\right\|_{X} \lesssim\|g\|_{X}
$$

for all $g \in X$ and $\alpha \geq \alpha_{0}$. By Lemmas 3.1 and 3.3, we have $\left\|\mathcal{L}_{+}^{-1} g\right\|_{X} \lesssim\|g\|_{X}$ for all $g \in X$, and thus,

$$
\begin{aligned}
\left\|\mathcal{A}_{\alpha}^{-1} g\right\|_{L^{\infty}\left(\mathbb{R}^{d}\right)} & \leq\left\|\mathcal{A}_{\alpha}^{-1} g\right\|_{X}=\left\|\mathcal{L}_{+}^{-1}\left(1-\alpha^{-2} \mathcal{B}_{\alpha} \mathcal{L}_{+}^{-1}\right)^{-1} g\right\|_{X} \lesssim\left\|\left(1-\alpha^{-1} \mathcal{B}_{\alpha} \mathcal{L}_{+}^{-1}\right)^{-1} g\right\|_{X} \lesssim\|g\|_{X} \\
& \lesssim\|g\|_{L^{2}\left(\mathbb{R}^{d}\right)}+\|g\|_{L^{\infty}\left(\mathbb{R}^{d}\right)}
\end{aligned}
$$

for all $g \in X$ and $\alpha \geq \alpha_{0}$, as desired. 
3.3. Bounds on the right-hand side of Eq. (3.1). Next, we provide suitable estimates for the terms appearing on the right-hand side of Eq. (3.1).

Lemma 3.5. We have the bounds

$$
\begin{aligned}
\left|q_{\alpha}(x)\right| & \lesssim 1, \\
\left|q_{\alpha}(x) F_{p}^{\prime}\left(Q_{\mathbb{R}^{d}}(x)\right)\right| & \lesssim \alpha^{-1}, \\
\left|\alpha^{-2} V_{d}\left(\alpha^{-1} x\right) Q_{\mathbb{R}^{d}}(x)\right| & \lesssim \alpha^{-2}\langle x\rangle^{-d}, \\
\left|q_{\alpha}(x) F_{p}\left(Q_{\mathbb{R}^{d}}(x)\right)\right| & \lesssim \alpha^{-1}\langle x\rangle^{-d},
\end{aligned}
$$

for all $x \in \mathbb{R}^{d}$ and all $\alpha \geq 1$.

Proof. Recall that we assume Hypothesis 1.1. For $r \in\left[0, \alpha^{\frac{1}{2}}\right]$ we have

$$
\frac{\alpha^{-1} r}{A\left(\alpha^{-1} r\right)}=\frac{\alpha^{-1} r}{\alpha^{-1} r\left[1+O\left(\alpha^{-2} r^{2}\right)\right]}=1+O\left(\alpha^{-1}\right),
$$

and thus,

$$
\left|q_{\alpha}(x)\right|=\left|1-\varphi_{d, p}\left(\alpha^{-1} x\right)\right|=\left|1-\left(\frac{\left|\alpha^{-1} x\right|}{A\left(\left|\alpha^{-1} x\right|\right)}\right)^{\frac{(d-1)(p-1)}{2}}\right| \lesssim \alpha^{-1}
$$

for all $|x| \leq \alpha^{\frac{1}{2}}$. For $|x| \geq \alpha^{\frac{1}{2}}$ we trivially estimate

$$
\left|q_{\alpha}(x)\right| \lesssim 1+\left(\frac{\left|\alpha^{-1} x\right|}{A\left(\left|\alpha^{-1} x\right|\right)}\right)^{\frac{(d-1)(p-1)}{2}} \lesssim 1
$$

since $A\left(\left|\alpha^{-1} x\right|\right) \gtrsim\left|\alpha^{-1} x\right|$. This yields the first statement.

For the second one we recall that $F_{p}^{\prime}\left(Q_{\mathbb{R}^{d}}(x)\right)=p\left|Q_{\mathbb{R}^{d}}(x)\right|^{p-1}$ decays exponentially as $|x| \rightarrow \infty$. In particular, $\left|F_{p}^{\prime}\left(Q_{\mathbb{R}^{d}}(x)\right)\right| \lesssim\langle x\rangle^{-2}$ and thus,

$$
\left|q_{\alpha}(x) F_{p}^{\prime}\left(Q_{\mathbb{R}^{d}}(x)\right)\right| \lesssim\left\langle\alpha^{\frac{1}{2}}\right\rangle^{-2} \lesssim \alpha^{-1}
$$

provided $|x| \geq \alpha^{\frac{1}{2}}$. In the case $|x| \leq \alpha^{\frac{1}{2}}$ we use the bound $\left|q_{\alpha}(x)\right| \lesssim \alpha^{-1}$ from above. This proves the second bound, and the fourth bound follows analogously. Finally, the third estimate is obvious from $V_{d} \in L^{\infty}\left(\mathbb{R}^{d}\right)$ and the exponential decay of $Q_{\mathbb{R}^{d}}$.

Next, we provide Lipschitz estimates for the nonlinearity from Eq. (3.1).

Lemma 3.6. We have the bound

$$
\|\mathcal{N}(f)-\mathcal{N}(g)\|_{L^{2}\left(\mathbb{R}^{d}\right)} \lesssim\left(\|f\|_{H^{2}\left(\mathbb{R}^{d}\right)}^{p-1}+\|f\|_{H^{2}\left(\mathbb{R}^{d}\right)}+\|g\|_{H^{2}\left(\mathbb{R}^{d}\right)}^{p-1}+\|g\|_{H^{2}\left(\mathbb{R}^{d}\right)}\right)\|f-g\|_{H^{2}\left(\mathbb{R}^{d}\right)}
$$

for all $f, g \in H^{2}\left(\mathbb{R}^{d}\right)$. Furthermore,

$$
\|\mathcal{N}(f)-\mathcal{N}(g)\|_{L^{\infty}\left(\mathbb{R}^{d}\right)} \lesssim\left(\|f\|_{L^{\infty}\left(\mathbb{R}^{d}\right)}^{p-1}+\|f\|_{L^{\infty}\left(\mathbb{R}^{d}\right)}+\|g\|_{L^{\infty}\left(\mathbb{R}^{d}\right)}^{p-1}+\|g\|_{L^{\infty}\left(\mathbb{R}^{d}\right)}\right)\|f-g\|_{L^{\infty}\left(\mathbb{R}^{d}\right)}
$$

for all $f, g \in L^{\infty}\left(\mathbb{R}^{d}\right)$.

Proof. Recall that we assume $p \in\left(1, \frac{d+2}{d-2}\right)$ and $d \geq 2$. Let $N\left(t_{0}, t\right):=F_{p}\left(t_{0}+t\right)-F_{p}\left(t_{0}\right)-$ $F_{p}^{\prime}\left(t_{0}\right) t$. Then we have

$$
\mathcal{N}(\rho)(x)=N\left(Q_{\mathbb{R}^{d}}(x), \rho(x)\right),
$$


and the fundamental theorem of calculus yields

$$
\begin{aligned}
N\left(t_{0}, t\right)-N\left(t_{0}, s\right) & =\int_{0}^{1} \partial_{u} N\left(t_{0}, s+u(t-s)\right) d u \\
& =(t-s) \int_{0}^{1}\left[F_{p}^{\prime}\left(t_{0}+s+u(t-s)\right)-F_{p}^{\prime}\left(t_{0}\right)\right] d u .
\end{aligned}
$$

Now we distinguish the cases $p \in(1,2]$ and $p>2$. We proceed with the former and note the elementary estimate

$$
|| t_{0}+\left.t\right|^{p-1}-\left.\left|t_{0}\right|^{p-1}|\lesssim| t\right|^{p-1}
$$

for all $t_{0}, t \in \mathbb{R}$. Since $F_{p}^{\prime}(s)=p|s|^{p-1}$, we obtain from Eq. (3.4) the bound

$$
\begin{aligned}
\left|N\left(t_{0}, t\right)-N\left(t_{0}, s\right)\right| & \lesssim|t-s| \int_{0}^{1}|s+u(t-s)|^{p-1} d u \\
& \lesssim\left(|t|^{p-1}+|s|^{p-1}\right)|t-s|
\end{aligned}
$$

for all $t_{0}, t, s \in \mathbb{R}$. Consequently, by Hölder's inequality and Sobolev embedding,

$$
\begin{aligned}
\|\mathcal{N}(f)-\mathcal{N}(g)\|_{L^{2}\left(\mathbb{R}^{d}\right)} & \lesssim\left\||f|^{p-1}(f-g)\right\|_{L^{2}\left(\mathbb{R}^{d}\right)}+\left\||g|^{p-1}(f-g)\right\|_{L^{2}\left(\mathbb{R}^{d}\right)} \\
& \lesssim\|f\|_{L^{2 p}\left(\mathbb{R}^{d}\right)}^{p-1}\|f-g\|_{L^{2 p}\left(\mathbb{R}^{d}\right)}+\|g\|_{L^{2 p}\left(\mathbb{R}^{d}\right)}^{p-1}\|f-g\|_{L^{2 p}\left(\mathbb{R}^{d}\right)} \\
& \lesssim\left(\|f\|_{H^{2}\left(\mathbb{R}^{d}\right)}^{p-1}+\|g\|_{H^{2}\left(\mathbb{R}^{d}\right)}^{p-1}\right)\|f-g\|_{H^{2}\left(\mathbb{R}^{d}\right)} .
\end{aligned}
$$

In the case $p>2$ (which only occurs if $d \leq 5$ ), we use the bound

$$
|| t_{0}+\left.t\right|^{p-1}-\left.\left|t_{0}\right|^{p-1}|\lesssim| t\right|^{p-1}+\left|t_{0}\right|^{p-2}|t|
$$

which yields

$$
\begin{aligned}
\left|N\left(t_{0}, t\right)-N\left(t_{0}, s\right)\right| & \lesssim|t-s| \int_{0}^{1}\left(|s+u(t-s)|^{p-1}+\left|t_{0}\right|^{p-2}|s+u(t-s)|\right) d u \\
& \lesssim\left(|t|^{p-1}+\left|t_{0}\right|^{p-2}|t|+|s|^{p-1}+\left|t_{0}\right|^{p-2}|s|\right)|t-s|
\end{aligned}
$$

for all $t_{0}, t, s \in \mathbb{R}$. Consequently,

$$
\begin{aligned}
\|\mathcal{N}(f)-\mathcal{N}(g)\|_{L^{2}\left(\mathbb{R}^{d}\right)} \lesssim & \left\|\left.f\right|^{p-1}(f-g)\right\|_{L^{2}\left(\mathbb{R}^{d}\right)}+\left\|Q_{\mathbb{R}^{d}}\right\|_{L^{\infty}\left(\mathbb{R}^{d}\right)}^{p-2}\|f(f-g)\|_{L^{2}\left(\mathbb{R}^{d}\right)} \\
& +\left\||g|^{p-1}(f-g)\right\|_{L^{2}\left(\mathbb{R}^{d}\right)}+\left\|Q_{\mathbb{R}^{d}}\right\|_{L^{\infty}\left(\mathbb{R}^{d}\right)}^{p-2}\|g(f-g)\|_{L^{2}\left(\mathbb{R}^{d}\right)} \\
\lesssim & \|f\|_{L^{2 p\left(\mathbb{R}^{d}\right)}}^{p-1}\|f-g\|_{L^{2 p}\left(\mathbb{R}^{d}\right)}+\|f\|_{L^{4}\left(\mathbb{R}^{d}\right)}\|f-g\|_{L^{4}\left(\mathbb{R}^{d}\right)} \\
& +\|g\|_{L^{2 p}\left(\mathbb{R}^{d}\right)}^{p-1}\|f-g\|_{L^{2 p\left(\mathbb{R}^{d}\right)}}+\|g\|_{L^{4}\left(\mathbb{R}^{d}\right)}\|f-g\|_{L^{4}\left(\mathbb{R}^{d}\right)} \\
\lesssim & \left(\|f\|_{H^{2}\left(\mathbb{R}^{d}\right)}^{p-1}+\|f\|_{H^{2}\left(\mathbb{R}^{d}\right)}+\|g\|_{H^{2}\left(\mathbb{R}^{d}\right)}^{p-1}+\|g\|_{H^{2}\left(\mathbb{R}^{d}\right)}\right)\|f-g\|_{H^{2}\left(\mathbb{R}^{d}\right)},
\end{aligned}
$$

by the Sobolev embeddings $H^{2}\left(\mathbb{R}^{d}\right) \hookrightarrow L^{2 p}\left(\mathbb{R}^{d}\right)$ and $H^{2}\left(\mathbb{R}^{d}\right) \hookrightarrow L^{4}\left(\mathbb{R}^{d}\right)$ (recall that $\left.d \leq 5\right)$. The $L^{\infty}$ bound is immediate from the above. 
3.4. Existence of the soliton. Now we are ready to prove the existence of the soliton profile $Q_{\mathbb{M}^{d}, \alpha}$.

Proposition 3.7. There exists an $\alpha_{0}>0$ such that Eq. (3.1) has a real-valued solution $\rho=\rho_{\alpha} \in H_{\text {rad }}^{2}\left(\mathbb{R}^{d}\right) \cap C\left(\mathbb{R}^{d}\right)$ for any $\alpha \geq \alpha_{0}$. Furthermore, $\rho_{\alpha}$ satisfies

$$
\left\|\rho_{\alpha}\right\|_{H^{2}\left(\mathbb{R}^{d}\right)}+\left\|\rho_{\alpha}\right\|_{L^{\infty}\left(\mathbb{R}^{d}\right)} \lesssim \alpha^{-1}
$$

for all $\alpha \geq \alpha_{0}$.

Proof. Let $X:=H_{\mathrm{rad}}^{2}\left(\mathbb{R}^{d}\right) \cap C\left(\mathbb{R}^{d}\right)$ with norm

$$
\|f\|_{X}:=\|f\|_{H^{2}\left(\mathbb{R}^{d}\right)}+\|f\|_{L^{\infty}\left(\mathbb{R}^{d}\right)}
$$

and set $X_{\delta}:=\left\{f \in X:\|f\|_{X} \leq \delta\right\}$. Similarly, we define $Y:=L_{\text {rad }}^{2}\left(\mathbb{R}^{d}\right) \cap L^{\infty}\left(\mathbb{R}^{d}\right)$ with

$$
\|f\|_{Y}:=\|f\|_{L^{2}\left(\mathbb{R}^{d}\right)}+\|f\|_{L^{\infty}\left(\mathbb{R}^{d}\right)} .
$$

Note that Lemmas [3.2, 3.6 and Corollary 3.4 imply the estimates

$$
\begin{aligned}
\left\|\mathcal{A}_{\alpha}^{-1} f\right\|_{X} & \lesssim\|f\|_{Y} \\
\|\mathcal{N}(f)-\mathcal{N}(g)\|_{Y} & \lesssim\left(\|f\|_{X}+\|f\|_{X}^{p-1}+\|g\|_{X}+\|g\|_{X}^{p-1}\right)\|f-g\|_{X}
\end{aligned}
$$

for all $f, g \in X \subset Y$ and $\alpha \geq \alpha_{0}$, provided $\alpha_{0}>0$ is sufficiently large. In view of Eq. (3.1), we define a map $\mathcal{K}_{\alpha}$ on $X_{\delta}$ by

$$
\mathcal{K}_{\alpha}(f):=-\mathcal{A}_{\alpha}^{-1}\left[q_{\alpha} F_{p}^{\prime}\left(Q_{\mathbb{R}^{d}}(\cdot)\right) f+\left(q_{\alpha}-1\right) \mathcal{N}(f)+\alpha^{-2} V_{d}\left(\alpha^{-1}(\cdot)\right) Q_{\mathbb{R}^{d}}+q_{\alpha} F_{p}\left(Q_{\mathbb{R}^{d}}(\cdot)\right)\right] .
$$

Then, by Lemma 3.5 ,

$$
\begin{aligned}
\left\|\mathcal{K}_{\alpha}(f)\right\|_{X} \lesssim & \left\|q_{\alpha} F_{p}^{\prime}\left(Q_{\mathbb{R}^{d}}(\cdot)\right) f\right\|_{Y}+\left\|\left(q_{\alpha}-1\right) \mathcal{N}(f)\right\|_{Y} \\
& +\alpha^{-2}\left\|V_{d}\left(\alpha^{-1}(\cdot)\right) Q_{\mathbb{R}^{d}}\right\|_{Y}+\left\|q_{\alpha} F_{p}\left(Q_{\mathbb{R}^{d}}(\cdot)\right)\right\|_{Y} \\
\lesssim & \alpha^{-1}\|f\|_{X}+\|f\|_{X}^{p}+\|f\|_{X}^{2}+\alpha^{-2}+\alpha^{-1} \\
\lesssim & \alpha^{-1} \delta+\delta^{p}+\delta^{2}+\alpha^{-2}+\alpha^{-1}
\end{aligned}
$$

for all $f \in X_{\delta}$. Thus, $\mathcal{K}_{\alpha}(f) \in X_{\delta}$ for all $f \in X_{\delta}$ and $\alpha \geq \alpha_{0}$, provided $\delta>0$ is sufficiently small and $\alpha_{0} \geq 1$ is sufficiently large. Similarly,

$$
\begin{aligned}
& \left\|\mathcal{K}_{\alpha}(f)-\mathcal{K}_{\alpha}(g)\right\|_{X} \\
& \quad \lesssim\left\|q_{\alpha} F_{p}^{\prime}\left(Q_{\mathbb{R}^{d}}(\cdot)\right)(f-g)\right\|_{Y}+\left\|\left(q_{\alpha}-1\right)(\mathcal{N}(f)-\mathcal{N}(g))\right\|_{Y} \\
& \quad \lesssim \alpha^{-1}\|f-g\|_{X}+\left(\|f\|_{X}^{p-1}+\|f\|_{X}+\|g\|_{X}^{p-1}+\|g\|_{X}\right)\|f-g\|_{X} \\
& \quad \lesssim\left(\alpha^{-1}+\delta^{p-1}+\delta\right)\|f-g\|_{X} .
\end{aligned}
$$

Thus, $\mathcal{K}_{\alpha}$ is a contraction on $X_{\delta}$ for all $\alpha \geq \alpha_{0}$, provided $\delta>0$ is small enough and $\alpha_{0} \geq 1$ is large enough. Consequently, the contraction mapping principle yields the existence of a fixed point $\rho_{\alpha} \in X_{\delta} \subset H_{\text {rad }}^{2}\left(\mathbb{R}^{d}\right) \cap C\left(\mathbb{R}^{d}\right)$ of $\mathcal{K}_{\alpha}$ which, by construction, is a solution to Eq. (3.1). Finally, for the stated estimate on $\rho_{\alpha}$, it suffices to note that

$$
\left\|\rho_{\alpha}\right\|_{X}=\left\|\mathcal{K}_{\alpha}\left(\rho_{\alpha}\right)\right\|_{X} \lesssim \alpha^{-1}\left\|\rho_{\alpha}\right\|_{X}+\delta^{p-1}\left\|\rho_{\alpha}\right\|_{X}+\delta\left\|\rho_{\alpha}\right\|_{X}+\alpha^{-2}+\alpha^{-1},
$$

by the above estimate for $\mathcal{K}_{\alpha}(f)$. 
3.5. Decay and regularity. From now on we denote by $\rho_{\alpha}$ the solution constructed in Proposition 3.7. Note that the radiality of $\rho_{\alpha}$ immediately implies a pointwise decay estimate. To see this, we recall the classical Strauss estimate.

Lemma 3.8. We have the bound

$$
\left\||\cdot| \frac{d-1}{2} f\right\|_{L^{\infty}\left(\mathbb{R}^{d}\right)} \lesssim\|f\|_{H^{1}\left(\mathbb{R}^{d}\right)}
$$

for all $f \in H_{\mathrm{rad}}^{1}\left(\mathbb{R}^{d}\right)$.

Proof. It suffices to prove the bound for real-valued $f$. First, we assume that $f \in C_{c}^{\infty}\left(\mathbb{R}^{d}\right)$. Then $f$ is given by $f(x)=\widehat{f}(|x|)$ for some $\widehat{f} \in C_{c}^{\infty}(\mathbb{R})$. By the fundamental theorem of calculus and Cauchy-Schwarz, we obtain

$$
\begin{aligned}
r^{d-1} \widehat{f}(r)^{2} & =-\int_{r}^{\infty} \partial_{s}\left[s^{d-1} \widehat{f}(s)^{2}\right] d s=-(d-1) \int_{r}^{\infty} s^{d-2} \widehat{f}(s)^{2} d s-2 \int_{r}^{\infty} s^{d-1} \widehat{f^{\prime}}(s) \widehat{f}(s) d s \\
& \lesssim\|f\|_{L^{2}\left(\mathbb{R}^{d}\right)}\|\nabla f\|_{L^{2}\left(\mathbb{R}^{d}\right)} \lesssim\|f\|_{H^{1}\left(\mathbb{R}^{d}\right)}^{2}
\end{aligned}
$$

for all $r \geq 0$, which implies the desired estimate. By approximation, the bound extends to all $f \in \overline{H_{\text {rad }}^{1}}\left(\mathbb{R}^{d}\right)$.

Lemma 3.8 implies the decay

$$
\left|\rho_{\alpha}(x)\right| \lesssim\langle x\rangle^{-\frac{d-1}{2}}
$$

for all $x \in \mathbb{R}^{d}$.

Lemma 3.9. We have $\rho_{\alpha} \in C^{2}\left(\mathbb{R}^{d}\right)$. In particular, the function $\widetilde{R}_{\alpha}(x)=Q_{\mathbb{R}^{d}}(x)+\rho_{\alpha}(x)$ satisfies

$$
\Delta_{\mathbb{R}^{d}} \widetilde{R}_{\alpha}(x)-\widetilde{R}_{\alpha}(x)-\alpha^{-2} V_{d}\left(\alpha^{-1} x\right) \widetilde{R}_{\alpha}(x)+\varphi_{d, p}\left(\alpha^{-1} x\right) F_{p}\left(\widetilde{R}_{\alpha}(x)\right)=0
$$

for all $x \in \mathbb{R}^{d}$, in the classical sense.

Proof. Let

$$
\begin{aligned}
g_{\alpha}(x):=-[ & q_{\alpha}(x) F_{p}^{\prime}\left(Q_{\mathbb{R}^{d}}(x)\right) \rho_{\alpha}(x)+\left[q_{\alpha}(x)-1\right] \mathcal{N}\left(\rho_{\alpha}\right)(x) \\
& \left.+\alpha^{-2} V_{d}\left(\alpha^{-1} x\right) Q_{\mathbb{R}^{d}}(x)+q_{\alpha}(x) F_{p}\left(Q_{\mathbb{R}^{d}}(x)\right)\right] .
\end{aligned}
$$

Then, by Lemma 3.6 , we have $g_{\alpha} \in L_{\text {rad }}^{2}\left(\mathbb{R}^{d}\right) \cap L^{\infty}\left(\mathbb{R}^{d}\right) \cap C\left(\mathbb{R}^{d}\right)$ and by construction, $\mathcal{A}_{\alpha} \rho_{\alpha}^{\sharp}=$ $g_{\alpha}^{\sharp}$. Equivalently, $\Delta_{\mathbb{R}^{d}} \rho_{\alpha}^{\sharp}=h_{\alpha}^{\sharp}$ with

$$
h_{\alpha}(x):=\rho_{\alpha}(x)-F_{p}^{\prime}\left(Q_{\mathbb{R}^{d}}(x)\right) \rho_{\alpha}(x)+\alpha^{-2} V_{d}\left(\alpha^{-1} x\right) \rho_{\alpha}(x)-g_{\alpha}(x) .
$$

Since $\rho_{\alpha}, h_{\alpha} \in C\left(\mathbb{R}^{d}\right)$ are radial, the claim follows from Lemma A.4.

Proof of Theorem 1.3. For $\alpha>0$ sufficiently large, let

$$
Q_{\mathbb{M}^{d}, \alpha}(r, y):=\alpha^{\frac{2}{p-1}}\left(\frac{r}{A(r)}\right)^{\frac{d-1}{2}}\left[Q_{\mathbb{R}^{d}}\left(\alpha r e_{1}\right)+\rho_{\alpha}\left(\alpha r e_{1}\right)\right] .
$$

By Lemma 3.9 and Hypothesis 1.1, $Q_{\mathbb{M}^{d}, \alpha} \in C^{2}\left(\mathbb{M}^{d}\right)$ and by construction, $u_{\alpha}^{*}(t, r, y)=$ $e^{i \alpha^{2} t} Q_{\mathbb{M}^{d}, \alpha}(r, y)$ solves Eq. (1.4) for all $t \in \mathbb{R}$. The remaining properties follow from Proposition 3.7 . 


\section{Spectral stability of the soliton}

In this section we investigate the linear stability of the soliton

$$
v_{\alpha}^{*}(t, x)=e^{i \alpha^{2} t} R_{\alpha}(x)=\alpha^{\frac{2}{p-1}} e^{i \alpha^{2} t}\left[Q_{\mathbb{R}^{d}}(\alpha x)+\rho_{\alpha}(\alpha x)\right]
$$

as a solution to the nonlinear Schrödinger equation (2.2). More precisely, we study spectral properties of the linearized operator $\mathcal{L}_{\alpha}$ associated to the soliton $v_{\alpha}^{*}$. We will see that the qualitative behavior is very similar to the Euclidean case.

4.1. The linearized operator. The notion of spectral stability derives from spectral properties of the operator that is obtained by linearizing Eq. (2.2) at the soliton $v_{\alpha}^{*}$. More precisely, we insert the ansatz

$$
v(t, x)=v_{\alpha}^{*}(t, x)+e^{i \alpha^{2} t} w(t, x)=e^{i \alpha^{2} t}\left[R_{\alpha}(x)+w(t, x)\right]
$$

into Eq. (2.2). This yields

$$
\begin{aligned}
i \partial_{t} w(t, \cdot) & +\Delta_{\mathbb{R}^{d}} w(t, \cdot)-\alpha^{2} w(t, \cdot)-V_{d} w(t, \cdot) \\
& +\varphi_{d, p} F_{p}\left(R_{\alpha}(\cdot)+w(t, \cdot)\right)-\varphi_{d, p} F_{p}\left(R_{\alpha}(\cdot)\right)=0,
\end{aligned}
$$

where we have used Eq. (2.3), i.e.,

$$
\Delta_{\mathbb{R}^{d}} R_{\alpha}-\alpha^{2} R_{\alpha}-V_{d} R_{\alpha}=-\varphi_{d, p} F_{p}\left(R_{\alpha}(\cdot)\right) .
$$

Now note that for all $a_{0}, a, b \in \mathbb{R}$,

$$
\begin{aligned}
\left|a_{0}+a+i b\right|^{p-1} & =\left(a_{0}^{2}+2 a_{0} a+a^{2}+b^{2}\right)^{\frac{p-1}{2}} \\
& =\left|a_{0}\right|^{p-1}+\frac{p-1}{2}\left(a_{0}^{2}\right)^{\frac{p-1}{2}-1}\left(2 a_{0} a+a^{2}+b^{2}\right)+N_{1}\left(a_{0}, a, b\right) \\
& =\left|a_{0}\right|^{p-1}+(p-1) a_{0}\left|a_{0}\right|^{p-3} a+N_{2}\left(a_{0}, a, b\right),
\end{aligned}
$$

where $N_{1}\left(a_{0}, a, b\right)$ and $N_{2}\left(a_{0}, a, b\right)$ are quadratic in $a$ and $b$. Hence,

$$
\begin{aligned}
F_{p}\left(a_{0}+a+i b\right) & =\left(a_{0}+a+i b\right)\left|a_{0}+a+i b\right|^{p-1} \\
& =a_{0}\left|a_{0}\right|^{p-1}+p\left|a_{0}\right|^{p-1} a+i\left|a_{0}\right|^{p-1} b+N\left(a_{0}, a, b\right),
\end{aligned}
$$

where $N\left(a_{0}, a, b\right)$ is quadratic in $a$ and $b$. This yields

$$
\begin{aligned}
F_{p}\left(R_{\alpha}(x)+w(t, x)\right)= & F_{p}\left(R_{\alpha}(x)+\operatorname{Re} w(t, x)+i \operatorname{Im} w(t, x)\right) \\
= & F_{p}\left(R_{\alpha}(x)\right)+p\left|R_{\alpha}(x)\right|^{p-1} \operatorname{Re} w(t, x)+i\left|R_{\alpha}(x)\right|^{p-1} \operatorname{Im} w(t, x) \\
& +N\left(R_{\alpha}(x), \operatorname{Re} w(t, x), \operatorname{Im} w(t, x)\right) .
\end{aligned}
$$

By dropping the nonlinear terms, we obtain from Eq. (4.1) the linearized problem

$$
\begin{aligned}
i \partial_{t} w(t, \cdot) & +\Delta_{\mathbb{R}^{d}} w(t, \cdot)-\alpha^{2} w(t, \cdot)-V_{d} w(t, \cdot) \\
& +p \varphi_{d, p}\left|R_{\alpha}(\cdot)\right|^{p-1} \operatorname{Re} w(t, \cdot)+i \varphi_{d, p}\left|R_{\alpha}(\cdot)\right|^{p-1} \operatorname{Im} w(t, \cdot)=0 .
\end{aligned}
$$

Finally, we rescale by setting $w(t, x)=\widetilde{w}\left(\alpha^{2} t, \alpha x\right)$. This yields

$$
\begin{aligned}
i \partial_{t} \widetilde{w}(t, x) & +\Delta_{\mathbb{R}^{d}, x} \widetilde{w}(t, x)-\widetilde{w}(t, x)-\alpha^{-2} V_{d}\left(\alpha^{-1} x\right) \widetilde{w}(t, x) \\
& +p \varphi_{d, p}\left(\alpha^{-1} x\right)\left|\widetilde{R}_{\alpha}(x)\right|^{p-1} \operatorname{Re} \widetilde{w}(t, x)+i \varphi_{d, p}\left(\alpha^{-1} x\right)\left|\widetilde{R}_{\alpha}(x)\right|^{p-1} \operatorname{Im} \widetilde{w}(t, \cdot)=0
\end{aligned}
$$


with $\widetilde{R}_{\alpha}(x)=\alpha^{-\frac{2}{p-1}} R_{\alpha}\left(\alpha^{-1} x\right)$. Eq. (4.2) is equivalent to the system

$$
\partial_{t}\left(\begin{array}{l}
\operatorname{Re} \widetilde{w}(t, \cdot) \\
\operatorname{Im} \widetilde{w}(t, \cdot)
\end{array}\right)=\mathcal{L}_{\alpha}\left(\begin{array}{l}
\operatorname{Re} \widetilde{w}(t, \cdot) \\
\operatorname{Im} \widetilde{w}(t, \cdot)
\end{array}\right)
$$

with the spatial differential operator

$$
\mathcal{L}_{\alpha}:=\left(\begin{array}{cc}
0 & \mathcal{L}_{\alpha,-} \\
-\mathcal{L}_{\alpha,+} & 0
\end{array}\right)
$$

where

$$
\begin{aligned}
& \mathcal{L}_{\alpha,-} f(x):=-\Delta_{\mathbb{R}^{d}} f(x)+f(x)-\varphi_{d, p}\left(\alpha^{-1} x\right)\left|\widetilde{R}_{\alpha}(x)\right|^{p-1} f(x)+\alpha^{-2} V_{d}\left(\alpha^{-1} x\right) f(x) \\
& \mathcal{L}_{\alpha,+} f(x):=-\Delta_{\mathbb{R}^{d}} f(x)+f(x)-p \varphi_{d, p}\left(\alpha^{-1} x\right)\left|\widetilde{R}_{\alpha}(x)\right|^{p-1} f(x)+\alpha^{-2} V_{d}\left(\alpha^{-1} x\right) f(x) .
\end{aligned}
$$

Consequently, (linear) stability properties of the soliton $v_{\alpha}^{*}$ are encoded in the spectrum of the operator $\mathcal{L}_{\alpha}$, which we consider on the space $L_{\text {rad }}^{2}\left(\mathbb{R}^{d}, \mathbb{C}^{2}\right)$. This is a natural choice since the operators $\mathcal{L}_{\alpha, \pm}$ are self-adjoint on $L_{\text {rad }}^{2}\left(\mathbb{R}^{d}\right)$. Formally at least, in the limit $\alpha \rightarrow \infty, \mathcal{L}_{\alpha}$ reduces to its well-known Euclidean counterpart $\mathcal{L}$, given by

$$
\mathcal{L}=\left(\begin{array}{cc}
0 & \mathcal{L}_{-} \\
-\mathcal{L}_{+} & 0
\end{array}\right)
$$

and

$$
\begin{aligned}
& \mathcal{L}_{-} f(x)=-\Delta_{\mathbb{R}^{d}} f(x)+f(x)-\left|Q_{\mathbb{R}^{d}}(x)\right|^{p-1} f(x) \\
& \mathcal{L}_{+} f(x)=-\Delta_{\mathbb{R}^{d}} f(x)+f(x)-p\left|Q_{\mathbb{R}^{d}}(x)\right|^{p-1} f(x) .
\end{aligned}
$$

This suggests a perturbative spectral analysis, based on the Euclidean situation.

4.2. Spectral properties in the Euclidean case. Our base case will be the Euclidean operator $\mathcal{L}$ which was extensively studied in the literature, see e.g. [70, 171, 35, 16]. Nevertheless, there are still a number of substantial questions that remain unanswered. We summarize some of the known results but restrict ourselves to the radial case. Since we will be dealing with spectra of nonself-adjoint operators, there are some ambiguities that need to be clarified first.

Definition 4.1. Let $T$ be a closed operator on a Banach space $X$. We define the essential spectrum $\sigma_{e}(T)$ of $T$ by

$$
\sigma_{e}(T):=\bigcap_{K \in \mathcal{K}(X)} \sigma(T+K)
$$

where $\mathcal{K}(X)$ denotes the set of all compact operators on $X$. Furthermore, $\sigma_{p}(T)$ is the set of all eigenvalues of $T$.

Remark 4.2. There are other meaningful definitions of essential spectra for nonself-adjoint operators in the literature, see e.g. [38, 32, 42] for a discussion on this. The choice we made is the largest possible that is invariant under relatively compact perturbations. However, for the particular class of operators we will be concerned with, all the usual definitions turn out to be equivalent, see [42].

Theorem $4.3\left(\left[70\right.\right.$, 71, 35, 16, 59]). The operator $\mathcal{L}: H_{\mathrm{rad}}^{2}\left(\mathbb{R}^{d}, \mathbb{C}^{2}\right) \subset L_{\mathrm{rad}}^{2}\left(\mathbb{R}^{d}, \mathbb{C}^{2}\right) \rightarrow$ $L_{\mathrm{rad}}^{2}\left(\mathbb{R}^{d}, \mathbb{C}^{2}\right)$ is closed and has the following properties: 
- The spectrum $\sigma(\mathcal{L})$ is a subset of $\mathbb{R} \cup i \mathbb{R}$.

- If $\lambda \in \sigma(\mathcal{L})$ then $-\lambda \in \sigma(\mathcal{L})$.

- The essential spectrum of $\mathcal{L}$ is given by

$$
\sigma_{e}(\mathcal{L})=\{z \in \mathbb{C}: \operatorname{Re} z=0,|\operatorname{Im} z| \geq 1\} .
$$

- The set $\sigma(\mathcal{L}) \backslash \sigma_{e}(\mathcal{L})$ is free of accumulation points and consists of eigenvalues with finite algebraic multiplicities.

- We have $0 \in \sigma_{p}(\mathcal{L})$ and

$$
\operatorname{ker} \mathcal{L}=\left\langle\left(\begin{array}{c}
0 \\
Q_{\mathbb{R}^{d}}
\end{array}\right)\right\rangle .
$$

- For the kernels of powers of $\mathcal{L}$ we have

$$
\operatorname{dim} \operatorname{ker}\left(\mathcal{L}^{2}\right)=\operatorname{dim} \operatorname{ker}\left(\mathcal{L}^{3}\right)=\left\{\begin{array}{ll}
2 & \text { if } p \neq 1+\frac{4}{d} \\
4 & \text { if } p=1+\frac{4}{d}
\end{array} .\right.
$$

In particular, the algebraic multiplicity of the eigenvalue $0 \in \sigma_{p}(\mathcal{L})$ equals 4 in the $L^{2}$-critical case $p=1+\frac{4}{d}$ and 2 otherwise.

- In the $L^{2}$-subcritical case $p<1+\frac{4}{d}, \mathcal{L}$ has no positive eigenvalues. In the $L^{2}$ supercritical case $p>1+\frac{4}{d}, \mathcal{L}$ has precisely one positive eigenvalue $\lambda$ and the eigenvalues $\pm \lambda$ are simple.

Remark 4.4. The picture one has in mind is as follows. Starting from the supercritical case $p>1+\frac{4}{d}$, the two nonzero real eigenvalues move towards the origin as $p$ decreases. Precisely when $p=1+\frac{4}{d}$, the two eigenvalues merge and the algebraic multiplicity of $0 \in \sigma_{p}(\mathcal{L})$ increases by two. If one decreases $p$ further into the subcritical regime $p<1+\frac{4}{d}$, a pair of purely imaginary eigenvalues emerges from 0 . In particular, the ground state is linearly stable in the subcritical case and unstable in the supercritical case. These linear stability properties are reflected in the nonlinear theory. Indeed, in the subcritical case the ground state is orbitally stable and in the supercritical case it is unstable. The critical case $p=1+\frac{4}{d}$ is more delicate as there is spectral stability (that is to say, no spectrum away from the imaginary axis) but quite strong instability in the nonlinear theory.

Remark 4.5. Important issues that remain unsolved concern the existence of eigenvalues and/or resonances embedded in the essential spectrum and the "gap property". The latter refers to the absence of eigenvalues on the imaginary axis between 0 and $i$ in the supercritical case. These spectral properties are important for the (nonlinear) asymptotic stability theory of the ground state. Some of them have been verified numerically or even proved rigorously in special cases, see e.g. [31, 46, 16, 24, but there is no systematic theoretical understanding so far.

4.3. Spectral properties in the curved geometry. To begin, we show that the structural properties of the spectrum in the curved case are the same as in the Euclidean case. An important prerequisite is the nonnegativity of $\mathcal{L}_{\alpha,-}$, which we establish first.

Proposition 4.6. There exists an $\alpha_{0}>0$ such that, for all $\alpha \geq \alpha_{0}, \mathcal{L}_{\alpha,-}: H_{\text {rad }}^{2}\left(\mathbb{R}^{d}\right) \subset$ $L_{\mathrm{rad}}^{2}\left(\mathbb{R}^{d}\right) \rightarrow L_{\mathrm{rad}}^{2}\left(\mathbb{R}^{d}\right)$ is self-adjoint with the following properties:

- The essential spectrum of $\mathcal{L}_{\alpha,-}$ is given by $\sigma_{e}\left(\mathcal{L}_{\alpha,-}\right)=\left[1+V_{0, d} \alpha^{-2}, \infty\right)$.

- We have $0 \in \sigma_{p}\left(\mathcal{L}_{\alpha,-}\right)$ and $\operatorname{ker} \mathcal{L}_{\alpha,-}=\left\langle Q_{\mathbb{R}^{d}}+\rho_{\alpha}\right\rangle$. 
- The operator $\mathcal{L}_{\alpha,-}$ satisfies

$$
\left(\mathcal{L}_{\alpha,-} f \mid f\right)_{L^{2}\left(\mathbb{R}^{d}\right)} \gtrsim\|f\|_{L^{2}\left(\mathbb{R}^{d}\right)}^{2}
$$

for all $f \in\left\langle Q_{\mathbb{R}^{d}}+\rho_{\alpha}\right\rangle^{\perp} \cap H_{\text {rad }}^{2}\left(\mathbb{R}^{d}\right)$ and all $\alpha \geq \alpha_{0}$.

Proof. We define $\mathcal{L}_{\alpha, 0}: H_{\mathrm{rad}}^{2}\left(\mathbb{R}^{d}\right) \subset L_{\mathrm{rad}}^{2}\left(\mathbb{R}^{d}\right) \rightarrow L_{\mathrm{rad}}^{2}\left(\mathbb{R}^{d}\right)$ by $\mathcal{L}_{\alpha, 0} f:=-\Delta_{\mathbb{R}^{d}} f+\left(1+V_{0, d} \alpha^{-2}\right) f$ and set

$$
W_{\alpha}(x):=-\varphi_{d, p}\left(\alpha^{-1} x\right)\left|Q_{\mathbb{R}^{d}}(x)+\rho_{\alpha}(x)\right|^{p-1}+\alpha^{-2}\left[V_{d}\left(\alpha^{-1} x\right)-V_{0, d}\right] .
$$

Then we have $\mathcal{L}_{\alpha,-} f=\mathcal{L}_{\alpha, 0} f+W_{\alpha} f$. By Fourier analysis it follows that

$$
\sigma\left(\mathcal{L}_{\alpha, 0}\right)=\sigma_{e}\left(\mathcal{L}_{\alpha, 0}\right)=\left[1+V_{0, d} \alpha^{-2}, \infty\right) .
$$

Furthermore, by Hypothesis 1.1, Lemma 3.5, Proposition 3.7, and Lemma 3.8, we have $W_{\alpha} \in L^{\infty}\left(\mathbb{R}^{d}\right) \cap C\left(\mathbb{R}^{d}\right)$ and

$$
\lim _{|x| \rightarrow \infty} W_{\alpha}(x)=0 .
$$

Consequently, $f \mapsto W_{\alpha} f: L_{\text {rad }}^{2}\left(\mathbb{R}^{d}\right) \rightarrow L_{\text {rad }}^{2}\left(\mathbb{R}^{d}\right)$ is bounded and the Kato-Rellich theorem (see e.g. 68], p. 159, Theorem 6.4) shows that $\mathcal{L}_{\alpha,-}$ is self-adjoint. In particular, $\sigma\left(\mathcal{L}_{\alpha,-}\right) \subset \mathbb{R}$. Furthermore, by [68], p. 258, Theorem 10.2, the operator $f \mapsto W_{\alpha} f: L_{\mathrm{rad}}^{2}\left(\mathbb{R}^{d}\right) \rightarrow L_{\mathrm{rad}}^{2}\left(\mathbb{R}^{d}\right)$ is relatively compact with respect to $\mathcal{L}_{\alpha, 0}$ and Weyl's theorem (see e.g. [68], p. 171, Theorem 6.19) implies that $\sigma_{e}\left(\mathcal{L}_{\alpha,-}\right)=\left[1+V_{0, d} \alpha^{-2}, \infty\right)$. As a consequence, $\sigma\left(\mathcal{L}_{\alpha,-}\right) \backslash \sigma_{e}\left(\mathcal{L}_{\alpha,-}\right)$ consists of isolated eigenvalues only. The same is true for the limiting operator $\mathcal{L}_{-}$, i.e., $\sigma\left(\mathcal{L}_{-}\right) \backslash \sigma_{e}\left(\mathcal{L}_{-}\right)$ consists of isolated eigenvalues only, where $\sigma_{e}\left(\mathcal{L}_{-}\right)=[1, \infty)$.

Next, we show that there exists a constant $\mu>0$ such that

$$
(-\infty,-\mu) \subset \rho\left(\mathcal{L}_{\alpha,-}\right)
$$

for all $\alpha \geq \alpha_{0}$. To this end we use the resolvent bound $\left\|\left(\lambda-\mathcal{L}_{0, \alpha}\right)^{-1}\right\|_{L^{2}\left(\mathbb{R}^{d}\right)} \leq|\lambda|^{-1}$, valid for all $\lambda<0$, which is a consequence of the self-adjointness of $\mathcal{L}_{\alpha, 0}$ and $\sigma\left(\mathcal{L}_{\alpha, 0}\right) \subset\left[1+V_{0, d} \alpha^{-2}, \infty\right)$. Furthermore, we note that the operator $\mathcal{B}_{\alpha} f:=W_{\alpha} f$ satisfies

$$
\left\|\mathcal{B}_{\alpha} f\right\|_{L^{2}\left(\mathbb{R}^{d}\right)} \leq\left\|W_{\alpha}\right\|_{L^{\infty}\left(\mathbb{R}^{d}\right)}\|f\|_{L^{2}\left(\mathbb{R}^{d}\right)} \lesssim\|f\|_{L^{2}\left(\mathbb{R}^{d}\right)}
$$

for all $\alpha \geq \alpha_{0}$ and $f \in L_{\mathrm{rad}}^{2}\left(\mathbb{R}^{d}\right)$. Consequently, if $\mu>0$ is sufficiently large, the operator $1-\mathcal{B}_{\alpha}\left(\lambda-\mathcal{L}_{\alpha, 0}\right)^{-1}$ is invertible for all $\lambda<-\mu$ by a Neumann series argument and the identity,

$$
\lambda-\mathcal{L}_{\alpha,-}=\left[1-\mathcal{B}_{\alpha}\left(\lambda-\mathcal{L}_{\alpha, 0}\right)^{-1}\right]\left(\lambda-\mathcal{L}_{\alpha, 0}\right),
$$

proves (4.4).

Now we turn to the computation of ker $\mathcal{L}_{\alpha,-}$. Obviously, $0 \in \sigma_{p}\left(\mathcal{L}_{\alpha,-}\right)$ since $\widetilde{R}_{\alpha}=Q_{\mathbb{R}^{d}}+$ $\rho_{\alpha} \in H_{\mathrm{rad}}^{2}\left(\mathbb{R}^{d}\right) \cap C^{2}\left(\mathbb{R}^{d}\right)$ by Lemma 3.9 and

$$
\begin{aligned}
\mathcal{L}_{\alpha,-} \widetilde{R}_{\alpha}(x) & =-\Delta_{\mathbb{R}^{d}} \widetilde{R}_{\alpha}(x)+\widetilde{R}_{\alpha}(x)-\varphi_{d, p}\left(\alpha^{-1} x\right)\left|\widetilde{R}_{\alpha}(x)\right|^{p-1} \widetilde{R}_{\alpha}(x)+\alpha^{-2} V_{d}\left(\alpha^{-1} x\right) \widetilde{R}_{\alpha}(x) \\
& =0 .
\end{aligned}
$$

In particular, $\left\langle\widetilde{R}_{\alpha}\right\rangle \subset \operatorname{ker}\left(\mathcal{L}_{\alpha,-}\right)$. To prove the reverse inclusion, suppose $f \in H_{\text {rad }}^{2}\left(\mathbb{R}^{d}\right) \backslash\{0\}$ satisfies $\mathcal{L}_{\alpha,-} f=0$. By the one-dimensional Sobolev embedding we have $f \in C\left(\mathbb{R}^{d} \backslash\{0\}\right)$ 
and thus, $W_{\alpha} f \in L_{\text {rad }}^{2}\left(\mathbb{R}^{d}\right) \cap C\left(\mathbb{R}^{d} \backslash\{0\}\right)$. Consequently, Lemma A.3 implies that $\widehat{f}(r):=$ $r^{\frac{d-1}{2}} f\left(r e_{1}\right)$ belongs to $C^{2}(0, \infty)$ and satisfies

$$
\widehat{f}^{\prime \prime}(r)-\frac{(d-1)(d-3)}{4 r^{2}} \widehat{f}(r)-\left[1+V_{0, d} \alpha^{-2}\right] \widehat{f}(r)=W_{\alpha}\left(r e_{1}\right) \widehat{f}(r)
$$

for all $r>0$. According to Lemma B.1, there exist constants $a, b \in \mathbb{C}$ such that $\widehat{f}(r)=$ $a \phi_{0}(r)+b \psi_{0}(r)$, where $\left|\phi_{0}(r)\right| \simeq r^{\frac{d-1}{2}},\left|\phi_{0}^{\prime}(r)\right| \simeq r^{\frac{d-3}{2}}$, and

$$
\left|\psi_{0}(r)\right| \simeq\left\{\begin{array}{ll}
r^{\frac{1}{2}|\log r|} & d=2 \\
r^{-\frac{d-3}{2}} & d \neq 2
\end{array}, \quad\left|\psi_{0}^{\prime}(r)\right| \simeq \begin{cases}r^{-\frac{1}{2}}|\log r| & d=2 \\
r^{-\frac{d-1}{2}} & d \neq 2\end{cases}\right.
$$

for $r \in\left(0, \frac{1}{2}\right]$. Since $f \in H_{\text {rad }}^{1}\left(\mathbb{R}^{d}\right)$, we must have $b=0$ and this shows that the kernel of $\mathcal{L}_{\alpha,-}$ is one-dimensional. Consequently, $\operatorname{ker} \mathcal{L}_{\alpha,-}=\left\langle\widetilde{R}_{\alpha}\right\rangle$, as claimed.

Now we define an operator $\mathcal{C}_{\alpha}: L_{\text {rad }}^{2}\left(\mathbb{R}^{d}\right) \rightarrow L_{\text {rad }}^{2}\left(\mathbb{R}^{d}\right)$ such that $\mathcal{L}_{\alpha,-}=\mathcal{L}_{-}+\mathcal{C}_{\alpha}$, i.e.,

$$
\begin{aligned}
\mathcal{C}_{\alpha} f(x) & =\mathcal{L}_{\alpha,-} f(x)-\mathcal{L}_{-} f(x) \\
& =-\varphi_{d, p}\left(\alpha^{-1} x\right)\left|Q_{\mathbb{R}^{d}}(x)+\rho_{\alpha}(x)\right|^{p-1} f(x)+\left|Q_{\mathbb{R}^{d}}(x)\right|^{p-1} f(x)+\alpha^{-2} V_{d}\left(\alpha^{-1} x\right) f(x) \\
& =: U_{\alpha}(x) f(x) .
\end{aligned}
$$

We have

$$
\begin{aligned}
\left|U_{\alpha}(x)\right| \lesssim & || Q_{\mathbb{R}^{d}}(x)+\left.\rho_{\alpha}(x)\right|^{p-1}-\left|Q_{\mathbb{R}^{d}}(x)\right|^{p-1}|+| q_{\alpha}(x)|| Q_{\mathbb{R}^{d}}(x)+\left.\rho_{\alpha}(x)\right|^{p-1} \\
& +\alpha^{-2}\left|V_{d}\left(\alpha^{-1} x\right)\right| \\
\lesssim & \left|\rho_{\alpha}(x)\right|^{p-1}+\left|\rho_{\alpha}(x)\right|+\left|q_{\alpha}(x) F_{p}^{\prime}\left(Q_{\mathbb{R}^{d}}(x)\right)\right|+\left|q_{\alpha}(x)\right|\left|\rho_{\alpha}(x)\right|^{p-1}+\alpha^{-2} \\
\lesssim & \alpha^{-(p-1)}+\alpha^{-1}+\alpha^{-2}
\end{aligned}
$$

for all $x \in \mathbb{R}^{d}$ by Lemma 3.5 and Proposition 3.7. Here we have used the elementary estimates (3.5) and (3.6) from the proof of Lemma 3.6. Consequently, $\left\|U_{\alpha}\right\|_{L^{\infty}\left(\mathbb{R}^{d}\right)} \rightarrow 0$ as $\alpha \rightarrow \infty$ and this shows that the operator $\mathcal{C}_{\alpha}$ converges to 0 in norm as $\alpha \rightarrow \infty$. Recall that $\mathcal{L}_{-}$is nonnegative. This is a consequence of $Q_{\mathbb{R}^{d}}>0, \mathcal{L}_{-} Q_{\mathbb{R}^{d}}=0$, and the Sturm oscillation theorem. Let $d_{0}:=\operatorname{dist}\left(0, \sigma\left(\mathcal{L}_{-}\right) \backslash\{0\}\right)$. Since 0 is an isolated eigenvalue of $\mathcal{L}_{-}$, we have $d_{0}>0$. Let $\gamma:[0,2 \pi] \rightarrow \mathbb{C}$ be a simple, closed, smooth curve that encircles the interval $\left[-1-\mu, \frac{1}{4} d_{0}\right]$ and such that $\gamma(t) \cap\left[\frac{3}{4} d_{0}, \infty\right)=\emptyset$ for all $t \in[0,2 \pi]$. By construction, $\gamma(t) \in \rho\left(\mathcal{L}_{-}\right)$for all $t \in[0,2 \pi]$ and thus, the spectral projection

$$
\mathcal{P}:=\frac{1}{2 \pi i} \int_{\gamma}\left(z-\mathcal{L}_{-}\right)^{-1} d z
$$

is well defined. By the self-adjointness of $\mathcal{L}_{-}$, we have $\operatorname{rg} \mathcal{P}=\operatorname{ker} \mathcal{L}_{-}=\left\langle Q_{\mathbb{R}^{d}}\right\rangle$ since 0 is the only spectral point of $\mathcal{L}_{-}$inside of $\gamma$. Recall that $\mathcal{C}_{\alpha} \rightarrow 0$ in norm as $\alpha \rightarrow \infty$ and thus, $\gamma(t) \in \rho\left(\mathcal{L}_{\alpha,-}\right)$ for all $t \in[0,2 \pi]$ and $\alpha \geq \alpha_{0}$, provided $\alpha_{0}>0$ is sufficiently large. This follows immediately from the identity

$$
z-\mathcal{L}_{\alpha,-}=\left[1-\mathcal{C}_{\alpha}\left(z-\mathcal{L}_{-}\right)^{-1}\right]\left(z-\mathcal{L}_{-}\right),
$$

valid for all $z \in \rho\left(\mathcal{L}_{-}\right)$. Thus, the spectral projection

$$
\mathcal{P}_{\alpha}:=\frac{1}{2 \pi i} \int_{\gamma}\left(z-\mathcal{L}_{\alpha,-}\right)^{-1} d z
$$


is well defined, and we have $\mathcal{P}_{\alpha} \rightarrow \mathcal{P}$ in norm as $\alpha \rightarrow \infty$. Consequently, by [43], p. 34, Lemma 4.10, it follows that $\operatorname{dim} \operatorname{rg} \mathcal{P}_{\alpha}=\operatorname{dim} \operatorname{rg} \mathcal{P}=1$ for all $\alpha \geq \alpha_{0}$. Since $0 \in \sigma_{p}\left(\mathcal{L}_{\alpha,-}\right)$, we conclude that 0 is the only spectral point of $\mathcal{L}_{\alpha,-}$ in the interval $\left[-1-\mu, \frac{1}{4} d_{0}\right]$. Finally, with (4.4), we infer that $\left(-\infty, \frac{1}{4} d_{0}\right] \cap \sigma\left(\mathcal{L}_{\alpha,-}\right)=\{0\}$ for all $\alpha \geq \alpha_{0}$. In particular, $\mathcal{L}_{\alpha,-}$ is nonnegative, and this finishes the proof.

We also note the following simple observation concerning the operator $\mathcal{L}_{\alpha,+}$.

Lemma 4.7. The operator $\mathcal{L}_{\alpha,+}: H_{\mathrm{rad}}^{2}\left(\mathbb{R}^{d}\right) \subset L_{\mathrm{rad}}^{2}\left(\mathbb{R}^{d}\right) \rightarrow L_{\mathrm{rad}}^{2}\left(\mathbb{R}^{d}\right)$ is self-adjoint and invertible.

Proof. We write $\mathcal{L}_{\alpha,+}=\mathcal{L}_{+}+\mathcal{B}_{\alpha}$ with $\mathcal{B}_{\alpha} f(x)=W_{\alpha}(x) f(x)$ and

$$
W_{\alpha}(x)=-p\left[\varphi_{d, p}\left(\alpha^{-1} x\right)\left|Q_{\mathbb{R}^{d}}(x)+\rho_{\alpha}(x)\right|^{p-1}-\left|Q_{\mathbb{R}^{d}}(x)\right|^{p-1}\right]+\alpha^{-2} V_{d}\left(\alpha^{-1} x\right) .
$$

We have $\left\|W_{\alpha}\right\|_{L^{\infty}\left(\mathbb{R}^{d}\right)} \rightarrow 0$ as $\alpha \rightarrow \infty$ (cf. the proof of Proposition 4.6) and thus, $\mathcal{B}_{\alpha}$ is a bounded symmetric operator on $L_{\text {rad }}^{2}\left(\mathbb{R}^{d}\right)$ that converges to 0 in norm as $\alpha \rightarrow \infty$. Consequently, the Kato-Rellich theorem implies that $\mathcal{L}_{\alpha,+}$ is self-adjoint. Since $0 \in \rho\left(\mathcal{L}_{+}\right)$, it follows from the identity

$$
\mathcal{L}_{\alpha,+}=\left[1+\mathcal{B}_{\alpha} \mathcal{L}_{+}^{-1}\right] \mathcal{L}_{+}
$$

and a Neumann series argument, that $\mathcal{L}_{\alpha,+}$ is invertible for all $\alpha \geq \alpha_{0}$, provided $\alpha_{0}>0$ is sufficiently large.

Based on the results on $\mathcal{L}_{\alpha, \pm}$, we can now establish some basic structural properties concerning the spectrum of $\mathcal{L}_{\alpha}$.

Lemma 4.8. There exists an $\alpha_{0}>0$ such that for all $\alpha \geq \alpha_{0}$ the operator $\mathcal{L}_{\alpha}: H_{\text {rad }}^{2}\left(\mathbb{R}^{d}, \mathbb{C}^{2}\right) \subset$ $L_{\mathrm{rad}}^{2}\left(\mathbb{R}^{d}, \mathbb{C}^{2}\right) \rightarrow L_{\mathrm{rad}}^{2}\left(\mathbb{R}^{d}, \mathbb{C}^{2}\right)$ is closed and the following holds:

- The spectrum of $\mathcal{L}_{\alpha}$ is a subset of $\mathbb{R} \cup i \mathbb{R}$.

- If $\lambda \in \sigma\left(\mathcal{L}_{\alpha}\right)$ then $-\lambda \in \sigma\left(\mathcal{L}_{\alpha}\right)$.

- The essential spectrum of $\mathcal{L}_{\alpha}$ is given by

$$
\sigma_{e}\left(\mathcal{L}_{\alpha}\right)=\left\{z \in \mathbb{C}: \operatorname{Re} z=0,|\operatorname{Im} z| \geq 1+V_{0, d} \alpha^{-2}\right\} .
$$

- There exists a $\mu>0$ (independent of $\alpha$ ) such that $(-\infty,-\mu) \cup(\mu, \infty) \subset \rho\left(\mathcal{L}_{\alpha}\right)$.

- The set $\sigma\left(\mathcal{L}_{\alpha}\right) \backslash \sigma_{e}\left(\mathcal{L}_{\alpha}\right)$ is free of accumulation points and consists of eigenvalues with finite algebraic multiplicities.

- We have $0 \in \sigma_{p}\left(\mathcal{L}_{\alpha}\right)$ and

$$
\operatorname{ker} \mathcal{L}_{\alpha}=\left\langle\left(\begin{array}{c}
0 \\
Q_{\mathbb{R}^{d}}+\rho_{\alpha}
\end{array}\right)\right\rangle .
$$

Proof. First of all, $\mathcal{L}_{\alpha, \pm}$ are self-adjoint and hence closed. This implies the closedness of $\mathcal{L}_{\alpha}$. Now consider the unitary operator $\mathcal{U}: L^{2}\left(\mathbb{R}^{d}, \mathbb{C}^{2}\right) \rightarrow L^{2}\left(\mathbb{R}^{d}, \mathbb{C}^{2}\right)$ given by

$$
\mathcal{U}:=\frac{1}{\sqrt{2}}\left(\begin{array}{cc}
1 & i \\
1 & -i
\end{array}\right)
$$

and set $\mathcal{H}_{\alpha}:=i \mathcal{U} \mathcal{L}_{\alpha} \mathcal{U}^{*}$. Explicitly, we have

$$
\mathcal{H}_{\alpha}=i \mathcal{U} \mathcal{L}_{\alpha} \mathcal{U}^{*}=\frac{1}{2}\left(\begin{array}{cc}
\mathcal{L}_{\alpha,-}+\mathcal{L}_{\alpha,+} & -\mathcal{L}_{\alpha,-}+\mathcal{L}_{\alpha,+} \\
\mathcal{L}_{\alpha,-}-\mathcal{L}_{\alpha,+} & -\mathcal{L}_{\alpha,-}-\mathcal{L}_{\alpha,+}
\end{array}\right)=\mathcal{H}_{0, \alpha}+\mathcal{H}_{\alpha}^{\prime}
$$


where

$$
\begin{aligned}
\mathcal{H}_{0, \alpha} & :=\left(\begin{array}{cc}
-\Delta_{\mathbb{R}^{d}}+1+V_{0, d} \alpha^{-2} & 0 \\
0 & \Delta_{\mathbb{R}^{d}}-1-V_{0, d} \alpha^{-2}
\end{array}\right), \\
\mathcal{H}_{\alpha}^{\prime}\left(\begin{array}{c}
f_{1} \\
f_{2}
\end{array}\right) & :=\left(\begin{array}{cc}
U_{\alpha} & W_{\alpha} \\
-W_{\alpha} & -U_{\alpha}
\end{array}\right)\left(\begin{array}{l}
f_{1} \\
f_{2}
\end{array}\right),
\end{aligned}
$$

and

$$
\begin{aligned}
U_{\alpha}(x) & :=-\frac{p+1}{2} \varphi_{d, p}\left(\alpha^{-1} x\right)\left|Q_{\mathbb{R}^{d}}(x)+\rho_{\alpha}(x)\right|^{p-1}+\alpha^{-2}\left[V_{d}\left(\alpha^{-1} x\right)-V_{0, d}\right], \\
W_{\alpha}(x) & :=-\frac{p-1}{2} \varphi_{d, p}\left(\alpha^{-1} x\right)\left|Q_{\mathbb{R}^{d}}(x)+\rho_{\alpha}(x)\right|^{p-1} .
\end{aligned}
$$

Evidently, $\mathcal{H}_{0, \alpha}$ is self-adjoint, and

$$
\sigma\left(\mathcal{H}_{0, \alpha}\right)=\sigma_{e}\left(\mathcal{H}_{0, \alpha}\right)=\left(-\infty,-1-V_{0, d} \alpha^{-2}\right] \cup\left[1+V_{0, d} \alpha^{-2}, \infty\right) .
$$

Furthermore, $\mathcal{H}_{\alpha}^{\prime}$ is bounded, and $U_{\alpha}, W_{\alpha} \in L^{\infty}\left(\mathbb{R}^{d}\right) \cap C\left(\mathbb{R}^{d}\right)$ with

$$
\lim _{|x| \rightarrow \infty} U_{\alpha}(x)=\lim _{|x| \rightarrow \infty} W_{\alpha}(x)=0
$$

by Hypothesis 1.1, Proposition 3.7, and Lemma 3.8. By [68], p. 201, Lemma 7.21, we see that $\mathcal{H}_{\alpha}^{\prime}\left(z-\mathcal{H}_{0, \alpha}\right)^{-1}$ is compact for any $z \in \rho\left(\mathcal{H}_{0, \alpha}\right)$. In other words, $\mathcal{H}_{\alpha}^{\prime}$ is relatively compact with respect to $\mathcal{H}_{0, \alpha}$. Consequently, by [58], p. 173, Theorem 7.28,

$$
\sigma_{e}\left(\mathcal{H}_{\alpha}\right)=\sigma_{e}\left(\mathcal{H}_{0, \alpha}+\mathcal{H}_{\alpha}^{\prime}\right)=\sigma_{e}\left(\mathcal{H}_{0, \alpha}\right)=\left(-\infty,-1-V_{0, d} \alpha^{-2}\right] \cup\left[1+V_{0, d} \alpha^{-2}, \infty\right),
$$

and, since $\mathcal{H}_{\alpha}$ is unitarily equivalent to $i \mathcal{L}_{\alpha}$, the statement on $\sigma_{e}\left(\mathcal{L}_{\alpha}\right)$ follows.

From the identity

$$
z-\mathcal{H}_{\alpha}=\left[1-\mathcal{H}_{\alpha}^{\prime}\left(z-\mathcal{H}_{0, \alpha}\right)^{-1}\right]\left(z-\mathcal{H}_{0, \alpha}\right), \quad z \in \rho\left(\mathcal{H}_{0, \alpha}\right),
$$

we infer that $z-\mathcal{H}_{\alpha}$ is invertible for $z \in \rho\left(\mathcal{H}_{0, \alpha}\right)$ if and only if $1-\mathcal{H}_{\alpha}^{\prime}\left(z-\mathcal{H}_{0, \alpha}\right)^{-1}$ is invertible. By the self-adjointness of $\mathcal{H}_{0, \alpha}$ we have the bound $\left\|\left(z-\mathcal{H}_{0, \alpha}\right)^{-1}\right\|_{L^{2}\left(\mathbb{R}^{d}, \mathbb{C}^{2}\right)} \leq|\operatorname{Im} z|^{-1}$ and thus, $z-\mathcal{H}_{\alpha}$ is certainly invertible for all $z$ sufficiently far away from the real axis. Furthermore, $\left\|\mathcal{H}_{\alpha}^{\prime}\right\|_{L^{2}\left(\mathbb{R}^{d}, \mathbb{C}^{2}\right)} \lesssim 1$ for all $\alpha \geq \alpha_{0}$, and thus, there exists a $\mu>0$ such that

$$
\{z \in \mathbb{C}: \operatorname{Re} z=0,|\operatorname{Im} z|>\mu\} \subset \rho\left(\mathcal{H}_{\alpha}\right)
$$

for all $\alpha \geq \alpha_{0}$. Consequently, the analytic Fredholm theorem (see e.g. [62], p. 194, Theorem 3.14.3) applied to $\mathcal{H}_{\alpha}^{\prime}\left(z-\mathcal{H}_{0, \alpha}\right)^{-1}$ shows that $\sigma\left(\mathcal{H}_{\alpha}\right) \backslash \sigma_{e}\left(\mathcal{H}_{\alpha}\right)$ consists of isolated eigenvalues of finite algebraic multiplicities which do not accumulate at any point outside of $\sigma_{e}\left(\mathcal{H}_{\alpha}\right)$.

Next, we turn to the proof that $\sigma\left(\mathcal{L}_{\alpha}\right) \subset \mathbb{R} \cup i \mathbb{R}$. Since $\sigma_{e}\left(\mathcal{L}_{\alpha}\right) \subset i \mathbb{R}$ and $\sigma\left(\mathcal{L}_{\alpha}\right) \backslash \sigma_{e}\left(\mathcal{L}_{\alpha}\right)$ consists of eigenvalues only, it suffices to prove that $\sigma_{p}\left(\mathcal{L}_{\alpha}\right) \subset \mathbb{R} \cup i \mathbb{R}$. Furthermore, we may restrict ourselves to nonzero eigenvalues. Let $\lambda \in \sigma_{p}\left(\mathcal{L}_{\alpha}\right) \backslash\{0\}$ with eigenfunction $f=\left(f_{1}, f_{2}\right) \in H^{2}\left(\mathbb{R}^{d}, \mathbb{C}^{2}\right)$. The eigenvalue equation $\left(\lambda-\mathcal{L}_{\alpha}\right) f=0$ is equivalent to

$$
\left\{\begin{array}{l}
\mathcal{L}_{\alpha,-} f_{2}=\lambda f_{1} \\
\mathcal{L}_{\alpha,+} f_{1}=-\lambda f_{2}
\end{array}\right.
$$

Let $\mathcal{P}_{\alpha}: L_{\text {rad }}^{2}\left(\mathbb{R}^{d}\right) \rightarrow L_{\text {rad }}^{2}\left(\mathbb{R}^{d}\right)$ be the orthogonal projection onto $\left\langle Q_{\mathbb{R}^{d}}+\rho_{\alpha}\right\rangle$ and set $\mathcal{P}_{\alpha}^{\perp}:=$ $1-\mathcal{P}_{\alpha}$. Note that we must have $\mathcal{P}_{\alpha}^{\perp} f_{2} \neq 0$ because otherwise,

$$
f_{1}=\frac{1}{\lambda} \mathcal{L}_{\alpha,-}\left(\mathcal{P}_{\alpha} f_{2}+\underset{22}{\mathcal{\perp}} f_{2}\right)=\frac{1}{\lambda} \mathcal{L}_{\alpha,-} \mathcal{P}_{\alpha} f_{2}=0,
$$


by Proposition 4.6, and from the second equation in (4.5) we infer that $f_{2}=0$. This is a contradiction to $f=\left(f_{1}, f_{2}\right)$ being an eigenfunction. Note further that $\mathcal{P}_{\alpha}$ is the spectral projection associated to the eigenvalue $0 \in \sigma_{p}\left(\mathcal{L}_{\alpha,-}\right)$ and thus, $\mathcal{P}_{\alpha}$ commutes with $\mathcal{L}_{\alpha,-}$. From the first equation in (4.5) and Proposition 4.6 we obtain

$$
0 \neq\left(\mathcal{L}_{\alpha,-} \mathcal{P}_{\alpha}^{\perp} f_{2} \mid \mathcal{P}_{\alpha}^{\perp} f_{2}\right)_{L^{2}\left(\mathbb{R}^{d}\right)}=\left(\mathcal{L}_{\alpha,-} f_{2} \mid f_{2}\right)_{L^{2}\left(\mathbb{R}^{d}\right)}=\lambda\left(f_{1} \mid f_{2}\right)_{L^{2}\left(\mathbb{R}^{d}\right)},
$$

and the second equation in (4.5) yields

$$
\left(\mathcal{L}_{\alpha,+} f_{1} \mid f_{1}\right)_{L^{2}\left(\mathbb{R}^{d}\right)}=-\lambda\left(f_{2} \mid f_{1}\right)_{L^{2}\left(\mathbb{R}^{d}\right)}=-\lambda \overline{\left(f_{1} \mid f_{2}\right)_{L^{2}\left(\mathbb{R}^{d}\right)}} .
$$

Consequently, since $\left(f_{1} \mid f_{2}\right)_{L^{2}\left(\mathbb{R}^{d}\right)} \neq 0$,

$$
\lambda^{2}=-\frac{\left(\mathcal{L}_{\alpha,-} f_{2} \mid f_{2}\right)_{L^{2}\left(\mathbb{R}^{d}\right)}\left(\mathcal{L}_{\alpha,+} f_{1} \mid f_{1}\right)_{L^{2}\left(\mathbb{R}^{d}\right)}}{\left|\left(f_{1} \mid f_{2}\right)_{L^{2}\left(\mathbb{R}^{d}\right)}\right|^{2}} \in \mathbb{R},
$$

which implies that $\lambda \in \mathbb{R} \cup i \mathbb{R}$. From Eq. (4.5) it is also evident that $-\lambda \in \sigma_{p}\left(\mathcal{L}_{\alpha}\right)$.

Finally, by setting $\lambda=0$ in Eq. (4.5), we obtain from Proposition 4.6 and Lemma 4.7 that

$$
\operatorname{ker} \mathcal{L}_{\alpha}=\left\langle\left(\begin{array}{c}
0 \\
Q_{\mathbb{R}^{d}}+\rho_{\alpha}
\end{array}\right)\right\rangle \text {. }
$$

In particular, $0 \in \sigma_{p}\left(\mathcal{L}_{\alpha}\right)$.

Now we can show that the linear stability of the soliton in the curved geometry is determined by the stability of the Euclidean ground state, at least if $p \neq 1+\frac{4}{d}$.

Lemma 4.9. If $p \neq 1+\frac{4}{d}$ then there exists an $\alpha_{0}>0$ such that for all $\alpha \geq \alpha_{0}$ the following holds.

- The algebraic multiplicity of $0 \in \sigma_{p}\left(\mathcal{L}_{\alpha}\right)$ equals 2 .

- If $p<1+\frac{4}{d}$, there are no positive eigenvalues of $\mathcal{L}_{\alpha}$.

- If $p>1+\frac{4}{d}$, there exists precisely one positive eigenvalue $\lambda_{\alpha} \in \sigma_{p}\left(\mathcal{L}_{\alpha}\right)$ and the eigenvalues $\pm \lambda_{\alpha} \in \sigma_{p}\left(\mathcal{L}_{\alpha}\right)$ are simple.

Proof. Acoording to Lemma 4.8, there exists a $\mu>0$ such that $(-\infty,-\mu) \cup(\mu, \infty) \subset \rho\left(\mathcal{L}_{\alpha}\right)$ for all $\alpha \geq \alpha_{0}$. Let $\gamma:[0,1] \rightarrow \mathbb{C}$ be a simple, closed, smooth curve such that $\gamma(t) \in \rho(\mathcal{L})$ for all $t \in[0,1]$ and $\gamma$ encircles the interval $[-\mu-1, \mu+1]$ in such a way that only real eigenvalues of $\mathcal{L}$ lie inside of $\gamma$. This is possible since $0 \in \sigma_{p}(\mathcal{L})$ is isolated. Let

$$
\mathcal{P}:=\frac{1}{2 \pi i} \int_{\gamma}(z-\mathcal{L})^{-1} d z
$$

Since $\mathcal{L}_{\alpha}-\mathcal{L}$ is bounded and converges to 0 in norm as $\alpha \rightarrow \infty$ (see the proofs of Proposition 4.6 and Lemma 4.7), $\gamma(t) \in \rho\left(\mathcal{L}_{\alpha}\right)$ for all $t \in[0,1]$ and all $\alpha \geq \alpha_{0}$, provided $\alpha_{0}>0$ is sufficiently large. Consequently,

$$
\mathcal{P}_{\alpha}:=\frac{1}{2 \pi i} \int_{\gamma}\left(z-\mathcal{L}_{\alpha}\right)^{-1} d z
$$

is well defined, and $\mathcal{P}_{\alpha} \rightarrow \mathcal{P}$ in norm as $\alpha \rightarrow \infty$. This implies

$$
\operatorname{dim} \operatorname{rg} \mathcal{P}_{\alpha}=\operatorname{dim} \operatorname{rg} \mathcal{P}= \begin{cases}2, & p<1+\frac{4}{d} \\ 4, & p>1+\frac{4}{d}\end{cases}
$$


by Theorem 4.3. Suppose now that $p<1+\frac{4}{d}$ and there exists a positive eigenvalue $\lambda_{\alpha} \in \sigma_{p}\left(\mathcal{L}_{\alpha}\right)$. Then, by Lemma 4.8, $-\lambda_{\alpha} \in \sigma_{p}\left(\mathcal{L}_{\alpha}\right)$ and, since $0 \in \sigma_{p}\left(\mathcal{L}_{\alpha}\right)$, we must have $\operatorname{dim} \operatorname{rg} \mathcal{P}_{\alpha} \geq 3$. This contradicts $\operatorname{dim} \operatorname{rg} \mathcal{P}_{\alpha}=2$ and thus, there can be no positive eigenvalue of $\mathcal{L}_{\alpha}$ in the case $p<1+\frac{4}{d}$. If $p>1+\frac{4}{d}$, there exists a unique positive eigenvalue $\lambda \in \sigma_{p}(\mathcal{L})$ with algebraic multiplicity 1 (Theorem 4.3). Let $\widetilde{\gamma}:[0,1] \rightarrow \rho(\mathcal{L}) \subset \mathbb{C}$ be a simple, closed, smooth curve that encircles the interval $\left[\frac{\lambda}{2}, \mu+1\right]$ and such that $\lambda$ is the only spectral point of $\mathcal{L}$ that lies inside of $\widetilde{\gamma}$. Set

$$
\widetilde{\mathcal{P}}:=\frac{1}{2 \pi i} \int_{\widetilde{\gamma}}(z-\mathcal{L})^{-1} d z
$$

As above,

$$
\widetilde{\mathcal{P}}_{\alpha}:=\frac{1}{2 \pi i} \int_{\widetilde{\gamma}}\left(z-\mathcal{L}_{\alpha}\right)^{-1} d z
$$

is well defined for sufficiently large $\alpha$ and $\operatorname{dim} \operatorname{rg} \widetilde{\mathcal{P}}_{\alpha}=\operatorname{dim} \operatorname{rg} \widetilde{\mathcal{P}}=1$. Consequently, there exists a positive simple eigenvalue $\lambda_{\alpha} \in \sigma_{p}\left(\mathcal{L}_{\alpha}\right)$ and by Lemma 4.8, $-\lambda_{\alpha} \in \sigma_{p}\left(\mathcal{L}_{\alpha}\right)$. Furthermore, by symmetry, $-\lambda_{\alpha} \in \sigma_{p}\left(\mathcal{L}_{\alpha}\right)$ must be simple, too. Since $\operatorname{dim} \operatorname{rg} \mathcal{P}_{\alpha}=4$ and $0 \in \sigma_{p}\left(\mathcal{L}_{\alpha}\right)$, there can be no other nonzero eigenvalues in $[-\mu-1, \mu+1]$ as they would have to come in pairs. Since $(\mu, \infty) \subset \rho\left(\mathcal{L}_{\alpha}\right)$, it follows that there exists a unique simple positive eigenvalue $\lambda_{\alpha} \in \sigma_{p}\left(\mathcal{L}_{\alpha}\right)$. In particular, the algebraic multiplicity of $0 \in \sigma_{p}\left(\mathcal{L}_{\alpha}\right)$ must equal 2.

\section{Spectral stability in the CRitical CASE}

In the critical case $p=1+\frac{4}{d}$, the situation is subtle and the stability of the soliton depends on the fine structure of the geometry.

\subsection{Refined properties of $\mathcal{L}_{\alpha,+}$.}

Lemma 5.1. There exists an $\alpha_{0}>0$ such that for all $\alpha \geq \alpha_{0}$ the following holds. We have

$$
\sigma_{e}\left(\mathcal{L}_{\alpha,+}\right)=\left[1+V_{0, d} \alpha^{-2}, \infty\right),
$$

$\mathcal{L}_{\alpha,+}$ has precisely one negative eigenvalue $\lambda_{\alpha}^{*}<0$, and this eigenvalue is simple. Furthermore, if $f_{\alpha}^{*} \in H_{\mathrm{rad}}^{2}\left(\mathbb{R}^{d}\right) \backslash\{0\}$ is an associated eigenfunction, i.e., $\mathcal{L}_{\alpha,+} f_{\alpha}^{*}=\lambda_{\alpha}^{*} f_{\alpha}^{*}$, then we have

$$
\left(f_{\alpha}^{*} \mid Q_{\mathbb{R}^{d}}+\rho_{\alpha}\right)_{L^{2}\left(\mathbb{R}^{d}\right)} \neq 0 .
$$

Proof. As in the proof of Proposition [4.6, we write $\mathcal{L}_{\alpha,+} f=\mathcal{L}_{\alpha, 0} f+W_{\alpha} f$, where $\mathcal{L}_{\alpha, 0}$ : $H_{\text {rad }}^{2}\left(\mathbb{R}^{d}\right) \subset L_{\text {rad }}^{2}\left(\mathbb{R}^{d}\right) \rightarrow L_{\text {rad }}^{2}\left(\mathbb{R}^{d}\right)$ is given by $\mathcal{L}_{\alpha, 0} f=-\Delta_{\mathbb{R}^{d}} f+\left(1+V_{0, d} \alpha^{-2}\right) f$, and

$$
W_{\alpha}(x):=-p \varphi_{d, p}\left(\alpha^{-1} x\right)\left|Q_{\mathbb{R}^{d}}(x)+\rho_{\alpha}(x)\right|^{p-1}+\alpha^{-2}\left[V_{d}\left(\alpha^{-1} x\right)-V_{0, d}\right] .
$$

Thus, by repeating the argument from the proof of Proposition 4.6, we find $\sigma_{e}\left(\mathcal{L}_{\alpha,+}\right)=$ $\left[1+V_{0, d} \alpha^{-2}, \infty\right)$, and there exists a $\mu>0$ such that $(-\infty,-\mu) \subset \rho\left(\mathcal{L}_{\alpha,+}\right)$ for all $\alpha$ sufficiently large.

We define $\mathcal{C}_{\alpha}: L_{\text {rad }}^{2}\left(\mathbb{R}^{d}\right) \rightarrow L_{\text {rad }}^{2}\left(\mathbb{R}^{d}\right)$ by

$$
\begin{aligned}
\mathcal{C}_{\alpha} f(x) & =\mathcal{L}_{\alpha,+} f(x)-\mathcal{L}_{+} f(x) \\
& =\left[-p \varphi_{d, p}\left(\alpha^{-1} x\right)\left|Q_{\mathbb{R}^{d}}(x)+\rho_{\alpha}(x)\right|^{p-1}+p\left|Q_{\mathbb{R}^{d}}(x)\right|^{p-1}+\alpha^{-2} V_{d}\left(\alpha^{-1} x\right)\right] f(x),
\end{aligned}
$$


As in the proof of Proposition 4.6, we infer that $\mathcal{C}_{\alpha} \rightarrow 0$ in norm as $\alpha \rightarrow \infty$. Let $\gamma:[0,1] \rightarrow \mathbb{C}$ be given by $\gamma(t)=-\mu+\mu e^{2 \pi i t}$. Then, by Lemma 4.7 and the above, $\gamma(t) \in \rho\left(\mathcal{L}_{\alpha,+}\right)$ for all $t \in[0,1)$ and all $\alpha \geq \alpha_{0}$, provided $\alpha_{0}>0$ is sufficiently large. Define the spectral projections

$$
\mathcal{P}:=\frac{1}{2 \pi i} \int_{\gamma}\left(z-\mathcal{L}_{+}\right)^{-1} d z, \quad \mathcal{P}_{\alpha}:=\frac{1}{2 \pi i} \int_{\gamma}\left(z-\mathcal{L}_{\alpha,+}\right)^{-1} d z
$$

Then $\mathcal{P}_{\alpha} \rightarrow \mathcal{P}$ in norm as $\alpha \rightarrow \infty$. Furthermore, $\mathcal{L}_{+}$has precisely one simple eigenvalue inside of $\gamma$ [71, 16], which implies that $\operatorname{dim} \operatorname{rg} \mathcal{P}=1$. Consequently, from [43], p. 34, Lemma 4.10, we conclude that $\operatorname{dim} \operatorname{rg} \mathcal{P}_{\alpha}=\operatorname{dim} \operatorname{rg} \mathcal{P}=1$ for all $\alpha \geq \alpha_{0}$. In conjunction with $(-\infty,-\mu) \subset \rho\left(\mathcal{L}_{\alpha,+}\right)$ and the self-adjointness of $\mathcal{L}_{\alpha,+}$ (Lemma 4.7), this means that $\mathcal{L}_{\alpha,+}$ has precisely one negative eigenvalue $\lambda_{\alpha}^{*}<0$, and this eigenvalue is simple.

Let $f^{*} \in \operatorname{rg} \mathcal{P}$ with $\left\|f^{*}\right\|_{L^{2}\left(\mathbb{R}^{d}\right)}=1$ and set $f_{\alpha}^{*}:=\mathcal{P}_{\alpha} f^{*}$. Then $f^{*}$ is an eigenfunction of $\mathcal{L}_{+}$ to the eigenvalue $\lambda^{*}<0$. Furthermore,

$$
\left\|f_{\alpha}^{*}-f^{*}\right\|_{L^{2}\left(\mathbb{R}^{d}\right)}=\left\|\left(\mathcal{P}_{\alpha}-\mathcal{P}\right) f^{*}\right\|_{L^{2}\left(\mathbb{R}^{d}\right)} \rightarrow 0
$$

as $\alpha \rightarrow \infty$ and thus, $f_{\alpha}^{*} \neq 0$ for all $\alpha \geq \alpha_{0}$ if $\alpha_{0}>0$ is sufficiently large. As a consequence, $f_{\alpha}^{*}$ is an eigenfunction of $\mathcal{L}_{\alpha,+}$ with eigenvalue $\lambda_{\alpha}^{*}$ and any other eigenfunction to this eigenvalue is a multiple of $f_{\alpha}^{*}$. Since $\lambda^{*}$ is the only negative eigenvalue of $\mathcal{L}_{+}$, it follows by Sturm oscillation theory that $f^{*}$ does not have zeros. In particular, $\left(f^{*} \mid Q_{\mathbb{R}^{d}}\right)_{L^{2}\left(\mathbb{R}^{d}\right)} \neq 0$. The fact that

$$
\left(f_{\alpha}^{*} \mid Q_{\mathbb{R}^{d}}+\rho_{\alpha}\right)_{L^{2}\left(\mathbb{R}^{d}\right)} \rightarrow\left(f^{*} \mid Q_{\mathbb{R}^{d}}\right)_{L^{2}\left(\mathbb{R}^{d}\right)}
$$

as $\alpha \rightarrow \infty$ thus implies that $\left(f_{\alpha}^{*} \mid Q_{\mathbb{R}^{d}}+\rho_{\alpha}\right)_{L^{2}\left(\mathbb{R}^{d}\right)} \neq 0$ for all $\alpha \geq \alpha_{0}$, provided $\alpha_{0}>0$ is sufficiently large.

Lemma 5.2. Let $\alpha>0$ be sufficiently large and denote by $\mathcal{P}_{\alpha}^{\perp}$ the orthogonal projection onto $\left\langle Q_{\mathbb{R}^{d}}+\rho_{\alpha}\right\rangle^{\perp}$, i.e.,

$$
\mathcal{P}_{\alpha}^{\perp} f:=f-\frac{\left(f \mid Q_{\mathbb{R}^{d}}+\rho_{\alpha}\right)_{L^{2}\left(\mathbb{R}^{d}\right)}}{\left\|Q_{\mathbb{R}^{d}}+\rho_{\alpha}\right\|_{L^{2}\left(\mathbb{R}^{d}\right)}^{2}}\left(Q_{\mathbb{R}^{d}}+\rho_{\alpha}\right) .
$$

Then the operator $\mathcal{P}_{\alpha}^{\perp} \mathcal{L}_{\alpha,+} \mathcal{P}_{\alpha}^{\perp}: H_{\text {rad }}^{2}\left(\mathbb{R}^{d}\right) \subset L_{\text {rad }}^{2}\left(\mathbb{R}^{d}\right) \rightarrow L_{\text {rad }}^{2}\left(\mathbb{R}^{d}\right)$ is self-adjoint, and

$$
\sigma_{e}\left(\mathcal{P}_{\alpha}^{\perp} \mathcal{L}_{\alpha,+} \mathcal{P}_{\alpha}^{\perp}\right)=\left[1+V_{0, d} \alpha^{-2}, \infty\right)
$$

Proof. The proof is based on the standard trick (see e.g. [43], p. 246) of using the decomposition

$$
\mathcal{P}_{\alpha}^{\perp} \mathcal{L}_{\alpha,+} \mathcal{P}_{\alpha}^{\perp}=\mathcal{L}_{\alpha,+}+\left(\mathcal{P}_{\alpha}^{\perp}-1\right) \mathcal{L}_{\alpha,+} \mathcal{P}_{\alpha}^{\perp}+\mathcal{L}_{\alpha,+}\left(\mathcal{P}_{\alpha}^{\perp}-1\right)=: \mathcal{L}_{\alpha,+}+\mathcal{K}_{\alpha}
$$

Since $\operatorname{dim} \operatorname{rg}\left(\mathcal{P}_{\alpha}^{\perp}-1\right)=1$, the operator $\mathcal{K}_{\alpha}: H_{\text {rad }}^{2}\left(\mathbb{R}^{d}\right) \subset L_{\text {rad }}^{2}\left(\mathbb{R}^{d}\right) \rightarrow L_{\text {rad }}^{2}\left(\mathbb{R}^{d}\right)$ has finite rank. The estimate,

$$
\begin{aligned}
\left\|\mathcal{K}_{\alpha} f\right\|_{L^{2}\left(\mathbb{R}^{d}\right)} & \leq \frac{\left|\left(\mathcal{L}_{\alpha,+} \mathcal{P}_{\alpha}^{\perp} f \mid \widetilde{R}_{\alpha}\right)_{L^{2}\left(\mathbb{R}^{d}\right)}\right|}{\left\|\widetilde{R}_{\alpha}\right\|_{L^{2}\left(\mathbb{R}^{d}\right)}^{2}}\left\|\widetilde{R}_{\alpha}\right\|_{L^{2}\left(\mathbb{R}^{d}\right)}+\frac{\left|\left(f \mid \widetilde{R}_{\alpha}\right)_{L^{2}\left(\mathbb{R}^{d}\right)}\right|}{\left\|\widetilde{R}_{\alpha}\right\|_{L^{2}\left(\mathbb{R}^{d}\right)}^{2}}\left\|\mathcal{L}_{\alpha,+} \widetilde{R}_{\alpha}\right\|_{L^{2}\left(\mathbb{R}^{d}\right)} \\
& \leq \frac{\left(f \mid \mathcal{P}_{\alpha}^{\perp} \mathcal{L}_{\alpha,+} \widetilde{R}_{\alpha}\right)_{L^{2}\left(\mathbb{R}^{d}\right)}}{\left\|\widetilde{R}_{\alpha}\right\|_{L^{2}\left(\mathbb{R}^{d}\right)}}+\frac{\left\|\mathcal{L}_{\alpha,+} \widetilde{R}_{\alpha}\right\|_{L^{2}\left(\mathbb{R}^{d}\right)}\|f\|_{L^{2}\left(\mathbb{R}^{d}\right)}}{\left\|\widetilde{R}_{\alpha}\right\|_{L^{2}\left(\mathbb{R}^{d}\right)}} \\
& \lesssim \frac{\left\|\mathcal{L}_{\alpha,+} \widetilde{R}_{\alpha}\right\|_{L^{2}\left(\mathbb{R}^{d}\right)}\|f\|_{L^{2}\left(\mathbb{R}^{d}\right)}}{\left\|\widetilde{R}_{\alpha}\right\|_{L^{2}\left(\mathbb{R}^{d}\right)}}
\end{aligned}
$$


for all $f \in H_{\text {rad }}^{2}\left(\mathbb{R}^{d}\right)$, where $\widetilde{R}_{\alpha}:=Q_{\mathbb{R}^{d}}+\rho_{\alpha}$, shows that $\mathcal{K}_{\alpha}$ extends to a bounded operator $\mathcal{K}_{\alpha}: L_{\text {rad }}^{2}\left(\mathbb{R}^{d}\right) \rightarrow L_{\text {rad }}^{2}\left(\mathbb{R}^{d}\right)$ of finite rank. In particular, $\mathcal{K}_{\alpha}$ is compact. Furthermore,

$$
\mathcal{K}_{\alpha}^{*}=\mathcal{P}_{\alpha}^{\perp} \mathcal{L}_{\alpha,+}\left(\mathcal{P}_{\alpha}^{\perp}-1\right)+\left(\mathcal{P}_{\alpha}^{\perp}-1\right) \mathcal{L}_{\alpha,+}=\mathcal{P}_{\alpha}^{\perp} \mathcal{L}_{\alpha,+} \mathcal{P}_{\alpha}^{\perp}-\mathcal{L}_{\alpha,+}=\mathcal{K}_{\alpha},
$$

and thus $\mathcal{K}_{\alpha}$ is self-adjoint. By the Kato-Rellich theorem (see e.g. [68], p, 159, Theorem 10.2) it follows that $\mathcal{P}_{\alpha}^{\perp} \mathcal{L}_{\alpha,+} \mathcal{P}_{\alpha}^{\perp}$ is self-adjoint. Weyl's theorem (see e.g. [68], p. 171, Theorem 6.19), in conjunction with Lemma 5.1, yields the statement on the essential spectrum.

Next, we establish a crucial dichotomy for $\mathcal{L}_{\alpha,+}$.

Proposition 5.3. Let $\alpha>0$ be sufficiently large.

- If $\left(\mathcal{L}_{\alpha,+}^{-1}\left(Q_{\mathbb{R}^{d}}+\rho_{\alpha}\right) \mid Q_{\mathbb{R}^{d}}+\rho_{\alpha}\right)_{L^{2}\left(\mathbb{R}^{d}\right)}>0$ then there exists an $f_{\alpha} \in\left\langle Q_{\mathbb{R}^{d}}+\rho_{\alpha}\right\rangle^{\perp} \cap H_{\text {rad }}^{2}\left(\mathbb{R}^{d}\right)$ such that

$$
\left(\mathcal{L}_{\alpha,+} f_{\alpha} \mid f_{\alpha}\right)_{L^{2}\left(\mathbb{R}^{d}\right)}<0 .
$$

- If $\left(\mathcal{L}_{\alpha,+}^{-1}\left(Q_{\mathbb{R}^{d}}+\rho_{\alpha}\right) \mid Q_{\mathbb{R}^{d}}+\rho_{\alpha}\right)_{L^{2}\left(\mathbb{R}^{d}\right)} \leq 0$ then

$$
\left(\mathcal{L}_{\alpha,+} f \mid f\right)_{L^{2}\left(\mathbb{R}^{d}\right)} \geq 0
$$

for all $f \in\left\langle Q_{\mathbb{R}^{d}}+\rho_{\alpha}\right\rangle^{\perp} \cap H_{\mathrm{rad}}^{2}\left(\mathbb{R}^{d}\right)$.

Proof. We first assume that

$$
\left(\mathcal{L}_{\alpha,+}^{-1} \widetilde{R}_{\alpha} \mid \widetilde{R}_{\alpha}\right)_{L^{2}\left(\mathbb{R}^{d}\right)}>0
$$

where $\widetilde{R}_{\alpha}=Q_{\mathbb{R}^{d}}+\rho_{\alpha}$. Let $\mathcal{P}_{\alpha}^{\perp}$ be the orthogonal projection on $\left\langle\widetilde{R}_{\alpha}\right\rangle^{\perp}$, i.e.,

$$
\mathcal{P}_{\alpha}^{\perp} f=f-\frac{\left(f \mid \widetilde{R}_{\alpha}\right)_{L^{2}\left(\mathbb{R}^{d}\right)}}{\left\|\widetilde{R}_{\alpha}\right\|_{L^{2}\left(\mathbb{R}^{d}\right)}^{2}} \widetilde{R}_{\alpha} .
$$

We set $f_{\alpha}:=\mathcal{P}_{\alpha}^{\perp} \mathcal{L}_{\alpha,+}^{-1} \widetilde{R}_{\alpha} \in\left\langle\widetilde{R}_{\alpha}\right\rangle^{\perp} \cap H_{\text {rad }}^{2}\left(\mathbb{R}^{d}\right)$. Then we have

$$
\begin{aligned}
\left(\mathcal{L}_{\alpha,+} f_{\alpha} \mid f_{\alpha}\right)_{L^{2}\left(\mathbb{R}^{d}\right)}= & \left(\mathcal{L}_{\alpha,+} \mathcal{P}_{\alpha}^{\perp} \mathcal{L}_{\alpha,+}^{-1} \widetilde{R}_{\alpha} \mid \mathcal{P}_{\alpha}^{\perp} \mathcal{L}_{\alpha,+}^{-1} \widetilde{R}_{\alpha}\right)_{L^{2}\left(\mathbb{R}^{d}\right)} \\
= & \left(\widetilde{R}_{\alpha} \mid \mathcal{L}_{\alpha,+}^{-1} \widetilde{R}_{\alpha}\right)_{L^{2}\left(\mathbb{R}^{d}\right)}-\frac{\left(\mathcal{L}_{\alpha,+}^{-1} \widetilde{R}_{\alpha} \mid \widetilde{R}_{\alpha}\right)_{L^{2}\left(\mathbb{R}^{d}\right)}}{\left\|\widetilde{R}_{\alpha}\right\|_{L^{2}\left(\mathbb{R}^{d}\right)}^{2}}\left(\mathcal{L}_{\alpha,+} \widetilde{R}_{\alpha} \mid \mathcal{L}_{\alpha,+}^{-1} \widetilde{R}_{\alpha}\right)_{L^{2}\left(\mathbb{R}^{d}\right)} \\
& -\frac{\left(\mathcal{L}_{\alpha,+}^{-1} \widetilde{R}_{\alpha} \mid \widetilde{R}_{\alpha}\right)_{L^{2}\left(\mathbb{R}^{d}\right)}}{\left\|\widetilde{R}_{\alpha}\right\|_{L^{2}\left(\mathbb{R}^{d}\right)}^{2}}\left(\widetilde{R}_{\alpha} \mid \widetilde{R}_{\alpha}\right)_{L^{2}\left(\mathbb{R}^{d}\right)} \\
& +\frac{\left(\mathcal{L}_{\alpha,+}^{-1} \widetilde{R}_{\alpha} \mid \widetilde{R}_{\alpha}\right)_{L^{2}\left(\mathbb{R}^{d}\right)}^{2}}{\left\|\widetilde{R}_{\alpha}\right\|_{L^{2}\left(\mathbb{R}^{d}\right)}^{4}}\left(\mathcal{L}_{\alpha,+} \widetilde{R}_{\alpha} \mid \widetilde{R}_{\alpha}\right)_{L^{2}\left(\mathbb{R}^{d}\right)} \\
= & -\left(\mathcal{L}_{\alpha,+}^{-1} \widetilde{R}_{\alpha} \mid \widetilde{R}_{\alpha}\right)_{L^{2}\left(\mathbb{R}^{d}\right)}+\frac{\left(\mathcal{L}_{\alpha,+}^{-1} \widetilde{R}_{\alpha} \mid \widetilde{R}_{\alpha}\right)_{L^{2}\left(\mathbb{R}^{d}\right)}^{2}}{\left\|\widetilde{R}_{\alpha}\right\|_{L^{2}\left(\mathbb{R}^{d}\right)}^{4}}\left(\mathcal{L}_{\alpha,+} \widetilde{R}_{\alpha} \mid \widetilde{R}_{\alpha}\right)_{L^{2}\left(\mathbb{R}^{d}\right)} \\
& <\frac{\left(\mathcal{L}_{\alpha,+}^{-1} \widetilde{R}_{\alpha} \mid \widetilde{R}_{\alpha}\right)_{L^{2}\left(\mathbb{R}^{d}\right)}^{2}\left(\mathcal{L}_{\alpha,+} \widetilde{R}_{\alpha} \mid \widetilde{R}_{\alpha}\right)_{L^{2}\left(\mathbb{R}^{d}\right)}}{\left\|\widetilde{R}_{\alpha}\right\|_{L^{2}\left(\mathbb{R}^{d}\right)}^{4}}
\end{aligned}
$$


Thus, it suffices to show that $\left(\mathcal{L}_{\alpha,+} \widetilde{R}_{\alpha} \mid \widetilde{R}_{\alpha}\right)_{L^{2}\left(\mathbb{R}^{d}\right)} \leq 0$. Explicitly, we have

$$
\begin{aligned}
\mathcal{L}_{\alpha,+} \widetilde{R}_{\alpha}(x) & =\mathcal{L}_{\alpha,-} \widetilde{R}_{\alpha}(x)-(p-1) \varphi_{d, p}\left(\alpha^{-1} x\right)\left|\widetilde{R}_{\alpha}(x)\right|^{p-1} \widetilde{R}_{\alpha}(x) \\
& =-(p-1) \varphi_{d, p}\left(\alpha^{-1} x\right)\left|\widetilde{R}_{\alpha}(x)\right|^{p-1} \widetilde{R}_{\alpha}(x),
\end{aligned}
$$

and thus,

$$
\left(\mathcal{L}_{\alpha,+} \widetilde{R}_{\alpha} \mid \widetilde{R}_{\alpha}\right)_{L^{2}\left(\mathbb{R}^{d}\right)}=-(p-1) \int_{\mathbb{R}^{d}} \varphi_{d, p}\left(\alpha^{-1} x\right)\left|\widetilde{R}_{\alpha}(x)\right|^{p+1} d x<0,
$$

since $\varphi_{d, p} \geq 0$ by Hypothesis 1.1. In summary, $\left(\mathcal{L}_{\alpha,+} f_{\alpha} \mid f_{\alpha}\right)_{L^{2}\left(\mathbb{R}^{d}\right)}<0$, as claimed.

Next, we assume that

$$
\left(\mathcal{L}_{\alpha,+}^{-1} \widetilde{R}_{\alpha} \mid \widetilde{R}_{\alpha}\right)_{L^{2}\left(\mathbb{R}^{d}\right)} \leq 0
$$

Suppose there exists an $f_{\alpha} \in\left\langle\widetilde{R}_{\alpha}\right\rangle^{\perp} \cap H_{\text {rad }}^{2}\left(\mathbb{R}^{d}\right)$ such that $\left(\mathcal{L}_{\alpha,+} f_{\alpha} \mid f_{\alpha}\right)_{L^{2}\left(\mathbb{R}^{d}\right)}<0$. Consider the operator $\mathcal{P}_{\alpha}^{\perp} \mathcal{L}_{\alpha,+} \mathcal{P}_{\alpha}^{\perp}$. By assumption, we have

$$
0>\left(\mathcal{L}_{\alpha,+} f_{\alpha} \mid f_{\alpha}\right)_{L^{2}\left(\mathbb{R}^{d}\right)}=\left(\mathcal{L}_{\alpha,+} \mathcal{P}_{\alpha}^{\perp} f_{\alpha} \mid \mathcal{P}_{\alpha}^{\perp} f_{\alpha}\right)_{L^{2}\left(\mathbb{R}^{d}\right)}=\left(\mathcal{P}_{\alpha}^{\perp} \mathcal{L}_{\alpha,+} \mathcal{P}_{\alpha}^{\perp} f_{\alpha} \mid f_{\alpha}\right)_{L^{2}\left(\mathbb{R}^{d}\right)},
$$

and thus, by Lemma 5.2, $\mathcal{P}_{\alpha}^{\perp} \mathcal{L}_{\alpha,+} \mathcal{P}_{\alpha}^{\perp}$ must have a negative eigenvalue $\lambda_{\alpha}<0$. In other words, there exists a nontrivial $g_{\alpha} \in\left\langle\widetilde{R}_{\alpha}\right\rangle^{\perp} \cap H_{\text {rad }}^{2}\left(\mathbb{R}^{d}\right)$ such that $\mathcal{P}_{\alpha}^{\perp} \mathcal{L}_{\alpha,+} g_{\alpha}=\lambda_{\alpha} g_{\alpha}$. This means that there exists a $c_{\alpha} \in \mathbb{C}$ such that

$$
\mathcal{L}_{\alpha,+} g_{\alpha}=\lambda_{\alpha} g_{\alpha}+c_{\alpha} \widetilde{R}_{\alpha}
$$

We claim that $c_{\alpha} \neq 0$. To see this, recall that $\mathcal{L}_{\alpha,+}$ has a unique negative eigenvalue $\lambda_{\alpha}^{*}<0$ (which is simple) and if $f_{\alpha}^{*}$ is an associated eigenfunction, we have $\left(f_{\alpha}^{*} \mid \widetilde{R}_{\alpha}\right)_{L^{2}\left(\mathbb{R}^{d}\right)} \neq 0$, see Lemma 5.1. Suppose now that $c_{\alpha}=0$. Then $g_{\alpha}$ is an eigenfunction of $\mathcal{L}_{\alpha,+}$ with negative eigenvalue $\lambda_{\alpha}$, and thus, $\lambda_{\alpha}=\lambda_{\alpha}^{*}$ and $g_{\alpha}$ must be a multiple of $f_{\alpha}^{*}$. This, however, contradicts $\left(g_{\alpha} \mid \widetilde{R}_{\alpha}\right)_{L^{2}\left(\mathbb{R}^{d}\right)}=0$, and the claim $c_{\alpha} \neq 0$ follows. Note further that $\lambda_{\alpha} \neq \lambda_{\alpha}^{*}$ because otherwise we would arrive at the contradiction

$$
0=\left(g_{\alpha} \mid\left(\mathcal{L}_{\alpha,+}-\lambda_{\alpha}^{*}\right) f_{\alpha}^{*}\right)_{L^{2}\left(\mathbb{R}^{d}\right)}=\left(\left(\mathcal{L}_{\alpha,+}-\lambda_{\alpha}^{*}\right) g_{\alpha} \mid f_{\alpha}^{*}\right)_{L^{2}\left(\mathbb{R}^{d}\right)}=c_{\alpha}\left(\widetilde{R}_{\alpha} \mid f_{\alpha}^{*}\right)_{L^{2}\left(\mathbb{R}^{d}\right)} \neq 0 .
$$

Consequently, we have

$$
\left(\mathcal{L}_{\alpha,+}-\lambda_{\alpha}\right)^{-1} \widetilde{R}_{\alpha}=\frac{1}{c_{\alpha}} g_{\alpha}
$$

Furthermore,

$$
\left(\left(\mathcal{L}_{\alpha,+}-\lambda_{\alpha}\right)^{-1} \widetilde{R}_{\alpha} \mid \widetilde{R}_{\alpha}\right)_{L^{2}\left(\mathbb{R}^{d}\right)}=\frac{1}{c_{\alpha}}\left(g_{\alpha} \mid \widetilde{R}_{\alpha}\right)_{L^{2}\left(\mathbb{R}^{d}\right)}=0 .
$$

Now we define a function $\phi_{\alpha}:(-\infty, 0] \backslash\left\{\lambda_{\alpha}^{*}\right\} \rightarrow \mathbb{R}$ by

$$
\phi_{\alpha}(\lambda):=\left(\left(\mathcal{L}_{\alpha,+}-\lambda\right)^{-1} \widetilde{R}_{\alpha} \mid \widetilde{R}_{\alpha}\right)_{L^{2}\left(\mathbb{R}^{d}\right)} .
$$

Note that $\phi_{\alpha}$ is differentiable and

$$
\begin{aligned}
\phi_{\alpha}^{\prime}(\lambda) & =\left(\left(\mathcal{L}_{\alpha,+}-\lambda\right)^{-2} \widetilde{R}_{\alpha} \mid \widetilde{R}_{\alpha}\right)_{L^{2}\left(\mathbb{R}^{d}\right)}=\left(\left(\mathcal{L}_{\alpha,+}-\lambda\right)^{-1} \widetilde{R}_{\alpha} \mid\left(\mathcal{L}_{\alpha,+}-\lambda\right)^{-1} \widetilde{R}_{\alpha}\right)_{L^{2}\left(\mathbb{R}^{d}\right)} \\
& =\left\|\left(\mathcal{L}_{\alpha,+}-\lambda\right)^{-1} \widetilde{R}_{\alpha}\right\|_{L^{2}\left(\mathbb{R}^{d}\right)}^{2}>0
\end{aligned}
$$


for all $\lambda \in(-\infty, 0] \backslash\left\{\lambda_{\alpha}^{*}\right\}$. By assumption,

$$
\phi_{\alpha}(0)=\left(\mathcal{L}_{\alpha,+}^{-1} \widetilde{R}_{\alpha} \mid \widetilde{R}_{\alpha}\right)_{L^{2}\left(\mathbb{R}^{d}\right)} \leq 0
$$

and $\phi_{\alpha}\left(\lambda_{\alpha}\right)=0$. Thus, we must have $\lambda_{\alpha}<\lambda_{\alpha}^{*}$ since otherwise, we would arrive at the contradiction

$$
0 \geq \phi_{\alpha}(0)=\int_{\lambda_{\alpha}}^{0} \phi_{\alpha}^{\prime}(\lambda) d \lambda+\underbrace{\phi_{\alpha}\left(\lambda_{\alpha}\right)}_{=0}>0 .
$$

However, $\lambda_{\alpha}<\lambda_{\alpha}^{*}$ is also impossible since it leads to the contradiction

$$
\begin{aligned}
0 & =\phi_{\alpha}\left(\lambda_{\alpha}\right)=\left(\left(\mathcal{L}_{\alpha,+}-\lambda_{\alpha}\right)^{-1} \widetilde{R}_{\alpha} \mid \widetilde{R}_{\alpha}\right)_{L^{2}\left(\mathbb{R}^{d}\right)}=\left(\left(\mathcal{L}_{\alpha,+}-\lambda_{\alpha}\right) \widetilde{S}_{\alpha} \mid \widetilde{S}_{\alpha}\right)_{L^{2}\left(\mathbb{R}^{d}\right)} \\
& =\left(\left(\mathcal{L}_{\alpha,+}-\lambda_{\alpha}^{*}\right) \widetilde{S}_{\alpha} \mid \widetilde{S}_{\alpha}\right)_{L^{2}\left(\mathbb{R}^{d}\right)}+\left(\lambda_{\alpha}^{*}-\lambda_{\alpha}\right)\left\|\widetilde{S}_{\alpha}\right\|_{L^{2}\left(\mathbb{R}^{d}\right)}^{2} \\
& >0
\end{aligned}
$$

where $\widetilde{S}_{\alpha}:=\left(\mathcal{L}_{\alpha,+}-\lambda_{\alpha}\right)^{-1} \widetilde{R}_{\alpha}$ and we have used the fact that $\mathcal{L}_{\alpha,+}-\lambda_{\alpha}^{*}$ is nonnegative, see Lemma 5.1. In summary, we see that there cannot exist an $f_{\alpha} \in\left\langle\widetilde{R}_{\alpha}\right\rangle^{\perp} \cap H_{\text {rad }}^{2}\left(\mathbb{R}^{d}\right)$ with $\left(\mathcal{L}_{\alpha,+} f_{\alpha} \mid f_{\alpha}\right)_{L^{2}\left(\mathbb{R}^{d}\right)}<0$, and this finishes the proof.

5.2. The auxiliary operator $\mathcal{L}_{\alpha,-}^{\frac{1}{2}} \mathcal{L}_{\alpha,+} \mathcal{L}_{\alpha,-}^{\frac{1}{2}}$. By Proposition 4.6, $\mathcal{L}_{\alpha,-}$ is nonnegative and thus, the square root $\mathcal{L}_{\alpha,-}^{\frac{1}{2}}$ is well defined either via the functional calculus for self-adjoint operators or by the Dunford-Taylor integral, see e.g. [43], p. 281, Theorem 3.35. Furthermore, since $\mathcal{L}_{\alpha,-}^{\frac{1}{2}}$ is self-adjoint, we have

$$
\operatorname{ker} \mathcal{L}_{\alpha,-}^{\frac{1}{2}}=\operatorname{ker} \mathcal{L}_{\alpha,-}=\left\langle Q_{\mathbb{R}^{d}}+\rho_{\alpha}\right\rangle
$$

and $\operatorname{rg} \mathcal{L}_{\alpha,-}^{\frac{1}{2}}=\left\langle Q_{\mathbb{R}^{d}}+\rho_{\alpha}\right\rangle^{\perp}$ by Proposition 4.6. As expected from the Euclidean case, the auxiliary operator $\mathcal{L}_{\alpha,-}^{\frac{1}{2}} \mathcal{L}_{\alpha,+} \mathcal{L}_{\alpha,-}^{\frac{1}{2}}$ plays a crucial role.

Definition 5.4. Let $\alpha>0$ be sufficiently large. We define an operator

$$
\mathcal{J}_{\alpha}: \mathcal{D}\left(\mathcal{J}_{\alpha}\right) \subset\left\langle Q_{\mathbb{R}^{d}}+\rho_{\alpha}\right\rangle^{\perp} \rightarrow\left\langle Q_{\mathbb{R}^{d}}+\rho_{\alpha}\right\rangle^{\perp}
$$

by

$$
\mathcal{D}\left(\mathcal{J}_{\alpha}\right):=\left\{f \in \mathcal{D}\left(\mathcal{L}_{\alpha,-}^{\frac{1}{2}}\right) \cap\left\langle Q_{\mathbb{R}^{d}}+\rho_{\alpha}\right\rangle^{\perp}: \mathcal{L}_{\alpha,-}^{\frac{1}{2}} f \in \mathcal{D}\left(\mathcal{L}_{\alpha,+}\right) \text { and } \mathcal{L}_{\alpha,+} \mathcal{L}_{\alpha,-}^{\frac{1}{2}} f \in \mathcal{D}\left(\mathcal{L}_{\alpha,-}^{\frac{1}{2}}\right)\right\}
$$

and $\mathcal{J}_{\alpha} f:=\mathcal{L}_{\alpha,-}^{\frac{1}{2}} \mathcal{L}_{\alpha,+} \mathcal{L}_{\alpha,-}^{\frac{1}{2}} f$.

It is not immediately obvious that $\mathcal{J}_{\alpha}$ is densely defined. Thus, we first establish this fact using the following simple property of maximally defined products.

Lemma 5.5. Let $\left(X, \|\left.\cdot\right|_{X}\right),\left(Y,\|\cdot\|_{Y}\right)$, and $\left(Z,\|\cdot\|_{Z}\right)$ be Banach spaces. Furthermore, let $A: \mathcal{D}(A) \subset Y \rightarrow Z$ and $B: \mathcal{D}(B) \subset X \rightarrow Y$ be densely defined linear operators and assume that $B$ is bounded invertible. Then the maximally defined operatol $A B: \mathcal{D}(A B) \subset X \rightarrow Z$ is densely defined and $\mathcal{D}(A B)$ is a core for $B$.

\footnotetext{
${ }^{2}$ That is to say, $\mathcal{D}(A B):=\{x \in \mathcal{D}(B): B x \in \mathcal{D}(A)\}$.
} 
Proof. Let $x \in X$ and $\epsilon>0$ be arbitrary. Since $\mathcal{D}(B)$ is dense in $X$, we can find an $x^{\prime} \in \mathcal{D}(B)$ such that $\left\|x-x^{\prime}\right\|_{X}<\frac{\epsilon}{2}$. By the density of $\mathcal{D}(A)$ in $Y$, there exists a $\widetilde{y} \in \mathcal{D}(A)$ such that $\left\|B x^{\prime}-\widetilde{y}\right\|_{Y}<\frac{\epsilon}{2}\left\|B^{-1}\right\|_{\mathcal{B}(X, Y)}^{-1}$. Set $\widetilde{x}:=B^{-1} \widetilde{y}$. By definition, $\widetilde{x} \in \mathcal{D}(A B)$ and

$$
\begin{aligned}
\|x-\widetilde{x}\|_{X} & =\left\|x-x^{\prime}\right\|_{X}+\left\|x^{\prime}-\widetilde{x}\right\|_{X}<\frac{\epsilon}{2}+\left\|B^{-1}\left(B x^{\prime}-B \widetilde{x}\right)\right\|_{X} \\
& \leq \frac{\epsilon}{2}+\left\|B^{-1}\right\|_{\mathcal{B}(X, Y)}\left\|B x^{\prime}-\widetilde{y}\right\|_{Y}<\epsilon .
\end{aligned}
$$

To prove the second assertion, let $x \in \mathcal{D}(B)$. We have to show that there exists a sequence $\left(x_{n}\right)_{n \in \mathbb{N}} \subset \mathcal{D}(A B)$ such that $x_{n} \rightarrow x$ in $X$ and $B x_{n} \rightarrow B x$ in $Y$ as $n \rightarrow \infty$. Since $\mathcal{D}(A)$ is dense in $Y$, there exists a sequence $\left(y_{n}\right)_{n \in \mathbb{N}} \subset \mathcal{D}(A)$ such that $y_{n} \rightarrow B x$ in $Y$ as $n \rightarrow \infty$. We set $x_{n}:=B^{-1} y_{n}$. Then $\left(x_{n}\right)_{n \in \mathbb{N}} \subset \mathcal{D}(A B)$ and we have $B x_{n} \rightarrow B x$ in $Y$ as well as

$$
\left\|x_{n}-x\right\|_{X}=\left\|B^{-1}\left(B x_{n}-B x\right)\right\|_{X} \lesssim\left\|y_{n}-B x\right\|_{Y} \rightarrow 0
$$

as $n \rightarrow \infty$.

Lemma 5.6. Let $\alpha>0$ be sufficiently large. Then the operator $\mathcal{J}_{\alpha}$ is densely defined.

Proof. To begin with, we define an auxiliary operator $\mathcal{A}_{\alpha}: \mathcal{D}\left(\mathcal{A}_{\alpha}\right) \subset L_{\mathrm{rad}}^{2}\left(\mathbb{R}^{d}\right) \rightarrow\left\langle Q_{\mathbb{R}^{d}}+\rho_{\alpha}\right\rangle^{\perp}$ by

$$
\mathcal{D}\left(\mathcal{A}_{\alpha}\right):=\left\{f \in \mathcal{D}\left(\mathcal{L}_{\alpha,+}\right)=H_{\mathrm{rad}}^{2}\left(\mathbb{R}^{d}\right): \mathcal{L}_{\alpha,+} f \in \mathcal{D}\left(\mathcal{L}_{\alpha,-}^{\frac{1}{2}}\right)\right\}
$$

and $\mathcal{A}_{\alpha} f:=\mathcal{L}_{\alpha,-}^{\frac{1}{2}} \mathcal{L}_{\alpha,+} f$. Since $0 \notin \sigma\left(\mathcal{L}_{\alpha,+}\right)$ by Lemma 4.7. Lemma 5.5 shows that $\mathcal{A}_{\alpha}$ is densely defined. Next, we define another auxiliary operator $\mathcal{B}_{\alpha}: \mathcal{D}\left(\mathcal{B}_{\alpha}\right) \subset\left\langle Q_{\mathbb{R}^{d}}+\rho_{\alpha}\right\rangle^{\perp} \rightarrow$ $\left\langle Q_{\mathbb{R}^{d}}+\rho_{\alpha}\right\rangle^{\perp}$ by

$$
\mathcal{D}\left(\mathcal{B}_{\alpha}\right):=\mathcal{D}\left(\mathcal{L}_{\alpha,-}^{\frac{1}{2}}\right) \cap\left\langle Q_{\mathbb{R}^{d}}+\rho_{\alpha}\right\rangle^{\perp}
$$

and $\mathcal{B}_{\alpha} f:=\mathcal{L}_{\alpha,-}^{\frac{1}{2}} f$. Obviously, $\mathcal{B}_{\alpha}$ is densely defined and, since ker $\mathcal{L}_{\alpha,-}^{\frac{1}{2}}=\left\langle Q_{\mathbb{R}^{d}}+\rho_{\alpha}\right\rangle$, it follows that $\mathcal{B}_{\alpha}$ is injective. Furthermore, by the self-adjointness of $\mathcal{L}_{\alpha,-}^{\frac{1}{2}}, \operatorname{rg} \mathcal{L}_{\alpha,-}^{\frac{1}{2}}=\left\langle Q_{\mathbb{R}^{d}}+\right.$ $\left.\rho_{\alpha}\right\rangle^{\perp}$ and thus, for any $g \in\left\langle Q_{\mathbb{R}^{d}}+\rho_{\alpha}\right\rangle^{\perp}$ we can find an $f \in \mathcal{D}\left(\mathcal{L}_{\alpha,-}^{\frac{1}{2}}\right)$ such that $\mathcal{L}_{\alpha,-}^{\frac{1}{2}} \mathcal{P}_{\alpha}^{\perp} f=$ $\mathcal{L}_{\alpha,-}^{\frac{1}{2}} f=g$, where $\mathcal{P}_{\alpha}^{\perp}$ denotes the orthogonal projection on $\left\langle Q_{\mathbb{R}^{d}}+\rho_{\alpha}\right\rangle^{\perp}$. Consequently, $\mathcal{P}_{\alpha}^{\perp} f \in \mathcal{D}\left(\mathcal{B}_{\alpha}\right)$ and $\mathcal{B}_{\alpha} \mathcal{P}_{\alpha}^{\perp} f=g$. This shows that $\mathcal{B}_{\alpha}$ is surjective. From the closedness of $\mathcal{L}_{\alpha,-}^{\frac{1}{2}}$ it follows immediately that $\mathcal{B}_{\alpha}$ is closed and the closed graph theorem implies that $\mathcal{B}_{\alpha}$ is bounded invertible. Now observe that $\mathcal{J}_{\alpha}=\mathcal{A}_{\alpha} \mathcal{B}_{\alpha}$, where the product $\mathcal{A}_{\alpha} \mathcal{B}_{\alpha}$ is maximally defined. Consequently, Lemma 5.5 implies that $\mathcal{J}_{\alpha}$ is densely defined.

Remark 5.7. Lemma 5.5 also shows that $\mathcal{D}\left(\mathcal{J}_{\alpha}\right)$ is a core for the operator $\mathcal{B}_{\alpha}$ defined in the proof of Lemma 5.6.

The importance of $\mathcal{J}_{\alpha}$ derives from the following observation.

Lemma 5.8. Let $\alpha>0$ be sufficiently large and $\lambda \in \mathbb{C} \backslash\{0\}$. Then we have the following implications.

- If $\lambda \in \rho\left(\mathcal{L}_{\alpha}\right)$ then $\lambda^{2}+\mathcal{J}_{\alpha}$ is surjective.

- The operator $\lambda-\mathcal{L}_{\alpha}$ is injective if and only if $\lambda^{2}+\mathcal{J}_{\alpha}$ is injective. 
Proof. Let $\lambda \in \rho\left(\mathcal{L}_{\alpha}\right)$ and $g \in\left\langle Q_{\mathbb{R}^{d}}+\rho_{\alpha}\right\rangle^{\perp}$. We have to show that there exists an $f \in \mathcal{D}\left(\mathcal{J}_{\alpha}\right)$ such that $\left(\lambda^{2}+\mathcal{J}_{\alpha}\right) f=g$. By the self-adjointness of $\mathcal{L}_{\alpha,-}^{\frac{1}{2}}$, we have

$$
\operatorname{rg} \mathcal{L}_{\alpha,-}^{\frac{1}{2}}=\left(\operatorname{ker} \mathcal{L}_{\alpha,-}^{\frac{1}{2}}\right)^{\perp}
$$

and thus, there exists an $g_{2} \in \mathcal{D}\left(\mathcal{L}_{\alpha,-}^{\frac{1}{2}}\right)$ such that $\lambda \mathcal{L}_{\alpha,-}^{\frac{1}{2}} g_{2}=g$. Since $\lambda \in \rho\left(\mathcal{L}_{\alpha}\right)$, there exists $\left(f_{1}, f_{2}\right) \in H^{2}\left(\mathbb{R}^{d}, \mathbb{C}^{2}\right)$ such that

$$
\left(\lambda-\mathcal{L}_{\alpha}\right)\left(\begin{array}{c}
f_{1} \\
f_{2}
\end{array}\right)=\left(\begin{array}{c}
0 \\
g_{2}
\end{array}\right)
$$

Equivalently,

$$
\left\{\begin{array}{l}
\lambda f_{1}-\mathcal{L}_{\alpha,-} f_{2}=0 \\
\mathcal{L}_{\alpha,+} f_{1}+\lambda f_{2}=g_{2}
\end{array}\right.
$$

By inserting the first equation into the second one, we find

$$
\mathcal{L}_{\alpha,+} \mathcal{L}_{\alpha,-} f_{2}=-\lambda^{2} f_{2}+\lambda g_{2} \in \mathcal{D}\left(\mathcal{L}_{\alpha,-}^{\frac{1}{2}}\right),
$$

and applying $\mathcal{L}_{\alpha,-}^{\frac{1}{2}}$ yields

$$
\mathcal{L}_{\alpha,-}^{\frac{1}{2}} \mathcal{L}_{\alpha,+} \mathcal{L}_{\alpha,-}^{\frac{1}{2}} f=-\lambda^{2} f+\lambda \mathcal{L}_{\alpha,-}^{\frac{1}{2}} g_{2}=-\lambda^{2} f+g
$$

with $f:=\mathcal{L}_{\alpha,-}^{\frac{1}{2}} f_{2} \in \mathcal{D}\left(\mathcal{J}_{\alpha}\right)$.

To prove the second assertion, we first assume that $\lambda^{2}+\mathcal{J}_{\alpha}$ is injective. Suppose

$$
\left(\lambda-\mathcal{L}_{\alpha}\right)\left(\begin{array}{l}
f_{1} \\
f_{2}
\end{array}\right)=\left(\begin{array}{l}
0 \\
0
\end{array}\right) .
$$

Then, by setting $g=g_{2}=0$ in the above computation, we find $\left(\lambda^{2}+\mathcal{J}_{\alpha}\right) f=0$ for $f=\mathcal{L}_{\alpha,-}^{\frac{1}{2}} f_{2}$. This shows that $f_{2} \in \operatorname{ker} \mathcal{L}_{\alpha,-}^{\frac{1}{2}}=\operatorname{ker} \mathcal{L}_{\alpha,-}$ and the first equation in (5.1) implies that $f_{1}=0$. Subsequently, the second equation in (5.1) with $g_{2}=0$ shows that $f_{2}=0$ as well.

It remains to prove the reverse implication, i.e., we assume that $\lambda-\mathcal{L}_{\alpha}$ is injective and show that $\lambda^{2}+\mathcal{J}_{\alpha}$ is injective. Consider the equation $\left(\lambda^{2}+\mathcal{J}_{\alpha}\right) f=0$ for an arbitrary $f \in \mathcal{D}\left(\mathcal{J}_{\alpha}\right)$. We have to show that $f=0$. Set $f_{1}:=\mathcal{L}_{\alpha,-}^{\frac{1}{2}} f$. From $f \in \mathcal{D}\left(\mathcal{J}_{\alpha}\right)$ we infer that $f_{1} \in \mathcal{D}\left(\mathcal{L}_{\alpha,-}^{\frac{1}{2}} \mathcal{L}_{\alpha,+}\right) \subset \mathcal{D}\left(\mathcal{L}_{\alpha,+}\right)=H_{\text {rad }}^{2}\left(\mathbb{R}^{d}\right)$. Furthermore, we define $f_{2}:=-\frac{1}{\lambda} \mathcal{L}_{\alpha,+} f_{1}=$ $-\frac{1}{\lambda} \mathcal{L}_{\alpha,+} \mathcal{L}_{\alpha,-}^{\frac{1}{2}} f \in \mathcal{D}\left(\mathcal{L}_{\alpha,-}^{\frac{1}{2}}\right)$. Then we have

$$
\mathcal{L}_{\alpha,-}^{\frac{1}{2}} f_{2}=-\frac{1}{\lambda} \mathcal{J}_{\alpha} f=\lambda f \in \mathcal{D}\left(\mathcal{J}_{\alpha}\right) \subset \mathcal{D}\left(\mathcal{L}_{\alpha,-}^{\frac{1}{2}}\right),
$$

which shows that $f_{2} \in \mathcal{D}\left(\mathcal{L}_{\alpha,-}\right)=H_{\text {rad }}^{2}\left(\mathbb{R}^{d}\right)$. Consequently,

$$
\left(\lambda-\mathcal{L}_{\alpha}\right)\left(\begin{array}{l}
f_{1} \\
f_{2}
\end{array}\right)=\left(\begin{array}{c}
\lambda f_{1}-\mathcal{L}_{\alpha,-} f_{2} \\
\mathcal{L}_{\alpha,+} f_{1}+\lambda f_{2}
\end{array}\right)=\left(\begin{array}{c}
\lambda \mathcal{L}_{\alpha,-}^{\frac{1}{2}} f-\lambda \mathcal{L}_{\alpha,-}^{\frac{1}{2}} f \\
0
\end{array}\right)=\left(\begin{array}{l}
0 \\
0
\end{array}\right)
$$

and it follows that $f_{1}=f_{2}=0$ by the injectivity of $\lambda-\mathcal{L}_{\alpha}$. Since $f_{1}=\mathcal{L}_{\alpha,-}^{\frac{1}{2}} f$, we infer that $f \in \operatorname{ker} \mathcal{L}_{\alpha,-}^{\frac{1}{2}}=\left\langle Q_{\mathbb{R}^{d}}+\rho_{\alpha}\right\rangle$. Together with $f \in \mathcal{D}\left(\mathcal{J}_{\alpha}\right)$, this implies that $f=0$.

A consequence of Lemma 5.8 is the self-adjointness of $\mathcal{J}_{\alpha}$. 
Lemma 5.9. Let $\alpha>0$ be sufficiently large. Then the operator $\mathcal{J}_{\alpha}$ is self-adjoint.

Proof. By the self-adjointness of $\mathcal{L}_{\alpha,-}^{\frac{1}{2}}, \mathcal{L}_{\alpha,+}$ and Lemma 5.6, it follows that $\mathcal{J}_{\alpha}$ is symmetric. In other words, $\mathcal{J}_{\alpha} \subset \mathcal{J}_{\alpha}^{*}$ and, since $\mathcal{J}_{\alpha}^{*}$ is closed, $\mathcal{J}_{\alpha}$ is closable and its closure $\overline{\mathcal{J}_{\alpha}}$ is symmetric, too. Now consider the operators $\pm i+\mathcal{J}_{\alpha}=\mu_{ \pm}^{2}+\mathcal{J}_{\alpha}$, where $\mu_{ \pm}:=\frac{1}{\sqrt{2}} \pm$ $\frac{i}{\sqrt{2}}$. By Lemma 4.8, $\mu_{ \pm} \in \rho\left(\mathcal{L}_{\alpha}\right)$ and thus, Lemma 5.8 implies that $\pm i+\mathcal{J}_{\alpha}$ is surjective. Consequently, $\pm i+\overline{\mathcal{J}_{\alpha}}$ is surjective and therefore, $\overline{\mathcal{J}_{\alpha}}$ is self-adjoint (see e.g. [43], p. 271, Theorem 3.16). Let $g \in \mathcal{D}\left(\overline{\mathcal{J}_{\alpha}}\right)$ be arbitrary. By the surjectivity of $i+\mathcal{J}_{\alpha}$, there exists an $f \in \mathcal{D}\left(\mathcal{J}_{\alpha}\right)$ such that $\left(i+\mathcal{J}_{\alpha}\right) f=\left(i+\overline{\mathcal{J}_{\alpha}}\right) g$ and $\mathcal{J}_{\alpha} \subset \overline{\mathcal{J}_{\alpha}}$ implies that $\left(i+\overline{\mathcal{J}_{\alpha}}\right)(f-g)=0$. Since $\sigma_{p}\left(\overline{\mathcal{J}_{\alpha}}\right) \subset \mathbb{R}$, we must have $f-g=0$ and therefore, $g \in \mathcal{D}\left(\mathcal{J}_{\alpha}\right)$. Thus, we have proved that $\mathcal{D}\left(\overline{\mathcal{J}_{\alpha}}\right) \subset \mathcal{D}\left(\mathcal{J}_{\alpha}\right)$ and this shows that $\mathcal{J}_{\alpha}=\overline{\mathcal{J}_{\alpha}}$.

We need one last technical result.

Lemma 5.10. Let $\alpha>0$ be sufficiently large and define $\mathcal{B}_{\alpha}: \mathcal{D}\left(\mathcal{B}_{\alpha}\right) \subset\left\langle Q_{\mathbb{R}^{d}}+\rho_{\alpha}\right\rangle^{\perp} \rightarrow$ $\left\langle Q_{\mathbb{R}^{d}}+\rho_{\alpha}\right\rangle^{\perp}$ by $\mathcal{D}\left(\mathcal{B}_{\alpha}\right):=\mathcal{D}\left(\mathcal{L}_{\alpha,-}^{\frac{1}{2}}\right) \cap\left\langle Q_{\mathbb{R}^{d}}+\rho_{\alpha}\right\rangle^{\perp}$ and $\mathcal{B}_{\alpha} f:=\mathcal{L}_{\alpha,-}^{\frac{1}{2}} f$. Then the (maximally defined) operator $\mathcal{L}_{\alpha,+} \mathcal{B}_{\alpha}$ is densely defined, closed, and $\mathcal{D}\left(\mathcal{J}_{\alpha}\right)$ is a core for $\mathcal{L}_{\alpha,+} \mathcal{B}_{\alpha}$.

Proof. Recall from the proof of Lemma 5.6 that $\mathcal{B}_{\alpha}$ is closed and bounded invertible. As a consequence, Lemma 5.5 shows that $\mathcal{L}_{\alpha,+} \mathcal{B}_{\alpha}$ is densely defined. Let $\left(f_{n}\right)_{n \in \mathbb{N}} \subset \mathcal{D}\left(\mathcal{L}_{\alpha,+} \mathcal{B}_{\alpha}\right) \subset$ $\mathcal{D}\left(\mathcal{B}_{\alpha}\right)$ with $f_{n} \rightarrow f$ and $\mathcal{L}_{\alpha,+} \mathcal{B}_{\alpha} f_{n} \rightarrow h$ as $n \rightarrow \infty$. Then we have

$$
\left\|\mathcal{B}_{\alpha} f_{n}-\mathcal{L}_{\alpha,+}^{-1} h\right\|_{L^{2}\left(\mathbb{R}^{d}\right)}=\left\|\mathcal{L}_{\alpha,+}^{-1}\left(\mathcal{L}_{\alpha,+} \mathcal{B}_{\alpha} f_{n}-h\right)\right\|_{L^{2}\left(\mathbb{R}^{d}\right)} \lesssim\left\|\mathcal{L}_{\alpha,+} \mathcal{B}_{\alpha} f_{n}-h\right\|_{L^{2}\left(\mathbb{R}^{d}\right)} \rightarrow 0
$$

as $n \rightarrow \infty$ and the closedness of $\mathcal{B}_{\alpha}$ implies that $f \in \mathcal{D}\left(\mathcal{B}_{\alpha}\right)$ and $\mathcal{B}_{\alpha} f=\mathcal{L}_{\alpha,+}^{-1} h \in \mathcal{D}\left(\mathcal{L}_{\alpha,+}\right)$. Consequently, $f \in \mathcal{D}\left(\mathcal{L}_{\alpha,+} \mathcal{B}_{\alpha}\right)$ and $\mathcal{L}_{\alpha,+} \mathcal{B}_{\alpha} f=\mathcal{L}_{\alpha,+} \mathcal{L}_{\alpha,+}^{-1} h=h$. This proves the closedness of $\mathcal{L}_{\alpha,+} \mathcal{B}_{\alpha}$

Next, we claim that $\mathcal{L}_{\alpha,+} \mathcal{B}_{\alpha}$ has closed range. Indeed, let $\left(h_{n}\right)_{n \in \mathbb{N}} \subset \operatorname{rg}\left(\mathcal{L}_{\alpha,+} \mathcal{B}_{\alpha}\right)$ with $h_{n} \rightarrow$ $h$ as $n \rightarrow \infty$. Then there exists a sequence $\left(f_{n}\right)_{n \in \mathbb{N}} \subset \mathcal{D}\left(\mathcal{L}_{\alpha,+} \mathcal{B}_{\alpha}\right)$ such that $\mathcal{L}_{\alpha,+} \mathcal{B}_{\alpha} f_{n}=h_{n}$. In other words, $f_{n}=\mathcal{B}_{\alpha}^{-1} \mathcal{L}_{\alpha,+}^{-1} h_{n}$ and thus, $f_{n} \rightarrow f$ as $n \rightarrow \infty$ for some $f \in\left\langle Q_{\mathbb{R}^{d}}+\rho_{\alpha}\right\rangle^{\perp}$. By the closedness of $\mathcal{L}_{\alpha,+} \mathcal{B}_{\alpha}$, we infer that $f \in \mathcal{D}\left(\mathcal{L}_{\alpha,+} \mathcal{B}_{\alpha}\right)$ and $\mathcal{L}_{\alpha,+} \mathcal{B}_{\alpha} f=h$, which shows that $h \in \operatorname{rg}\left(\mathcal{L}_{\alpha,+} \mathcal{B}_{\alpha}\right)$.

Now we define an auxiliary operator $\mathcal{A}_{\alpha}: \mathcal{D}\left(\mathcal{A}_{\alpha}\right) \subset\left\langle Q_{\mathbb{R}^{d}}+\rho_{\alpha}\right\rangle^{\perp} \rightarrow \operatorname{rg}\left(\mathcal{L}_{\alpha,+} \mathcal{B}_{\alpha}\right)$ by $\mathcal{D}\left(\mathcal{A}_{\alpha}\right):=\mathcal{D}\left(\mathcal{L}_{\alpha,+} \mathcal{B}_{\alpha}\right)$ and $\mathcal{A}_{\alpha} f:=\mathcal{L}_{\alpha,+} \mathcal{B}_{\alpha} f$. By the above, $\mathcal{A}_{\alpha}$ is densely defined, closed, and bijective. Thus, the closed graph theorem shows that $\mathcal{A}_{\alpha}$ is bounded invertible. By definition, $\mathcal{D}\left(\mathcal{J}_{\alpha}\right)=\mathcal{D}\left(\mathcal{L}_{\alpha,-}^{\frac{1}{2}} \mathcal{A}_{\alpha}\right)$. Lemma 5.5 implies that $\mathcal{D}\left(\mathcal{J}_{\alpha}\right)$ is a core for $\mathcal{A}_{\alpha}$ and hence for $\mathcal{L}_{\alpha,+} \mathcal{B}_{\alpha}$.

5.3. Spectral stability in the critical case. Now we can establish a stability criterion also in the critical case $p=1+\frac{4}{d}$.

Lemma 5.11. If $p=1+\frac{4}{d}$ then there exists an $\alpha_{0}>0$ such that for all $\alpha \geq \alpha_{0}$ the following holds.

- If $\left(\mathcal{L}_{\alpha,+}^{-1}\left(Q_{\mathbb{R}^{d}}+\rho_{\alpha}\right) \mid Q_{\mathbb{R}^{d}}+\rho_{\alpha}\right)_{L^{2}\left(\mathbb{R}^{d}\right)}>0$ then $\mathcal{L}_{\alpha}$ has precisely one positive eigenvalue $\lambda_{\alpha}$ and the eigenvalues $\pm \lambda_{\alpha} \in \sigma_{p}\left(\mathcal{L}_{\alpha}\right)$ are simple.

- If $\left(\mathcal{L}_{\alpha,+}^{-1}\left(Q_{\mathbb{R}^{d}}+\rho_{\alpha}\right) \mid Q_{\mathbb{R}^{d}}+\rho_{\alpha}\right)_{L^{2}\left(\mathbb{R}^{d}\right)} \leq 0$ then $\mathcal{L}_{\alpha}$ has no positive eigenvalues. 
Proof. Let $\mathcal{P}$ and $\mathcal{P}_{\alpha}$ be the spectral projections from the proof of Lemma 4.9. By Theorem 4.3 and [43], p. 34, Lemma 4.10, we have $\operatorname{dim} \operatorname{rg} \mathcal{P}_{\alpha}=\operatorname{dim} \operatorname{rg} \mathcal{P}=4$ and thus, by Lemma 4.8, there can be at most one positive eigenvalue $\lambda_{\alpha}>0$ and if so, the eigenvalues $\pm \lambda_{\alpha} \in \sigma_{p}\left(\mathcal{L}_{\alpha}\right)$ will be simple since $0 \in \sigma_{p}\left(\mathcal{L}_{\alpha}\right)$.

Now assume that $\left(\mathcal{L}_{\alpha,+}^{-1}\left(Q_{\mathbb{R}^{d}}+\rho_{\alpha}\right) \mid Q_{\mathbb{R}^{d}}+\rho_{\alpha}\right)_{L^{2}\left(\mathbb{R}^{d}\right)}>0$. Then, by Proposition 5.3, we can find an $f_{\alpha} \in\left\langle Q_{\mathbb{R}^{d}}+\rho_{\alpha}\right\rangle^{\perp} \cap H_{\text {rad }}^{2}\left(\mathbb{R}^{d}\right)$ such that $\left(\mathcal{L}_{\alpha,+} f_{\alpha} \mid f_{\alpha}\right)_{L^{2}\left(\mathbb{R}^{d}\right)}<0$. From the self-adjointness of $\mathcal{L}_{\alpha,-}$, we have

$$
\operatorname{rg} \mathcal{L}_{\alpha,-}^{\frac{1}{2}}=\left(\operatorname{ker} \mathcal{L}_{\alpha,-}^{\frac{1}{2}}\right)^{\perp}=\operatorname{ker}\left(\mathcal{L}_{\alpha,-}\right)^{\perp}=\left\langle Q_{\mathbb{R}^{d}}+\rho_{\alpha}\right\rangle^{\perp}
$$

Thus, since $f_{\alpha} \perp Q_{\mathbb{R}^{d}}+\rho_{\alpha}$, there exists a $\widetilde{g}_{\alpha} \in \mathcal{D}\left(\mathcal{L}_{\alpha,-}^{\frac{1}{2}}\right)$ such that $\mathcal{L}_{\alpha,-}^{\frac{1}{2}} \widetilde{g}_{\alpha}=f_{\alpha}$. Set $g_{\alpha}:=\mathcal{P}_{\alpha}^{\perp} \widetilde{g}_{\alpha}$, where $\mathcal{P}_{\alpha}^{\perp}$ is the orthogonal projection onto $\left\langle Q_{\mathbb{R}^{d}}+\rho_{\alpha}\right\rangle^{\perp}$. Then we have $g_{\alpha} \in \mathcal{D}\left(\mathcal{B}_{\alpha}\right)$ and $\mathcal{B}_{\alpha} g_{\alpha}=\mathcal{L}_{\alpha,-}^{\frac{1}{2}} \mathcal{P}_{\alpha}^{\perp} \widetilde{g}_{\alpha}=\mathcal{L}_{\alpha,-}^{\frac{1}{2}} \widetilde{g}_{\alpha}=f_{\alpha}$, where $\mathcal{B}_{\alpha}$ is the operator defined in Lemma 5.10. By construction,

$$
\left(\mathcal{L}_{\alpha,+} \mathcal{B}_{\alpha} g_{\alpha} \mid \mathcal{B}_{\alpha} g_{\alpha}\right)_{L^{2}\left(\mathbb{R}^{d}\right)}=\left(\mathcal{L}_{\alpha,+} f_{\alpha} \mid f_{\alpha}\right)_{L^{2}\left(\mathbb{R}^{d}\right)}<0
$$

Since $\mathcal{D}\left(\mathcal{J}_{\alpha}\right)$ is a core for $\mathcal{L}_{\alpha,+} \mathcal{B}_{\alpha}$ (Lemmat5.10), we can find for any given $\epsilon>0$ an $\tilde{f}_{\alpha} \in \mathcal{D}\left(\mathcal{J}_{\alpha}\right)$ such that $\left\|\mathcal{L}_{\alpha,+} \mathcal{B}_{\alpha} \widetilde{f}_{\alpha}-\mathcal{L}_{\alpha,+} \mathcal{B}_{\alpha} g_{\alpha}\right\|_{L^{2}\left(\mathbb{R}^{d}\right)}<\epsilon$ and

$$
\left\|\mathcal{B}_{\alpha} \widetilde{f}_{\alpha}-\mathcal{B}_{\alpha} g_{\alpha}\right\|_{L^{2}\left(\mathbb{R}^{d}\right)}=\left\|\mathcal{L}_{\alpha,+}^{-1}\left[\mathcal{L}_{\alpha,+} \mathcal{B}_{\alpha} \widetilde{f}_{\alpha}-\mathcal{L}_{\alpha,+} \mathcal{B}_{\alpha} g_{\alpha}\right]\right\|_{L^{2}\left(\mathbb{R}^{d}\right)} \lesssim \epsilon .
$$

Consequently, by choosing $\epsilon>0$ sufficiently small, we find

$$
\begin{aligned}
0 & >\left(\mathcal{L}_{\alpha,+} \mathcal{B}_{\alpha} \tilde{f}_{\alpha} \mid \mathcal{B}_{\alpha} \tilde{f}_{\alpha}\right)_{L^{2}\left(\mathbb{R}^{d}\right)}=\left(\mathcal{L}_{\alpha,+} \mathcal{L}_{\alpha,-}^{\frac{1}{2}} \widetilde{f}_{\alpha} \mid \mathcal{L}_{\alpha,-}^{\frac{1}{2}} \tilde{f}_{\alpha}\right)_{L^{2}\left(\mathbb{R}^{d}\right)}=\left(\mathcal{L}_{\alpha,-}^{\frac{1}{2}} \mathcal{L}_{\alpha,+} \mathcal{L}_{\alpha,-}^{\frac{1}{2}} \tilde{f}_{\alpha} \mid \tilde{f}_{\alpha}\right)_{L^{2}\left(\mathbb{R}^{d}\right)} \\
& =\left(\mathcal{J}_{\alpha} \tilde{f}_{\alpha} \mid \tilde{f}_{\alpha}\right)_{L^{2}\left(\mathbb{R}^{d}\right)} .
\end{aligned}
$$

Lemma 5.9 therefore implies that $\mathcal{J}_{\alpha}$ has negative spectrum, i.e., there exists a $\lambda_{\alpha}>0$ such that $-\lambda_{\alpha}^{2} \in \sigma\left(\mathcal{J}_{\alpha}\right)$. If $-\lambda_{\alpha}^{2}-\mathcal{J}_{\alpha}=-\left(\lambda_{\alpha}^{2}+\mathcal{J}_{\alpha}\right)$ is not surjective, then, by Lemma 5.8, $\lambda_{\alpha} \in \sigma\left(\mathcal{L}_{\alpha}\right)$ and by Lemma 4.8, $\lambda_{\alpha} \in \sigma_{p}\left(\mathcal{L}_{\alpha}\right)$. If $-\left(\lambda_{\alpha}^{2}+\mathcal{J}_{\alpha}\right)$ is not injective, Lemma 5.8 implies that $\lambda_{\alpha} \in \sigma_{p}\left(\mathcal{L}_{\alpha}\right)$.

If, on the other hand, $\left(\mathcal{L}_{\alpha,+}^{-1}\left(Q_{\mathbb{R}^{d}}+\rho_{\alpha}\right) \mid Q_{\mathbb{R}^{d}}+\rho_{\alpha}\right)_{L^{2}\left(\mathbb{R}^{d}\right)} \leq 0$, we obtain

$$
\left(\mathcal{J}_{\alpha} f \mid f\right)_{L^{2}\left(\mathbb{R}^{d}\right)}=\left(\mathcal{L}_{\alpha,+} \mathcal{L}_{\alpha,-}^{\frac{1}{2}} f \mid \mathcal{L}_{\alpha,-}^{\frac{1}{2}} f\right)_{L^{2}\left(\mathbb{R}^{d}\right)} \geq 0
$$

for all $f \in \mathcal{D}\left(\mathcal{J}_{\alpha}\right)$, by Proposition 5.3. Thus, Lemma 5.9 implies that $\sigma\left(\mathcal{J}_{\alpha}\right) \subset[0, \infty)$ and from Lemma 5.8 we infer that $\lambda-\mathcal{L}_{\alpha}$ is injective for any $\lambda>0$. Consequently, Lemma 4.8 shows that $\sigma\left(\mathcal{L}_{\alpha}\right) \cap \mathbb{R}=\{0\}$.

Proof of Theorems 1.5, 1.6, and 1.7. Consider the map $\mathcal{V}_{\alpha}: L_{\mathrm{rad}}^{2}\left(\mathbb{M}^{d}\right) \rightarrow L_{\mathrm{rad}}^{2}\left(\mathbb{R}^{d}\right)$ given by

$$
\mathcal{V}_{\alpha} f(x):=\alpha^{-\frac{d}{2}}\left(\frac{A\left(\alpha^{-1}|x|\right)}{\alpha^{-1}|x|}\right)^{\frac{d-1}{2}} f\left(\alpha^{-1}|x|, y\right),
$$

with inverse

$$
\mathcal{V}_{\alpha}^{-1} f(r, y)=\alpha^{\frac{d}{2}}\left(\frac{r}{A(r)}\right)^{\frac{d-1}{2}} f\left(\alpha r e_{1}\right) .
$$


We have

$$
\begin{aligned}
\left\|\mathcal{V}_{\alpha} f\right\|_{L^{2}\left(\mathbb{R}^{d}\right)}^{2} & =\alpha^{-d}\left|\mathbb{S}^{d-1}\right| \int_{0}^{\infty}\left(\frac{A\left(\alpha^{-1} r\right)}{\alpha^{-1} r}\right)^{d-1}\left|f\left(\alpha^{-1} r, y\right)\right|^{2} r^{d-1} d r \\
& =\left|\mathbb{S}^{d-1}\right| \int_{0}^{\infty}|f(r, y)|^{2} A(r)^{d-1} d r \\
& =\|f\|_{L^{2}\left(\mathbb{M}^{d}\right)}^{2},
\end{aligned}
$$

and thus, $\mathcal{V}_{\alpha}$ is unitary for any $\alpha>0$. Furthermore, recall that

$$
Q_{\mathbb{M}^{d}, \alpha}(r, y)=\alpha^{\frac{2}{p-1}}\left(\frac{r}{A(r)}\right)^{\frac{d-1}{2}}\left[Q_{\mathbb{R}^{d}}\left(\alpha r e_{1}\right)+\rho_{\alpha}\left(\alpha r e_{1}\right)\right]
$$

and thus, for any radial $f \in C_{c}^{\infty}\left(\mathbb{M}^{d}\right)$, we have

$$
\widetilde{\mathcal{L}}_{\mathbb{M}^{d}, \alpha, \pm} f=\alpha^{2} \mathcal{V}_{\alpha}^{-1} \mathcal{L}_{\alpha, \pm} \mathcal{V}_{\alpha} f
$$

Consequently, the closure $\mathcal{L}_{\mathbb{M}^{d}, \alpha}$ of $\widetilde{\mathcal{L}}_{\mathbb{M}^{d}, \alpha}$ is given by

$$
\mathcal{L}_{\mathbb{M}^{d}, \alpha}=\alpha^{2}\left(\begin{array}{cc}
0 & \mathcal{V}_{\alpha}^{-1} \mathcal{L}_{\alpha,-} \mathcal{V}_{\alpha} \\
-\mathcal{V}_{\alpha}^{-1} \mathcal{L}_{\alpha,+} \mathcal{V}_{\alpha} & 0
\end{array}\right)
$$

and $\mathcal{L}_{\mathbb{M}^{d}, \alpha}$ is unitarily equivalent to $\alpha^{2} \mathcal{L}_{\alpha}$. This implies the claimed statements.

\section{Stability And CuRvature}

From [4], we know that in negative curvature there is blow-up instability for sufficiently high energy. In this last section we give numerical evidence of how this instability manifests in the bifurcation theory from the Euclidean situation. The soliton may become linearly unstable in the curved geometry if the curvature is strictly negative everywhere or otherwise. More precisely, we consider the model case of a warping function $A(r)=r+c_{1} r^{3}+c_{2} r^{5}$, in the critical case $d=2, p=3$. The sectional curvatures of the manifold $\mathbb{M}^{2}$ are given by

$$
-\frac{A^{\prime \prime}(r)}{A(r)}=-\frac{6 c_{1}+20 c_{2} r^{2}}{1+c_{1} r^{2}+c_{2} r^{4}}, \quad \frac{1-A^{\prime}(r)^{2}}{A(r)^{2}}=-\frac{\left(3 c_{1}+5 c_{2} r^{2}\right)\left(2+3 c_{1} r^{2}+5 c_{2} r^{4}\right)}{\left(1+c_{1} r^{2}+c_{2} r^{4}\right)^{2}} .
$$

6.1. A formal expansion. As before, we write $\widetilde{R}_{\alpha}=Q_{\mathbb{R}^{2}}+\rho_{\alpha}$ with $\rho_{\alpha}$ from Proposition 3.7. By Lemma 3.9, $\widetilde{R}_{\alpha} \in C^{2}\left(\mathbb{R}^{2}\right)$ and

$$
\Delta_{\mathbb{R}^{2}} \widetilde{R}_{\alpha}(x)-\widetilde{R}_{\alpha}(x)-\alpha^{-2} V_{2}\left(\alpha^{-1} x\right) \widetilde{R}_{\alpha}(x)+\varphi_{2,3}\left(\alpha^{-1} x\right) \widetilde{R}_{\alpha}(x)^{3}=0
$$

for all $x \in \mathbb{R}^{2}$. When written out explicitly for our model case, this reads

$$
\begin{aligned}
& \left(1+c_{1} \alpha^{-2} r^{2}+c_{2} \alpha^{-4} r^{4}\right)^{2}\left(\Delta_{\mathbb{R}^{2}}-1\right) \widetilde{R}_{\alpha} \\
& \quad-\alpha^{-2}\left[2 c_{1}+\left(c_{1}^{2}+8 c_{2}\right) \alpha^{-2} r^{2}+6 c_{1} c_{2} \alpha^{-4} r^{4}+4 c_{2}^{2} \alpha^{-6} r^{6}\right] \widetilde{R}_{\alpha} \\
& \quad+\left(1+c_{1} \alpha^{-2} r^{2}+c_{2} \alpha^{-4} r^{4}\right) \widetilde{R}_{\alpha}^{3}=0,
\end{aligned}
$$

where $r(x)=|x|$. Now we assume an asymptotic expansion of the form

$$
\widetilde{R}_{\alpha}(x)=Q_{\mathbb{R}^{2}}(x)+\alpha^{-2} Q_{1}(x)+\alpha^{-4} Q_{2}(x)+\alpha^{-6} Q_{E}(x, \alpha),
$$


where $\left\|Q_{E}(\cdot, \alpha)\right\|_{L^{2}\left(\mathbb{R}^{2}\right)} \lesssim 1$ and $\left\|\partial_{\alpha} Q_{E}(\cdot, \alpha)\right\|_{L^{2}\left(\mathbb{R}^{2}\right)} \lesssim \alpha^{-1}$ for all $\alpha \gg 1$. Then, in view of Theorem [1.3, the soliton profile on $\mathbb{M}^{2}$ is given by

$$
Q_{\mathbb{M}^{2}, \alpha}(r, y)=\alpha\left(\frac{r}{A(r)}\right)^{\frac{1}{2}}\left[Q_{\mathbb{R}^{2}}\left(\alpha r e_{1}\right)+\alpha^{-2} Q_{1}\left(\alpha r e_{1}\right)+\alpha^{-4} Q_{2}\left(\alpha r e_{1}\right)+\alpha^{-6} Q_{E}\left(\alpha r e_{1}, \alpha\right)\right]
$$

and thus,

$$
\begin{aligned}
\left\|Q_{\mathbb{M}^{2}, \alpha}\right\|_{L^{2}\left(\mathbb{M}^{2}\right)}^{2}= & \int_{0}^{\infty} \int_{\mathbb{R}} Q_{\mathbb{M}^{2}, \alpha}(r, y)^{2} A(r) \frac{2}{y^{2}+1} d y d r=2 \pi \int_{0}^{\infty} Q_{\mathbb{M}^{2}, \alpha}(r, y)^{2} A(r) d r \\
= & 2 \pi \int_{0}^{\infty}\left[Q_{\mathbb{R}^{2}}\left(r e_{1}\right)+\alpha^{-2} Q_{1}\left(r e_{1}\right)+\alpha^{-4} Q_{2}\left(r e_{1}\right)+\alpha^{-6} Q_{E}\left(r e_{1}, \alpha\right)\right]^{2} r d r \\
= & \left\|Q_{\mathbb{R}^{2}}\right\|_{L^{2}\left(\mathbb{R}^{2}\right)}^{2}+2 \alpha^{-2}\left(Q_{\mathbb{R}^{2}} \mid Q_{1}\right)_{L^{2}\left(\mathbb{R}^{2}\right)}+\alpha^{-4}\left[\left\|Q_{1}\right\|_{L^{2}\left(\mathbb{R}^{2}\right)}^{2}+2\left(Q_{\mathbb{R}^{2}} \mid Q_{2}\right)_{L^{2}\left(\mathbb{R}^{2}\right)}\right] \\
& +O\left(\alpha^{-6}\right) .
\end{aligned}
$$

In order to compute the profiles $Q_{1}$ and $Q_{2}$, we plug the ansatz (6.2) into Eq. (6.1) and solve order by order in $\alpha$. This yields

$$
\begin{aligned}
\alpha^{0} & : \Delta_{\mathbb{R}^{2}} Q_{\mathbb{R}^{2}}-Q_{\mathbb{R}^{2}}+Q_{\mathbb{R}^{2}}^{3}=0, \\
\alpha^{-2} & : \mathcal{L}_{+} Q_{1}=-c_{1}\left(2 Q_{\mathbb{R}^{2}}+r^{2} Q_{\mathbb{R}^{2}}^{3}\right), \\
\alpha^{-4} & : \mathcal{L}_{+} Q_{2}=-2 c_{1} Q_{1}+\left(3 c_{1}^{2}-8 c_{2}\right) r^{2} Q_{\mathbb{R}^{2}}-3 c_{1} r^{2} Q_{\mathbb{R}^{2}}^{2} Q_{1}+3 Q_{\mathbb{R}^{2}} Q_{1}^{2}+\left(c_{1}^{2}-c_{2}\right) r^{4} Q_{\mathbb{R}^{2}}^{3},
\end{aligned}
$$

with $\mathcal{L}_{+}=-\Delta_{\mathbb{R}^{2}}+1-3 Q_{\mathbb{R}^{2}}^{2}$. By definition, $Q_{\mathbb{R}^{2}, \alpha}(x)=\alpha Q_{\mathbb{R}^{2}}(\alpha x)$ satisfies

$$
\Delta_{\mathbb{R}^{2}} Q_{\mathbb{R}^{2}, \alpha}-\alpha^{2} Q_{\mathbb{R}^{2}, \alpha}+Q_{\mathbb{R}^{2}, \alpha}^{3}=0
$$

By differentiating this equation with respect to $\alpha$, we see that

$$
S_{0}(x):=\left.\partial_{\alpha} Q_{\mathbb{R}^{2}, \alpha}(x)\right|_{\alpha=1}=x^{j} \partial_{j} Q_{\mathbb{R}^{2}}(x)+Q_{\mathbb{R}^{2}}(x)
$$

satisfies

Consequently,

$$
\mathcal{L}_{+} S_{0}=-\Delta_{\mathbb{R}^{2}} S_{0}+S_{0}-3 Q_{\mathbb{R}^{2}}^{2} S_{0}=-2 Q_{\mathbb{R}^{2}}
$$

$$
\begin{aligned}
\left(Q_{\mathbb{R}^{2}} \mid Q_{1}\right)_{L^{2}\left(\mathbb{R}^{2}\right)} & =-\frac{1}{2}\left(\mathcal{L}_{+} S_{0} \mid Q_{1}\right)_{L^{2}\left(\mathbb{R}^{2}\right)}=-\frac{1}{2}\left(S_{0} \mid \mathcal{L}_{+} Q_{1}\right)_{L^{2}\left(\mathbb{R}^{2}\right)} \\
& =c_{1}\left(S_{0} \mid Q_{\mathbb{R}^{2}}\right)_{L^{2}\left(\mathbb{R}^{2}\right)}+\frac{1}{2} c_{1}\left(S_{0} \mid r^{2} Q_{\mathbb{R}^{2}}^{3}\right)_{L^{2}\left(\mathbb{R}^{2}\right)} \\
& =0
\end{aligned}
$$

since

and

$$
0=\partial_{\alpha}\left\|Q_{\mathbb{R}^{2}}\right\|_{L^{2}\left(\mathbb{R}^{2}\right)}^{2}=\partial_{\alpha}\left\|Q_{\mathbb{R}^{2}, \alpha}\right\|_{L^{2}\left(\mathbb{R}^{2}\right)}^{2}=2\left(\partial_{\alpha} Q_{\mathbb{R}^{2}, \alpha} \mid Q_{\mathbb{R}^{2}, \alpha}\right)_{L^{2}\left(\mathbb{R}^{2}\right)}
$$

$$
0=\partial_{\alpha}\left\|r Q_{\mathbb{R}^{2}}^{2}\right\|_{L^{2}\left(\mathbb{R}^{2}\right)}^{2}=\partial_{\alpha}\left\|r Q_{\mathbb{R}^{2}, \alpha}^{2}\right\|_{L^{2}\left(\mathbb{R}^{2}\right)}^{2}=4\left(\partial_{\alpha} Q_{\mathbb{R}^{2}, \alpha} \mid r^{2} Q_{\mathbb{R}^{2}, \alpha}^{3}\right)_{L^{2}\left(\mathbb{R}^{2}\right)}
$$

which, when evaluated at $\alpha=1$, reads

$$
0=\left(S_{0} \mid Q_{\mathbb{R}^{2}}\right)_{L^{2}\left(\mathbb{R}^{2}\right)}=\left(S_{0} \mid r^{2} Q_{\mathbb{R}^{2}}^{3}\right)_{L^{2}\left(\mathbb{R}^{2}\right)}
$$

This implies that

$$
\left\|Q_{\mathbb{M}^{2}, \alpha}\right\|_{L^{2}\left(\mathbb{M}^{2}\right)}^{2}=\left\|Q_{\mathbb{R}^{2}}\right\|_{L^{2}\left(\mathbb{R}^{2}\right)}^{2}+\alpha^{-4}\left[\left\|Q_{1}\right\|_{L^{2}\left(\mathbb{R}^{2}\right)}^{2}+2\left(Q_{\mathbb{R}^{2}} \mid Q_{2}\right)_{L^{2}\left(\mathbb{R}^{2}\right)}\right]+O\left(\alpha^{-6}\right) .
$$


The sign of $\partial_{\alpha}\left\|Q_{\mathbb{M}^{2}, \alpha}\right\|_{L^{2}\left(\mathbb{R}^{2}\right)}^{2}$ is to leading order determined by the sign of

$$
\kappa:=\left\|Q_{1}\right\|_{L^{2}\left(\mathbb{R}^{2}\right)}^{2}+2\left(Q_{\mathbb{R}^{2}} \mid Q_{2}\right)_{L^{2}\left(\mathbb{R}^{2}\right)} .
$$

More precisely, we have

$$
\partial_{\alpha}\left\|Q_{\mathbb{M}^{2}, \alpha}\right\|_{L^{2}\left(\mathbb{M}^{2}\right)}^{2}=-4 \alpha^{-5} \kappa+O\left(\alpha^{-7}\right)
$$

and the soliton is linearly unstable for sufficiently large $\alpha$ if $\kappa>0$, see Theorem 1.7 and Remark 1.8.

6.2. Stability. By using the defining equation for $Q_{2}$, we find the expression

$$
\begin{aligned}
\kappa= & \left\|Q_{1}\right\|_{L^{2}\left(\mathbb{R}^{2}\right)}^{2}-\left(\mathcal{L}_{+} S_{0} \mid Q_{2}\right)_{L^{2}\left(\mathbb{R}^{2}\right)}=\left\|Q_{1}\right\|_{L^{2}\left(\mathbb{R}^{2}\right)}^{2}-\left(S_{0} \mid \mathcal{L}_{+} Q_{2}\right)_{L^{2}\left(\mathbb{R}^{2}\right)} \\
= & \left\|Q_{1}\right\|_{L^{2}\left(\mathbb{R}^{2}\right)}^{2} \\
& +\left(S_{0} \mid 2 c_{1} Q_{1}-\left(3 c_{1}^{2}-8 c_{2}\right) r^{2} Q_{\mathbb{R}^{2}}+3 c_{1} r^{2} Q_{\mathbb{R}^{2}}^{2} Q_{1}-3 Q_{\mathbb{R}^{2}} Q_{1}^{2}-\left(c_{1}^{2}-c_{2}\right) r^{4} Q_{\mathbb{R}^{2}}^{3}\right)_{L^{2}\left(\mathbb{R}^{2}\right)} .
\end{aligned}
$$

It is convenient to introduce the function $\widehat{Q}_{1}$, defined as the unique solution $\left(\right.$ in $H_{\text {rad }}^{2}\left(\mathbb{R}^{2}\right)$ ) of the equation

$$
\mathcal{L}_{+} \widehat{Q}_{1}=-2 Q_{\mathbb{R}^{2}}-r^{2} Q_{\mathbb{R}^{2}}^{3} .
$$

Then we have $Q_{1}=c_{1} \widehat{Q}_{1}$ and we arrive at $\kappa=c_{1}^{2} b_{1}+c_{2} b_{2}$ with

$$
\begin{aligned}
& b_{1}=\left\|\widehat{Q}_{1}\right\|_{L^{2}\left(\mathbb{R}^{2}\right)}^{2}+\left(S_{0} \mid 2 \widehat{Q}_{1}-3 r^{2} Q_{\mathbb{R}^{2}}+3 r^{2} Q_{\mathbb{R}^{2}}^{2} \widehat{Q}_{1}-3 Q_{\mathbb{R}^{2}} \widehat{Q}_{1}^{2}-r^{4} Q_{\mathbb{R}^{2}}^{3}\right)_{L^{2}\left(\mathbb{R}^{2}\right)}, \\
& b_{2}=\left(S_{0} \mid 8 r^{2} Q_{\mathbb{R}^{2}}+r^{4} Q_{\mathbb{R}^{2}}^{3}\right)_{L^{2}\left(\mathbb{R}^{2}\right)} .
\end{aligned}
$$

Consequently, the issue is to determine the signs of $b_{1}$ and $b_{2}$ (which depend only on the Euclidean profile $Q_{\mathbb{R}^{2}}$ ). An integration by parts yields

$$
b_{2}=-8\left(Q_{\mathbb{R}^{2}} \mid r^{2} Q_{\mathbb{R}^{2}}\right)_{L^{2}\left(\mathbb{R}^{2}\right)}-\frac{1}{2}\left(Q_{\mathbb{R}^{2}} \mid r^{4} Q_{\mathbb{R}^{2}}^{3}\right)_{L^{2}\left(\mathbb{R}^{2}\right)}<0
$$

and numerical evaluation shows, somewhat surprisingly, that $b_{1} \geq 14 \pi$, see Appendix C. This means that the simple choice $c_{1}=1$ and $c_{2}=0$ provides a negatively curved metric that makes the soliton linearly unstable. In addition, we see that there are values of $c_{1} \ll c_{2}$ such that the mass condition for stability is possibly true. Of course, to establish orbital stability, further analysis is required on such a manifold, for which the metric expansion is far from standard examples.

\section{Appendix A. BACKGRound MATERIAL}

For the convenience of the reader and to fix notation, we compile some background material on radial distributions and distributional solutions of Poisson's equation.

A.1. Radial distributions. As usual, for $U \subset \mathbb{R}^{d}$ open, we denote by $\mathcal{D}(U)=C_{c}^{\infty}(U)$ the set of test functions. For $\left(\varphi_{n}\right)_{n \in \mathbb{N}} \subset \mathcal{D}(U)$ and $\varphi \in \mathcal{D}(U)$, we say that $\lim _{n \rightarrow \infty} \varphi_{n}=\varphi$ in $\mathcal{D}(U)$ if there exists a compact $K \subset U$ such that $\operatorname{supp} \varphi_{n} \subset K$ for all $n \in \mathbb{N}$ and for any $k \in \mathbb{N}_{0},\left\|\varphi_{n}-\varphi\right\|_{W^{k, \infty}(U)} \rightarrow 0$ as $n \rightarrow \infty$. Here,

$$
\|\varphi\|_{W^{k, \infty}(U)}=\sum_{\substack{|\beta| \leq k \\ 35}}\left\|\partial^{\beta} \varphi\right\|_{L^{\infty}(U)}
$$


with the usual multi-index notation. This notion of convergence defines a topology on $\mathcal{D}(U)$ and the space $\mathcal{D}^{\prime}(U)$ of continuous linear functionals on $\mathcal{D}(U)$ is called the space of distributions.

In order to define radial distributions, we start with a test function $f \in \mathcal{D}\left(\mathbb{R}^{d}\right)$ and define its spherical mean $M f$ by

$$
M f(x):=\frac{1}{\left|\mathbb{S}^{d-1}\right|} \int_{\mathbb{S}^{d-1}} f(|x| \omega) d \sigma(\omega)
$$

where $\sigma$ is the standard surface measure on the sphere $\mathbb{S}^{d-1}$. Clearly, $M f=f$ if and only if $f$ is radial. The most important properties are summarized in the next lemma.

Lemma A.1. We have $\Delta_{\mathbb{R}^{d}} M f=M \Delta_{\mathbb{R}^{d}} f$ for all $f \in C_{c}^{\infty}\left(\mathbb{R}^{d}\right)$. Furthermore, for any $s \geq 0$ we have

$$
\|M f\|_{H^{s}\left(\mathbb{R}^{d}\right)} \lesssim\|f\|_{H^{s}\left(\mathbb{R}^{d}\right)}
$$

for all $f \in C_{c}^{\infty}\left(\mathbb{R}^{d}\right)$. Finally, $M$ extends to a self-adjoint operator on $L^{2}\left(\mathbb{R}^{d}\right)$.

Proof. We use polar coordinates $x=r \omega^{\prime}$ defined by $r=|x|, \omega^{\prime}=\frac{x}{|x|}$ for $x \in \mathbb{R}^{d} \backslash\{0\}$. Since $M f$ is radial, we obtain

$$
\begin{aligned}
\Delta_{\mathbb{R}^{d}} M f(x) & =\left(\partial_{r}^{2}+\frac{d-1}{r} \partial_{r}\right) M f\left(r \omega^{\prime}\right)=\frac{1}{\left|\mathbb{S}^{d-1}\right|}\left(\partial_{r}^{2}+\frac{d-1}{r} \partial_{r}\right) \int_{\mathbb{S}^{d-1}} f(r \omega) d \sigma(\omega) \\
& =\frac{1}{\left|\mathbb{S}^{d-1}\right|} \int_{\mathbb{S}^{d-1}}\left(\partial_{r}^{2}+\frac{d-1}{r} \partial_{r}\right) f(r \omega) d \sigma(\omega) \\
& =\frac{1}{\left|\mathbb{S}^{d-1}\right|} \int_{\mathbb{S}^{d-1}}\left(\partial_{r}^{2}+\frac{d-1}{r} \partial_{r}+\frac{1}{r^{2}} \Delta_{\mathbb{S}^{d-1}, \omega}\right) f(r \omega) d \sigma(\omega) \\
& =\frac{1}{\left|\mathbb{S}^{d-1}\right|} \int_{\mathbb{S}^{d-1}} \Delta_{\mathbb{R}^{d}} f(r \omega) d \sigma(\omega) \\
& =M \Delta_{\mathbb{R}^{d}} f(x)
\end{aligned}
$$

for any $x \in \mathbb{R}^{d} \backslash\{0\}$. Next, by Cauchy-Schwarz,

$$
\begin{aligned}
\|M f\|_{L^{2}\left(\mathbb{R}^{d}\right)}^{2} & =\int_{0}^{\infty} \int_{\mathbb{S}^{d-1}}|M f(r \omega)|^{2} d \sigma(\omega) r^{d-1} d r \\
& \lesssim \int_{0}^{\infty} \int_{\mathbb{S}^{d-1}} \int_{\mathbb{S}^{d-1}}\left|f\left(r \omega^{\prime}\right)\right|^{2} d \sigma\left(\omega^{\prime}\right) d \sigma(\omega) r^{d-1} d r \\
& \lesssim\|f\|_{L^{2}\left(\mathbb{R}^{d}\right)}^{2}
\end{aligned}
$$

Since $M$ commutes with $\Delta_{\mathbb{R}^{d}}$, we can also estimate

$$
\begin{aligned}
\|M f\|_{H^{2 k}\left(\mathbb{R}^{d}\right)} & \simeq\left\|\Delta_{\mathbb{R}^{d}}^{k} M f\right\|_{L^{2}\left(\mathbb{R}^{d}\right)}+\|M f\|_{L^{2}\left(\mathbb{R}^{d}\right)}=\left\|M \Delta_{\mathbb{R}^{d}}^{k} f\right\|_{L^{2}\left(\mathbb{R}^{d}\right)}+\|M f\|_{L^{2}\left(\mathbb{R}^{d}\right)} \\
& \lesssim\left\|\Delta_{\mathbb{R}^{d}}^{k} f\right\|_{L^{2}\left(\mathbb{R}^{d}\right)}+\|f\|_{L^{2}\left(\mathbb{R}^{d}\right)} \\
& \simeq\|f\|_{H^{2 k}\left(\mathbb{R}^{d}\right)}
\end{aligned}
$$

for any $k \in \mathbb{N}_{0}$. By interpolation, we obtain the claimed estimate. Clearly, $M f$ has compact support if $f \in C_{c}^{\infty}\left(\mathbb{R}^{d}\right)$ and the Sobolev embedding theorem shows that in fact $M f \in$ $C_{c}^{\infty}\left(\mathbb{R}^{d}\right)$. In particular, $\Delta_{\mathbb{R}^{d}} M f(x)=M \Delta_{\mathbb{R}^{d}} f(x)$ holds for all $x \in \mathbb{R}^{d}$ by continuity. 
By density, $M$ extends to a bounded operator on $L^{2}\left(\mathbb{R}^{d}\right)$, and we have

$$
\begin{aligned}
(M f \mid g)_{L^{2}\left(\mathbb{R}^{d}\right)} & =\int_{\mathbb{R}^{d}} M f(x) \overline{g(x)} d x=\int_{0}^{\infty} \int_{\mathbb{S}^{d-1}} M f(r \omega) \overline{g(r \omega)} d \sigma(\omega) r^{d-1} d r \\
& =\frac{1}{\left|\mathbb{S}^{d-1}\right|} \int_{0}^{\infty} \int_{\mathbb{S}^{d-1}} \int_{\mathbb{S}^{d-1}} f\left(r \omega^{\prime}\right) d \sigma\left(\omega^{\prime}\right) \overline{g(r \omega)} d \sigma(\omega) r^{d-1} d r \\
& =\frac{1}{\left|\mathbb{S}^{d-1}\right|} \int_{0}^{\infty} \int_{\mathbb{S}^{d-1}} f\left(r \omega^{\prime}\right) \int_{\mathbb{S}^{d-1}} \overline{g(r \omega)} d \sigma(\omega) d \sigma\left(\omega^{\prime}\right) r^{d-1} d r \\
& =\int_{0}^{\infty} f\left(r \omega^{\prime}\right) \overline{M g\left(r \omega^{\prime}\right)} d \sigma\left(\omega^{\prime}\right) r^{d-1} d r \\
& =(f \mid M g)_{L^{2}\left(\mathbb{R}^{d}\right)}
\end{aligned}
$$

for all $f, g \in C_{c}^{\infty}\left(\mathbb{R}^{d}\right)$ by Fubini. Consequently, $M$ extends to a self-adjoint operator on $L^{2}\left(\mathbb{R}^{d}\right)$.

We use the same symbol $M$ to denote the extension of the spherical mean to $L^{2}\left(\mathbb{R}^{d}\right)$. For $s \geq 0$ we define the closed subspace $H_{\text {rad }}^{s}\left(\mathbb{R}^{d}\right) \subset H^{s}\left(\mathbb{R}^{d}\right)$ of radial functions in $H^{s}\left(\mathbb{R}^{d}\right)$ by

$$
H_{\text {rad }}^{s}\left(\mathbb{R}^{d}\right):=\left\{f \in H^{s}\left(\mathbb{R}^{d}\right): M f=f\right\} .
$$

It is now straightforward to further extend $M$ to distributions. Indeed, for $u \in \mathcal{D}^{\prime}\left(\mathbb{R}^{d}\right)$ we define $\widehat{M} u$ by

$$
(\widehat{M} u)(\varphi):=u(M \varphi)
$$

for all $\varphi \in \mathcal{D}\left(\mathbb{R}^{d}\right)$. Obviously, $\widehat{M} u$ is a linear form on $\mathcal{D}\left(\mathbb{R}^{d}\right)$ and, for any $K \subset \mathbb{R}^{d}$ compact, we can find a $k \in \mathbb{N}_{0}$ such that

$$
|(\widehat{M} u)(\varphi)|=|u(M \varphi)| \lesssim\|M \varphi\|_{W^{k, \infty}\left(\mathbb{R}^{d}\right)} \lesssim\|M \varphi\|_{H^{k+d}\left(\mathbb{R}^{d}\right)} \lesssim\|\varphi\|_{H^{k+d}\left(\mathbb{R}^{d}\right)} \lesssim\|\varphi\|_{W^{k+d, \infty}(K)}
$$

for all $\varphi \in C_{c}^{\infty}(K)$ by Sobolev embedding and Lemma A.1. This estimate shows that $\widehat{M} u \in \mathcal{D}^{\prime}\left(\mathbb{R}^{d}\right)$ and by the self-adjointness of $M$ on $L^{2}\left(\mathbb{R}^{d}\right)$, the operator $\widehat{M}: \mathcal{D}^{\prime}\left(\mathbb{R}^{d}\right) \rightarrow \mathcal{D}^{\prime}\left(\mathbb{R}^{d}\right)$ is an extension of $M$ to the space of distributions. Consequently, it is justified to simplify notation by writing $M$ instead of $\widehat{M}$. Accordingly, a distribution $u \in \mathcal{D}^{\prime}\left(\mathbb{R}^{d}\right)$ is said to be radial if $M u=u$. Note that by Lemma A.1, $\Delta_{\mathbb{R}^{d}}$ maps radial distributions to radial distributions.

A.2. Regularity results. We state and prove two regularity results for radial distributional solutions of Poisson's equation. It is convenient to introduce the following notation.

Definition A.2. Let $U \subset \mathbb{R}^{d}$ be open and $f \in L_{\text {loc }}^{1}(U)$. Then we define the distribution $f^{\sharp} \in \mathcal{D}^{\prime}(U)$ by

$$
f^{\sharp}(\varphi):=\int_{U} f(x) \varphi(x) d x
$$

for $\varphi \in \mathcal{D}(U)$.

Lemma A.3. Let $f, g \in C\left(\mathbb{R}^{d} \backslash\{0\}\right) \cap L_{\mathrm{loc}}^{1}\left(\mathbb{R}^{d}\right)$ be radial and suppose $f$ satisfies

$$
\Delta_{\mathbb{R}^{d}} f^{\sharp}=g_{37}^{\sharp} \text { in } \mathcal{D}^{\prime}\left(\mathbb{R}^{d}\right) \text {. }
$$


Then the function $\widehat{f}:(0, \infty) \rightarrow \mathbb{R}$, defined by $\widehat{f}(r):=r^{\frac{d-1}{2}} f\left(r e_{1}\right)$, belongs to $C^{2}(0, \infty)$ and satisfies

$$
\widehat{f}^{\prime \prime}(r)-\frac{(d-1)(d-3)}{4 r^{2}} \widehat{f}(r)=r^{\frac{d-1}{2}} g\left(r e_{1}\right)
$$

for all $r>0$.

Proof. Let

$$
\mathcal{D}_{d} \psi(r):=\psi^{\prime \prime}(r)-\frac{(d-1)(d-3)}{4 r^{2}} \psi(r) .
$$

The operator $\mathcal{D}_{d}$ maps $\mathcal{D}(0, \infty)$ to $\mathcal{D}(0, \infty)$ continuously and is formally self-adjoint on $L^{2}(0, \infty)$. Thus, $\mathcal{D}_{d}$ extends to $\mathcal{D}^{\prime}(0, \infty)$ by setting $\mathcal{D}_{d} v(\psi):=v\left(\mathcal{D}_{d} \psi\right)$ for $v \in \mathcal{D}^{\prime}(0, \infty)$ and $\psi \in \mathcal{D}(0, \infty)$. Furthermore, we have the identity

$$
\Delta_{\mathbb{R}^{d}}\left(|\cdot|^{-\frac{d-1}{2}} \psi(|\cdot|)\right)(x)=|x|^{-\frac{d-1}{2}}\left(\mathcal{D}_{d} \psi\right)(|x|)
$$

for all $x \in \mathbb{R}^{d}$. Now note that $\psi \in C_{c}^{\infty}(0, \infty)$ implies $|\cdot|^{-\frac{d-1}{2}} \psi(|\cdot|) \in C_{c}^{\infty}\left(\mathbb{R}^{d}\right)$ and thus, every distribution $u \in \mathcal{D}^{\prime}\left(\mathbb{R}^{d}\right)$ defines a distribution $\widehat{u} \in \mathcal{D}^{\prime}(0, \infty)$ by setting

$$
\widehat{u}(\psi):=u\left(|\cdot|^{-\frac{d-1}{2}} \psi(|\cdot|)\right)
$$

for $\psi \in \mathcal{D}(0, \infty)$. Then we have

$$
\begin{aligned}
\Delta_{\mathbb{R}^{d}} u\left(|\cdot|^{-\frac{d-1}{2}} \psi(|\cdot|)\right) & =u\left(\Delta_{\mathbb{R}^{d}}\left(|\cdot|^{-\frac{d-1}{2}} \psi(|\cdot|)\right)\right) \\
& =u\left(|\cdot|^{-\frac{d-1}{2}}\left(\mathcal{D}_{d} \psi\right)(|\cdot|)\right)=\widehat{u}\left(\mathcal{D}_{d} \psi\right) \\
& =\mathcal{D}_{d} \widehat{u}(\psi)
\end{aligned}
$$

for all $\psi \in \mathcal{D}(0, \infty)$, and the equation $\Delta_{\mathbb{R}^{d}} f^{\sharp}=g^{\sharp}$ in $\mathcal{D}^{\prime}\left(\mathbb{R}^{d}\right)$ implies that

$$
\mathcal{D}_{d} \widehat{f^{\sharp}}=\widehat{g^{\sharp}} \text { in } \mathcal{D}^{\prime}(0, \infty) \text {. }
$$

Explicitly, we have

$$
\widehat{f^{\sharp}}(\psi)=f^{\sharp}\left(|\cdot|^{-\frac{d-1}{2}} \psi(|\cdot|)\right)=\int_{\mathbb{R}^{d}} f(x)|x|^{-\frac{d-1}{2}} \psi(|x|) d x=\left|\mathbb{S}^{d-1}\right| \int_{0}^{\infty} r^{\frac{d-1}{2}} f\left(r e_{1}\right) \psi(r) d r
$$

and thus, $\widehat{f^{\sharp}}=\hat{f}^{\sharp}$ with $\hat{f}(r):=\left|\mathbb{S}^{d-1}\right| r^{\frac{d-1}{2}} f\left(r e_{1}\right)$. This yields

$$
\mathcal{D}_{d} \hat{f}^{\sharp}=\hat{g}^{\sharp} \text { in } \mathcal{D}^{\prime}(0, \infty),
$$

and by 41, p. 58, Corollary 3.1.6, it follows that $\hat{f} \in C^{2}(0, \infty)$ and $\mathcal{D}_{d} \hat{f}=\hat{g}$ holds in the classical sense.

Lemma A.4. Let $f, g \in C\left(\mathbb{R}^{d}\right)$ be radial and suppose $f$ satisfies

$$
\Delta_{\mathbb{R}^{d}} f^{\sharp}=g^{\sharp} \text { in } \mathcal{D}^{\prime}\left(\mathbb{R}^{d}\right) .
$$

Then $f \in C^{2}\left(\mathbb{R}^{d}\right)$ and $\Delta_{\mathbb{R}^{d}} f(x)=g(x)$ for all $x \in \mathbb{R}^{d}$ in the classical sense. 
Proof. From Lemma A.3 we know that $\widehat{f}(r):=r^{\frac{d-1}{2}} f\left(r e_{1}\right)$ belongs to $C^{2}(0, \infty)$ and satisfies

$$
\widehat{f}^{\prime \prime}(r)-\frac{(d-1)(d-3)}{4 r^{2}} \widehat{f}(r)=r^{\frac{d-1}{2}} g\left(r e_{1}\right)
$$

for all $r>0$. In particular, $f \in C^{2}\left(\mathbb{R}^{d} \backslash\{0\}\right)$. We set $\phi(s):=s^{-\frac{d-1}{4}} \widehat{f}(\sqrt{s})=f\left(\sqrt{s} e_{1}\right)$. Then $\phi \in C^{2}(0, \infty) \cap C([0, \infty))$ and

$$
\phi^{\prime \prime}(s)+\frac{d}{2 s} \phi^{\prime}(s)=\frac{1}{s} h(s)
$$

for all $s>0$ and $h(s):=\frac{1}{4} g\left(\sqrt{s} e_{1}\right)$. Obviously, $h \in C([0, \infty))$. A fundamental system for the homogeneous equation is given by $\left\{1, \psi_{0}\right\}$, where $\psi_{0}(s)=-\frac{2}{d-2} s^{-\frac{d-2}{2}}$ if $d \geq 3$ and $\psi_{0}(s)=\log s$ if $d=2$. Note that $W\left(1, \psi_{0}\right)(s)=s^{-\frac{d}{2}}$ and thus, by the variation of constants formula, $\phi$ can be written as

$$
\phi(s)=c_{0}+c_{1} \psi_{0}(s)-\int_{0}^{s} \psi_{0}(t) t^{\frac{d}{2}-1} h(t) d t+\psi_{0}(s) \int_{0}^{s} t^{\frac{d}{2}-1} h(t) d t
$$

for some constants $c_{0}, c_{1} \in \mathbb{R}$. Since $\phi \in C([0, \infty))$, we must have $c_{1}=0$ and therefore,

$$
\phi^{\prime}(s)=\psi_{0}^{\prime}(s) \int_{0}^{s} t^{\frac{d}{2}-1} h(t) d t=s^{-\frac{d}{2}} \int_{0}^{s} t^{\frac{d}{2}-1} h(t) d t .
$$

Consequently, by de l'Hôpital's rule,

$$
\lim _{s \rightarrow 0+} \phi^{\prime}(s)=\lim _{s \rightarrow 0+} \frac{\int_{0}^{s} t^{\frac{d}{2}-1} h(t) d t}{s^{\frac{d}{2}}}=\lim _{s \rightarrow 0+} \frac{s^{\frac{d}{2}-1} h(s)}{\frac{d}{2} s^{\frac{d}{2}-1}}=\frac{2}{d} h(0),
$$

and we see that $\phi \in C^{1}([0, \infty))$. Furthermore,

$$
\phi^{\prime \prime}(s)=s^{-1} h(s)-\frac{d}{2} s^{-\frac{d}{2}-1} \int_{0}^{s} t^{\frac{d}{2}-1} h(t) d t=s^{-1} h(s)-\frac{d}{2} s^{-1} \phi^{\prime}(s)
$$

and thus,

$$
\lim _{s \rightarrow 0+}\left[s \phi^{\prime \prime}(s)\right]=\lim _{s \rightarrow 0+}\left[h(s)-\frac{d}{2} \phi^{\prime}(s)\right]=0 .
$$

By definition, $f(x)=\phi\left(|x|^{2}\right)$ and thus,

$$
\partial_{j} \partial_{k} f(x)=4 x_{j} x_{k} \phi^{\prime \prime}\left(|x|^{2}\right)+2 \phi^{\prime}\left(|x|^{2}\right) \delta_{j k} .
$$

This implies that

$$
\lim _{x \rightarrow 0} \partial_{j} \partial_{k} f(x)=2 \phi^{\prime}(0) \delta_{j k}
$$

since

$$
\left|x_{j} x_{k} \phi^{\prime \prime}\left(|x|^{2}\right)\right| \leq|x|^{2}\left|\phi^{\prime \prime}\left(|x|^{2}\right)\right| \rightarrow 0
$$

as $|x| \rightarrow 0$. Consequently, $f \in C^{2}\left(\mathbb{R}^{d}\right)$. 


\section{Appendix B. Construction of a fundamental system}

Lemma B.1. Let $\lambda>0$ and $V \in C^{\infty}([0, \infty))$. Furthermore, suppose that for any $k \in \mathbb{N}_{0}$ there exists a $C_{k}>0$ such that $\left|V^{(k)}(r)\right| \leq C_{k}\langle r\rangle^{-2-k}$ for all $r \geq 0$. Then the equation

$$
\phi^{\prime \prime}(r)-\frac{(d-1)(d-3)}{4 r^{2}} \phi(r)-V(r) \phi(r)-\lambda^{2} \phi(r)=0
$$

has fundamental systems $\left\{\phi_{0}, \psi_{0}\right\}$ on $\left(0, \frac{1}{2}\right]$ and $\left\{\phi_{\infty}, \psi_{\infty}\right\}$ on $\left[\frac{1}{4}, \infty\right)$, respectively, of the form

$$
\begin{aligned}
\phi_{0}(r) & =r^{\frac{d-1}{2}}\left[1+a_{0}(r)\right], & \psi_{0}(r) & = \begin{cases}r^{\frac{1}{2}} \log r\left[1+b_{0}(r)\right], & d=2, \\
-\frac{1}{d-2} r^{-\frac{d-3}{2}}\left[1+b_{0}(r)\right], & d \neq 2,\end{cases} \\
\phi_{\infty}(r) & =e^{-\lambda r}\left[1+a_{\infty}(r)\right], & \psi_{\infty}(r) & =\frac{1}{2 \lambda} e^{\lambda r}\left[1+b_{\infty}(r)\right] .
\end{aligned}
$$

For any $k \in \mathbb{N}_{0}$ there exists a $C_{k}>0$ such that

$$
\begin{aligned}
\left|a_{0}^{(k)}(r)\right| & \leq C_{k} r^{1-k}, & \text { for all } r \in\left(0, \frac{1}{2}\right], \\
\left|b_{0}^{(k)}(r)\right| & \leq C_{k} \begin{cases}|\log r|^{-1} r^{-k}, & d=2, \\
r^{1-k}, & d \neq 2,\end{cases} & \text { for all } r \in\left(0, \frac{1}{2}\right], \\
\left|a_{\infty}^{(k)}(r)\right|+\left|b_{\infty}^{(k)}(r)\right| & \leq C_{k} r^{-1-k}, & \text { for all } r \geq \frac{1}{4} .
\end{aligned}
$$

Proof. We start with the construction of the solution $\phi_{0}$. Note that the equation

$$
f^{\prime \prime}(r)-\frac{(d-1)(d-3)}{4 r^{2}} f(r)=0
$$

has the fundamental system $\left\{f_{0}, g_{0}\right\}$, given by

$$
f_{0}(r)=r^{\frac{d-1}{2}}, \quad g_{0}(r)= \begin{cases}r^{\frac{1}{2}} \log r, & \text { if } d=2, \\ -\frac{1}{d-2} r^{-\frac{d-3}{2}}, & \text { if } d \neq 2,\end{cases}
$$

and $W\left(f_{0}, g_{0}\right)=1$. Thus, in view of the variation of constants formula, $\phi_{0}$ is supposed to solve the equation

$$
\phi_{0}(r)=f_{0}(r)-f_{0}(r) \int_{0}^{r} g_{0}(s)\left[V(s)+\lambda^{2}\right] \phi_{0}(s) d s+g_{0}(r) \int_{0}^{r} f_{0}(s)\left[V(s)+\lambda^{2}\right] \phi_{0}(s) d s .
$$

We rewrite this equation in terms of the auxiliary function $h$, defined by $\phi_{0}=f_{0} h$. This yields the Volterra equation

$$
h(r)=1+\int_{0}^{r} K(r, s) h(s) d s
$$

with the kernel

$$
K(r, s):=\left[\frac{g_{0}(r)}{f_{0}(r)} f_{0}(s)^{2}-f_{0}(s) g_{0}(s)\right]\left[V(s)+\lambda^{2}\right] .
$$

If $d \geq 3$ we have the bound

$$
|K(r, s)| \lesssim r^{-d+2} s^{d-1}+s \lesssim s
$$

\footnotetext{
${ }^{3}$ The bounds on the error functions $a_{0}, b_{0}$ are not optimal but simple to work with and sufficient for our purposes.
} 
for all $0 \leq s \leq r \leq \frac{1}{2}$. If $d=2$, we fix an arbitrary $\delta \in(0,1)$ and estimate

$$
|K(r, s)| \lesssim|\log r| s+s|\log s| \lesssim s^{1-\delta}
$$

for all $0 \leq s \leq r \leq \frac{1}{2}$. Consequently,

$$
\int_{0}^{\frac{1}{2}} \sup _{r \in\left[s, \frac{1}{2}\right]}|K(r, s)| d s \lesssim 1
$$

and the standard existence result for Volterra equations (see e.g. [60], Lemma 2.4) yields the existence of a solution $h \in L^{\infty}\left(0, \frac{1}{2}\right)$ to Eq. (B.2), satisfying the bound

$$
|h(r)-1| \lesssim \int_{0}^{r}|K(r, s)||h(s)| d s \lesssim\|h\|_{L^{\infty}\left(0, \frac{1}{2}\right)} \int_{0}^{r} s^{1-\delta} d s \lesssim r^{2-\delta}
$$

for all $r \in\left[0, \frac{1}{2}\right]$. This proves the existence of $\phi_{0}(r)=f_{0}(r) h(r)=f_{0}(r)\left[1+a_{0}(r)\right]$, with the bound $\left|a_{0}(r)\right| \lesssim r^{2-\delta} \lesssim r$ for all $r \in\left[0, \frac{1}{2}\right]$.

Next, we turn to the derivative bounds on $a_{0}$. For any $j \in \mathbb{N}_{0}$ we have the bound $\left|\partial_{r}^{j} K(r, s)\right| \lesssim r^{-j} s^{1-\delta} \lesssim r^{-j}$ for all $0<r \leq s \leq \frac{1}{2}$. If we set $\kappa_{j}(r):=\left.\partial_{r}^{j} K(r, s)\right|_{s=r}$, then, for $j, k \in \mathbb{N}_{0}$, we have $\left|\kappa_{j}^{(k)}(r)\right| \lesssim r^{-j-k}$ for all $r \in\left(0, \frac{1}{2}\right]$. In terms of $a_{0}$, Eq. (B.2) reads

$$
a_{0}(r)=\int_{0}^{r} K(r, s) d s+\int_{0}^{r} K(r, s) a_{0}(s) d s .
$$

Thus, for $k \in \mathbb{N}$, we obtain

$$
a_{0}^{(k)}(r)=\kappa_{0}^{(k-1)}(r)+\sum_{j=0}^{k-1}\left(\kappa_{j} a_{0}\right)^{(k-1-j)}(r)+\int_{0}^{r} \partial_{r}^{k} K(r, s) a_{0}(s) d s .
$$

Inductively, we find

$$
\left|a_{0}^{(k)}(r)\right| \lesssim r^{1-k}+r^{2-k} \lesssim r^{1-k}
$$

for all $r \in\left(0, \frac{1}{2}\right]$, which is the desired bound.

The singular solution $\psi_{0}$ is constructed via the reduction formula. Since $\phi_{0}(r)=r^{\frac{d-1}{2}}[1+$ $O(r)]$, there exists an $r_{0} \in\left(0, \frac{1}{2}\right]$ such that $\phi_{0}(r)>0$ for all $r \in\left(0, r_{0}\right]$. Consequently,

$$
\psi_{0}(r):=-\phi_{0}(r) \int_{r}^{r_{0}} \phi_{0}(s)^{-2} d s
$$

is well-defined for all $r \in\left(0, r_{0}\right]$ and provides a solution to Eq. (B.1) on $\left(0, r_{0}\right]$. We define the function $b_{0}$ on $\left(0, r_{0}\right]$ by $\psi_{0}(r)=g_{0}(r)\left[1+b_{0}(r)\right]$, i.e.,

$$
b_{0}(r):=-\frac{f_{0}(r)\left[1+a_{0}(r)\right]}{g_{0}(r)} \int_{r}^{r_{0}} f_{0}(s)^{-2}\left[1+a_{0}(s)\right]^{-2} d s-1 .
$$

Observe that

$$
\left(\frac{g_{0}}{f_{0}}\right)^{\prime}=\frac{f_{0} g_{0}^{\prime}-f_{0}^{\prime} g_{0}}{f_{0}^{2}}=\frac{W\left(f_{0}, g_{0}\right)}{f_{0}^{2}}=f_{0}^{-2}
$$

and thus,

$$
-\frac{f_{0}(r)}{g_{0}(r)} \int_{r}^{r_{0}} f_{0}(s)^{-2} d s=1-c_{0} \frac{f_{0}(r)}{g_{0}(r)}, \quad c_{0}:=\frac{g_{0}\left(r_{0}\right)}{f_{0}\left(r_{0}\right)} .
$$


Consequently,

$$
b_{0}(r)=a_{0}(r)-c_{0} \frac{f_{0}(r)}{g_{0}(r)}\left[1+a_{0}(r)\right]-\frac{f_{0}(r)}{g_{0}(r)}\left[1+a_{0}(r)\right] \int_{r}^{r_{0}} f_{0}(s)^{-2}\left[\left(1+a_{0}(s)\right)^{-2}-1\right] d s,
$$

and in the case $d \geq 3$ we obtain

$$
\left|b_{0}(r)\right| \lesssim r+r^{d-2}+r^{d-2} \int_{r}^{r_{0}} s^{-d+1} s^{2-\delta} d s \lesssim r+r^{d-2}+r^{2-\delta} \lesssim r
$$

for all $r \in\left[0, r_{0}\right]$. In the case $d=2$ we have the weaker bound

$$
\left|b_{0}(r)\right| \lesssim r+|\log r|^{-1}+|\log r|^{-1} \int_{r}^{r_{0}} s^{-1} s^{2-\delta} d s \lesssim|\log r|^{-1}
$$

for all $r \in\left(0, r_{0}\right]$. The derivative bounds on $b_{0}$ follow directly by differentiating the explicit formula for $b_{0}$. By solving an initial value problem with data at $r=r_{0}$, we extend the solution $\psi_{0}$ to $\left(0, \frac{1}{2}\right]$ and clearly, $\psi_{0} \in C^{\infty}\left(\left(0, \frac{1}{2}\right]\right)$ since the coefficients of Eq. (B.1) are smooth on $(0, \infty)$.

The solution $\phi_{\infty}$ is constructed by a similar procedure. This time we treat the term $-\frac{(d-1)(d-3)}{4 r^{2}} \phi(r)$ perturbatively since it is negligible for large $r$. That is to say, we first note that the equation

$$
f^{\prime \prime}(r)-\lambda^{2} f(r)=0
$$

has the fundamental system $\left\{f_{\infty}, g_{\infty}\right\}$, where $f_{\infty}(r)=e^{-\lambda r}$ and $g_{\infty}(r)=\frac{1}{2 \lambda} e^{\lambda r}$. Consequently, we write $\phi_{\infty}=f_{\infty} h$ and consider the Volterra equation

$$
h(r)=1+\int_{r}^{\infty} K(r, s) h(s) d s
$$

with the kernel

$$
K(r, s):=\left[f_{\infty}(s) g_{\infty}(s)-\frac{g_{\infty}(r)}{f_{\infty}(r)} f_{\infty}(s)^{2}\right]\left[\frac{(d-1)(d-3)}{4 s^{2}}+V(s)\right] .
$$

We estimate

$$
|K(r, s)| \lesssim\left(1+e^{2 \lambda r} e^{-2 \lambda s}\right) s^{-2} \lesssim s^{-2}
$$

for all $\frac{1}{4} \leq r \leq s$, which yields

$$
\int_{\frac{1}{4}}^{\infty} \sup _{r \in\left[\frac{1}{4}, s\right]}|K(r, s)| d s \lesssim \int_{\frac{1}{4}}^{\infty} s^{-2} d s \lesssim 1
$$

The Volterra theorem (see e.g. [60], Lemma 2.4) then implies the existence of a solution $h \in L^{\infty}\left(\frac{1}{4}, \infty\right)$. Furthermore,

$$
|h(r)-1| \leq \int_{r}^{\infty}|K(r, s)||h(s)| d s \lesssim\|h\|_{L^{\infty}\left(\frac{1}{4}, \infty\right)} \int_{r}^{\infty} s^{-2} d s \lesssim r^{-1}
$$

for all $r \geq \frac{1}{4}$ and thus, $\phi_{\infty}(r)=f_{\infty}(r)\left[1+a_{\infty}(r)\right]$ with $\left|a_{\infty}(r)\right| \lesssim r^{-1}$ for all $r \geq \frac{1}{4}$. 
For the bounds on the derivatives of $a_{\infty}$, we rewrite the Volterra equation for $a_{\infty}=h-1$ as

$$
\begin{aligned}
a_{\infty}(r) & =\int_{r}^{\infty} K(r, s) d s+\int_{r}^{\infty} K(r, s) a_{\infty}(s) d s \\
& =\int_{0}^{\infty} K(r, s+r) d s+\int_{0}^{\infty} K(r, s+r) a_{\infty}(s+r) d s .
\end{aligned}
$$

Note that

$$
\begin{aligned}
K(r, s+r) & =\frac{1}{2 \lambda}\left(1-\frac{e^{\lambda r}}{e^{-\lambda r}} e^{-2 \lambda(s+r)}\right)\left[\frac{(d-1)(d-3)}{4(s+r)^{2}}+V(s+r)\right] \\
& =\frac{1}{2 \lambda}\left(1-e^{-2 \lambda s}\right)\left[\frac{(d-1)(d-3)}{4(s+r)^{2}}+V(s+r)\right]
\end{aligned}
$$

and thus, for $j \in \mathbb{N}_{0}$,

$$
\left|\partial_{r}^{j} K(r, s+r)\right| \lesssim(s+r)^{-2-j}
$$

for all $\frac{1}{4} \leq r \leq s$. Now let $k \in \mathbb{N}$ and assume that for any $j \in \mathbb{N}_{0}$ with $j \leq k-1$, we have $\left|a_{\infty}^{(j)}(r)\right| \lesssim r^{-1-j}$ for all $r \geq \frac{1}{4}$. Then we obtain

$$
\begin{aligned}
a_{\infty}^{(k)}(r) & =\int_{0}^{\infty} \partial_{r}^{k} K(r, s+r) d s+\int_{0}^{\infty} \partial_{r}^{k}\left[K(r, s+r) a_{\infty}(s+r)\right] d s \\
& =O\left(r^{-1-k}\right)+\int_{r}^{\infty} K(r, s) a_{\infty}^{(k)}(s) d s
\end{aligned}
$$

and thus, $a_{k}(r):=r^{1+k} a_{\infty}^{(k)}(r)$ satisfies the Volterra equation

$$
a_{k}(r)=O\left(r^{0}\right)+\int_{r}^{\infty} K(r, s) r^{1+k} s^{-1-k} a_{k}(s) d s .
$$

Since

$$
\left|K(r, s) r^{1+k} s^{-1-k}\right| \lesssim s^{-2}
$$

for all $\frac{1}{4} \leq r \leq s$, a Volterra iteration yields $a_{k} \in L^{\infty}\left(\frac{1}{4}, \infty\right)$ and we obtain

$$
\left|a_{\infty}^{(k)}(r)\right|=\left|r^{-1-k} a_{k}(r)\right| \leq r^{-1-k}\left\|a_{k}\right\|_{L^{\infty}\left(\frac{1}{4}, \infty\right)} \lesssim r^{-1-k}
$$

for all $r \geq \frac{1}{4}$. Consequently, the stated bounds on the derivatives of $a_{\infty}$ follow inductively.

Finally, for the growing solution $\psi_{\infty}$, we note that there exists an $r_{1} \geq \frac{1}{4}$ such that $\phi_{\infty}(r)>0$ for all $r \geq r_{1}$ and set

$$
\psi_{\infty}(r):=\phi_{\infty}(r) \int_{r_{1}}^{r} \phi_{\infty}(s)^{-2} d s .
$$

Then $\psi_{\infty}$ solves Eq. (B.1) on $\left[r_{1}, \infty\right)$ and the function $b_{\infty}$, defined by $\psi_{\infty}=g_{\infty}\left(1+b_{\infty}\right)$, is given explicitly by

$$
b_{\infty}(r)=\frac{f_{\infty}(r)\left[1+a_{\infty}(r)\right]}{g_{\infty}(r)} \int_{r_{1}}^{r} f_{\infty}(s)^{-2}\left[1+a_{\infty}(s)\right]^{-2} d s-1 .
$$

As before,

$$
\frac{f_{\infty}(r)}{g_{\infty}(r)} \int_{r_{1}}^{r} f_{\infty}(s)^{-2} d s=1-c_{1} \frac{f_{\infty}(r)}{g_{\infty}(r)}, \quad c_{1}:=\frac{g_{\infty}\left(r_{1}\right)}{f_{\infty}\left(r_{1}\right)},
$$


and thus,

$$
b_{\infty}(r)=a_{\infty}(r)-c_{1} \frac{f_{\infty}(r)}{g_{\infty}(r)}\left[1+a_{\infty}(r)\right]+\frac{f_{\infty}(r)}{g_{\infty}(r)}\left[1+a_{\infty}(r)\right] \int_{r_{1}}^{r} f_{\infty}(s)^{-2}\left[\left(1+a_{\infty}(s)\right)^{-2}-1\right] d s .
$$

This yields the bound

$$
\left|b_{\infty}(r)\right| \lesssim r^{-1}+e^{-2 \lambda r}+e^{-2 \lambda r} \int_{r_{1}}^{r} e^{2 \lambda s} s^{-1} d s \lesssim r^{-1}
$$

for all $r \geq \frac{1}{4}$. The bounds on the derivatives of $b_{\infty}$ follow in a straightforward manner by differentiating the explicit expression for $b_{\infty}$. By solving an initial value problem, the solution $\psi_{\infty}$ smoothly extends to all of $\left[\frac{1}{4}, \infty\right)$.

\section{ApPEndix C. Numerics}

C.1. Numerical construction of the soliton profile. We would like to obtain a radial solution to

$$
\Delta_{\mathbb{R}^{2}} Q-Q+Q^{3}=0 .
$$

That is to say, we need to solve the radial equation

$$
f^{\prime \prime}(r)+\frac{1}{r} f^{\prime}(r)-f(r)+f(r)^{3}=0
$$

for $r \geq 0$. Asymptotically, the nonlinearity is negligible and thus, we expect the behavior $f(r) \simeq 1$ as $r \rightarrow 0+$ and $f(r) \simeq r^{-\frac{1}{2}} e^{-r}$ as $r \rightarrow \infty$. We encode the expected asymptotics in the definition of the new variable $g$, given by

$$
f(r)=:(1+r)^{-\frac{1}{2}} e^{-r} g\left(\frac{r-1}{r+1}\right) .
$$

In terms of $g$ and $x:=\frac{r-1}{r+1}$, Eq. (C.1) reads

$$
\mathcal{R}(g):=g^{\prime \prime}(x)+\frac{3 x^{2}-6 x-5}{(1-x)^{2}(1+x)} g^{\prime}(x)-\frac{3(3-x)}{4(1-x)^{2}(1+x)} g(x)+\frac{2}{(1-x)^{3}} e^{-2 \frac{1+x}{1-x}} g(x)^{3}=0
$$

for $x \in[-1,1)$. We compactify the problem (C.2) by allowing $x \in[-1,1]$. Evidently, the endpoints $x= \pm 1$ are singular and this yields the regularity conditions

$$
4 g^{\prime}(-1)-3 g(-1)=16 g^{\prime}(1)+3 g(1)=16 g^{\prime \prime}(1)-5 g^{\prime}(1)-3 g(1)=0 .
$$

Note that these conditions are determined by the linear part of the equation since the coefficient of $g(x)^{3}$ is not singular at $x= \pm 1$. We solve Eq. (C.2) by a Chebyshev pseudospectral method. To this end, we use the basis functions $\phi_{n}:[-1,1] \rightarrow \mathbb{R}, n \in \mathbb{N}_{0}$,

$$
\phi_{n}(x):=T_{n}(x)+a_{0, n}+a_{1, n} x+a_{2, n} x^{2},
$$

where $T_{n}$ are the standard Chebyshev polynomials and $a_{j, n}$ are chosen in such a way that each $\phi_{n}$ satisfies the regularity conditions Eq. (C.3). Note that this leads to $\phi_{0}=\phi_{1}=\phi_{2}=0$. Then we numerically solve the root finding problem

$$
\mathcal{R}\left(\sum_{n=3}^{25} \beta_{n} \phi_{n}\right)\left(x_{k}\right)=0
$$


for $k=0,1,2, \ldots, 22$, and $x_{k} \in[-1,1]$ some collocation points. The expansion coefficients $\left(\beta_{n}\right)_{n=3}^{25}$ are given in Table C.1.

TABLE C.1. Expansion coefficients for the approximate soliton profile

\begin{tabular}{|c|c|c|c|c|c|c|}
\hline$n$ & 3 & 4 & 5 & 6 & 7 & 8 \\
$\beta_{n}$ & $-\frac{2542}{141001}$ & $\frac{8061}{72860}$ & $\frac{23}{25643}$ & $-\frac{17127}{731900}$ & $-\frac{113}{61446}$ & $\frac{407}{88530}$ \\
\hline$n$ & 9 & 10 & 11 & 12 & 13 & 14 \\
$\beta_{n}$ & $\frac{80}{79969}$ & $-\frac{195}{296276}$ & $-\frac{167}{607101}$ & $\frac{3}{91531}$ & $\frac{3}{109289}$ & $\frac{1}{42237921}$ \\
\hline$n$ & 15 & 16 & 17 & 18 & 19 & 20 \\
$\beta_{n}$ & $\frac{1}{163112}$ & $\frac{1}{171418}$ & $\frac{1}{1839428}$ & $-\frac{1}{412985}$ & $-\frac{1}{693490}$ & $-\frac{1}{3459389}$ \\
\hline$n$ & 21 & 22 & 23 & 24 & 25 & \\
$\beta_{n}$ & $\frac{1}{5641102}$ & $\frac{1}{2626342}$ & $\frac{1}{45286837}$ & $\frac{1}{10226264}$ & $-\frac{1}{9836273}$ & \\
\hline
\end{tabular}

C.2. Numerical construction of $\widehat{Q}_{1}$. The goal is to numerically construct the unique (radial) solution $\widehat{Q}_{1}$ to the equation

$$
\mathcal{L}_{+} \widehat{Q}_{1}=-2 Q_{\mathbb{R}^{2}}-r^{2} Q_{\mathbb{R}^{2}}^{3} .
$$

Recall that $S_{0}(x)=x^{j} \partial_{j} Q_{\mathbb{R}^{2}}(x)+Q_{\mathbb{R}^{2}}(x)$ satisfies $\mathcal{L}_{+} S_{0}=-2 Q_{\mathbb{R}^{2}}$. Consequently, it suffices to solve $\mathcal{L}_{+} S_{1}=-r^{2} Q_{\mathbb{R}^{2}}^{3}$ because then, $\widehat{Q}_{1}=S_{0}+S_{1}$. In other words, we need to solve the radial equation

$$
f^{\prime \prime}(r)+\frac{1}{r} f^{\prime}(r)-f(r)+3 f_{0}(r)^{2} f(r)=r^{2} f_{0}(r)^{3}
$$

where $f_{0}(r)=Q_{\mathbb{R}^{2}}\left(r e_{1}\right)$. Again, we introduce the auxiliary variable $g$, defined by

$$
f(r)=(1+r)^{-\frac{1}{2}} e^{-r} g\left(\frac{r-1}{r+1}\right),
$$

which transforms Eq. (C.4) into

$$
\begin{aligned}
g^{\prime \prime}(x) & +\frac{3 x^{2}-6 x-5}{(1-x)^{2}(1+x)} g^{\prime}(x)-\frac{3(3-x)}{4(1-x)^{2}(1+x)} g(x)+\frac{6}{(1-x)^{3}} e^{-2 \frac{1+x}{1-x}} g_{0}(x)^{2} g(x) \\
& =2 \frac{(1+x)^{2}}{(1-x)^{5}} e^{-2 \frac{1+x}{1-x}} g_{0}(x)^{3},
\end{aligned}
$$

where $x=\frac{r-1}{r+1}$ and $g_{0}$ is given by

$$
f_{0}(r)=(1+r)^{-\frac{1}{2}} e^{-r} g_{0}\left(\frac{r-1}{r+1}\right) .
$$

We replace $g_{0}$ by the approximation obtained in Section C.1 and solve Eq. (C.5) by a Chebyshev pseudospectral method with the basis functions $\phi_{n}$ from above. This yields an approximate solution of the form $\sum_{n=3}^{40} \gamma_{n} \phi_{n}$ with the coefficients $\left(\gamma_{n}\right)_{n=3}^{40}$ given in Table C.2.

With the numerical approximations to the functions $Q_{\mathbb{R}^{2}}$ and $\widehat{Q}_{1}$ at hand, it is straightforward to compute (an approximation to) the constant $b_{1}$ from Section 6.2. By numerical integration we find $\frac{b_{1}}{2 \pi} \approx 7.39$. 
TABle C.2. Expansion coefficients for approximation to $S_{1}$

\begin{tabular}{|c|c|c|c|c|c|c|c|c|}
\hline$n$ & 3 & 4 & 5 & 6 & 7 & 8 & 9 & 10 \\
$\gamma_{n}$ & $\frac{54973}{96387}$ & $-\frac{3088}{102021}$ & $-\frac{11563}{65730}$ & $-\frac{622}{123831}$ & $\frac{935}{19694}$ & $\frac{715}{80273}$ & $-\frac{972}{107461}$ & $-\frac{245}{66869}$ \\
\hline$n$ & 11 & 12 & 13 & 14 & 15 & 16 & 17 & 18 \\
$\gamma_{n}$ & $\frac{43}{75440}$ & $\frac{6}{13097}$ & $\frac{7}{79466}$ & $\frac{23}{138473}$ & $\frac{10}{87071}$ & $-\frac{1}{41044}$ & $-\frac{7}{100544}$ & $-\frac{3}{79736}$ \\
\hline$n$ & 19 & 20 & 21 & 22 & 23 & 24 & 25 & 26 \\
$\gamma_{n}$ & $-\frac{1}{247350}$ & $\frac{1}{98688}$ & $\frac{1}{104302}$ & $\frac{1}{181864}$ & $\frac{1}{1748151}$ & $-\frac{1}{795239}$ & $-\frac{1}{519650}$ & $-\frac{1}{1141942}$ \\
\hline$n$ & 27 & 28 & 29 & 30 & 31 & 32 & 33 & 34 \\
$\gamma_{n}$ & $-\frac{1}{2632970}$ & $\frac{1}{3481458}$ & $\frac{1}{4334802}$ & $\frac{1}{3856839}$ & $\frac{1}{14342913}$ & $-\frac{1}{142634956}$ & $-\frac{1}{42463795}$ & $-\frac{1}{12658667}$ \\
\hline$n$ & 35 & 36 & 37 & 38 & 39 & 40 & & \\
$\gamma_{n}$ & $\frac{1}{45132528}$ & $-\frac{1}{14926347}$ & $\frac{1}{15529718}$ & $-\frac{1}{15419336}$ & $\frac{1}{13135736}$ & $-\frac{1}{36714512}$ & & \\
\hline
\end{tabular}

\section{REFERENCES}

[1] Jean-Philippe Anker and Vittoria Pierfelice. Nonlinear Schrödinger equation on real hyperbolic spaces. Ann. Inst. H. Poincaré Anal. Non Linéaire, 26(5):1853-1869, 2009.

[2] V. Banica. The nonlinear Schrödinger equation on hyperbolic space. Comm. Partial Differential Equations, 32(10-12):1643-1677, 2007.

[3] Valeria Banica and Thomas Duyckaerts. Weighted Strichartz estimates for radial Schrödinger equation on noncompact manifolds. Dyn. Partial Differ. Equ., 4(4):335-359, 2007.

[4] Valeria Banica and Thomas Duyckaerts. Global existence, scattering and blow-up for the focusing NLS on the hyperbolic space. Dyn. Partial Differ. Equ., 12(1):53-96, 2015.

[5] Marius Beceanu. A centre-stable manifold for the focussing cubic NLS in $\mathbb{R}^{1+3}$. Comm. Math. Phys., 280(1):145-205, 2008.

[6] Marius Beceanu. A critical center-stable manifold for Schrödinger's equation in three dimensions. Comm. Pure Appl. Math., 65(4):431-507, 2012.

[7] H. Berestycki and P.-L. Lions. Nonlinear scalar field equations. I. Existence of a ground state. Arch. Rational Mech. Anal., 82(4):313-345, 1983.

[8] Henri Berestycki and Thierry Cazenave. Instabilité des états stationnaires dans les équations de Schrödinger et de Klein-Gordon non linéaires. C. R. Acad. Sci. Paris Sér. I Math., 293(9):489-492, 1981.

[9] Henri Berestycki, Thierry Gallouët, and Otared Kavian. équations de champs scalaires euclidiens non linéaires dans le plan. C. R. Acad. Sci. Paris Sér. I Math., 297(5):307-310, 1983.

[10] David Borthwick and Jeremy L. Marzuola. Dispersive estimates for scalar and matrix Schrödinger operators on $\mathbb{H}^{n+1}$. Math. Phys. Anal. Geom., 18(1):Art. 22, 26, 2015.

[11] Jean-Marc Bouclet. Low frequency estimates and local energy decay for asymptotically euclidean laplacians. Communications in Partial Differential Equations, 36(7):1239-1286, 2011.

[12] Thomas Boulenger. Blow-up solutions for the 2-dimensional critical Schrödinger equation on a riemannian manifold. PhD thesis, Université Paris Sud-Paris XI, 2012.

[13] V. S. Buslaev and G. S. Perelman. On the stability of solitary waves for nonlinear Schrödinger equations. In Nonlinear evolution equations, volume 164 of Amer. Math. Soc. Transl. Ser. 2, pages 75-98. Amer. Math. Soc., Providence, RI, 1995.

[14] Vladimir S. Buslaev and Catherine Sulem. On asymptotic stability of solitary waves for nonlinear Schrödinger equations. Ann. Inst. H. Poincaré Anal. Non Linéaire, 20(3):419-475, 2003.

[15] T. Cazenave and P.-L. Lions. Orbital stability of standing waves for some nonlinear Schrödinger equations. Comm. Math. Phys., 85(4):549-561, 1982.

[16] Shu-Ming Chang, Stephen Gustafson, Kenji Nakanishi, and Tai-Peng Tsai. Spectra of linearized operators for NLS solitary waves. SIAM J. Math. Anal., 39(4):1070-1111, 2007/08. 
[17] Xi Chen. Resolvent and spectral measure on non-trapping asymptotically hyperbolic manifolds iii: Global-in-time strichartz estimates without loss. In Annales de l'Institut Henri Poincaré C, Analyse non linéaire, volume 35, pages 803-829. Elsevier, 2018.

[18] Xi Chen and Andrew Hassell. Resolvent and spectral measure on non-trapping asymptotically hyperbolic manifolds ii: spectral measure, restriction theorem, spectral multiplier. arXiv preprint arXiv:1412.442\%, 2014.

[19] Xi Chen and Andrew Hassell. Resolvent and spectral measure on non-trapping asymptotically hyperbolic manifolds i: Resolvent construction at high energy. Communications in Partial Differential Equations, 41(3):515-578, 2016.

[20] Hans Christianson, Jeremy Marzuola, Jason Metcalfe, and Michael Taylor. Nonlinear bound states on weakly homogeneous spaces. Comm. Partial Differential Equations, 39(1):34-97, 2014.

[21] Hans Christianson and Jeremy L. Marzuola. Existence and stability of solitons for the nonlinear Schrödinger equation on hyperbolic space. Nonlinearity, 23(1):89-106, 2010.

[22] Charles V. Coffman. Uniqueness of the ground state solution for $\Delta u-u+u^{3}=0$ and a variational characterization of other solutions. Arch. Rational Mech. Anal., 46:81-95, 1972.

[23] S. Coleman, V. Glaser, and A. Martin. Action minima among solutions to a class of Euclidean scalar field equations. Comm. Math. Phys., 58(2):211-221, 1978.

[24] Ovidiu Costin, Min Huang, and Wilhelm Schlag. On the spectral properties of $L_{ \pm}$in three dimensions. Nonlinearity, 25(1):125-164, 2012.

[25] Scipio Cuccagna. Stabilization of solutions to nonlinear Schrödinger equations. Comm. Pure Appl. Math., 54(9):1110-1145, 2001.

[26] Scipio Cuccagna. On asymptotic stability of ground states of NLS. Rev. Math. Phys., 15(8):877-903, 2003.

[27] Scipio Cuccagna. On asymptotic stability of ground states of nls. Reviews in Mathematical Physics, 15(08):877-903, 2003.

[28] Scipio Cuccagna. The Hamiltonian structure of the nonlinear Schrödinger equation and the asymptotic stability of its ground states. Comm. Math. Phys., 305(2):279-331, 2011.

[29] Scipio Cuccagna and Tetsu Mizumachi. On asymptotic stability in energy space of ground states for nonlinear Schrödinger equations. Comm. Math. Phys., 284(1):51-77, 2008.

[30] Scipio Cuccagna, Dmitry Pelinovsky, and Vitali Vougalter. Spectra of positive and negative energies in the linearized NLS problem. Comm. Pure Appl. Math., 58(1):1-29, 2005.

[31] Laurent Demanet and Wilhelm Schlag. Numerical verification of a gap condition for a linearized nonlinear Schrödinger equation. Nonlinearity, 19(4):829-852, 2006.

$[32]$ D. E. Edmunds and W. D. Evans. Spectral theory and differential operators. Oxford Mathematical Monographs. The Clarendon Press, Oxford University Press, New York, 1987. Oxford Science Publications.

[33] María Jesús Esteban. Existence d'une infinité d'ondes solitaires pour des équations de champs non linéaires dans le plan. Ann. Fac. Sci. Toulouse Math. (5), 2(3-4):181-191, 1980.

[34] B. Gidas, Wei Ming Ni, and L. Nirenberg. Symmetry of positive solutions of nonlinear elliptic equations in $\mathbf{R}^{n}$. In Mathematical analysis and applications, Part A, volume 7 of Adv. in Math. Suppl. Stud., pages 369-402. Academic Press, New York-London, 1981.

[35] Manoussos Grillakis. Linearized instability for nonlinear Schrödinger and Klein-Gordon equations. Comm. Pure Appl. Math., 41(6):747-774, 1988.

[36] Manoussos Grillakis, Jalal Shatah, and Walter Strauss. Stability theory of solitary waves in the presence of symmetry. I. J. Funct. Anal., 74(1):160-197, 1987.

[37] Manoussos Grillakis, Jalal Shatah, and Walter Strauss. Stability theory of solitary waves in the presence of symmetry. II. J. Funct. Anal., 94(2):308-348, 1990.

[38] K. Gustafson and J. Weidmann. On the essential spectrum. J. Math. Anal. Appl., 25:121-127, 1969.

[39] Stephen Gustafson, Kenji Nakanishi, and Tai-Peng Tsai. Asymptotic stability and completeness in the energy space for nonlinear schrödinger equations with small solitary waves. International Mathematics Research Notices, 2004(66):3559-3584, 2004.

[40] Andrew Hassell and Junyong Zhang. Global-in-time strichartz estimates on nontrapping, asymptotically conic manifolds. Analysis \& PDE, 9(1):151-192, 2016. 
[41] Lars Hörmander. The analysis of linear partial differential operators. I. Classics in Mathematics. Springer-Verlag, Berlin, 2003. Distribution theory and Fourier analysis, Reprint of the second (1990) edition [Springer, Berlin; MR1065993 (91m:35001a)].

[42] Dirk Hundertmark and Young-Ran Lee. Exponential decay of eigenfunctions and generalized eigenfunctions of a non-self-adjoint matrix Schrödinger operator related to NLS. Bull. Lond. Math. Soc., 39(5):709-720, 2007.

[43] Tosio Kato. Perturbation theory for linear operators. Classics in Mathematics. Springer-Verlag, Berlin, 1995. Reprint of the 1980 edition.

[44] E Kirr, PG Kevrekidis, and DE Pelinovsky. Symmetry-breaking bifurcation in the nonlinear schrödinger equation with symmetric potentials. Communications in mathematical physics, 308(3):795-844, 2011.

[45] EW Kirr, PG Kevrekidis, E Shlizerman, and Michael I Weinstein. Symmetry-breaking bifurcation in nonlinear schrödinger/gross-pitaevskii equations. SIAM Journal on Mathematical Analysis, 40(2):566604, 2008.

[46] J. Krieger and W. Schlag. Stable manifolds for all monic supercritical focusing nonlinear Schrödinger equations in one dimension. J. Amer. Math. Soc., 19(4):815-920, 2006.

[47] Man Kam Kwong. Uniqueness of positive solutions of $\Delta u-u+u^{p}=0$ in $\mathbf{R}^{n}$. Arch. Rational Mech. Anal., 105(3):243-266, 1989.

[48] Jeremy Marzuola, Jason Metcalfe, and Daniel Tataru. Strichartz estimates and local smoothing estimates for asymptotically flat Schrödinger equations. arXiv preprint arXiv:0706.0544, 2007.

[49] Jeremy L Marzuola, SG Raynor, and Gideon Simpson. Nonlinear bound states in a Schrödinger-Poisson system with external potential. SIAM Journal on Applied Dynamical Systems, 16(1):226-251, 2017.

[50] Jeremy L Marzuola and Gideon Simpson. Spectral analysis for matrix hamiltonian operators. Nonlinearity, 24(2):389, 2010.

[51] Kevin McLeod and James Serrin. Uniqueness of positive radial solutions of $\Delta u+f(u)=0$ in $\mathbf{R}^{n}$. Arch. Rational Mech. Anal., 99(2):115-145, 1987.

[52] K. Nakanishi and W. Schlag. Global dynamics above the ground state energy for the cubic NLS equation in 3D. Calc. Var. Partial Differential Equations, 44(1-2):1-45, 2012.

[53] Kenji Nakanishi. Global dynamics above the first excited energy for the nonlinear schrödinger equation with a potential. Communications in Mathematical Physics, 354(1):161-212, 2017.

[54] Kenji Nakanishi. Global dynamics below excited solitons for the nonlinear schrödinger equation with a potential. Journal of the Mathematical Society of Japan, 69(4):1353-1401, 2017.

[55] Galina Perelman. Asymptotic stability of multi-soliton solutions for nonlinear Schrödinger equations. Comm. Partial Differential Equations, 29(7-8):1051-1095, 2004.

[56] Peter Petersen. Riemannian geometry, volume 171 of Graduate Texts in Mathematics. Springer, Cham, third edition, 2016.

[57] Claude-Alain Pillet and C. Eugene Wayne. Invariant manifolds for a class of dispersive, Hamiltonian, partial differential equations. J. Differential Equations, 141(2):310-326, 1997.

[58] Martin Schechter. Principles of functional analysis, volume 36 of Graduate Studies in Mathematics. American Mathematical Society, Providence, RI, second edition, 2002.

[59] W. Schlag. Stable manifolds for an orbitally unstable nonlinear Schrödinger equation. Ann. of Math. (2), 169(1):139-227, 2009.

[60] Wilhelm Schlag, Avy Soffer, and Wolfgang Staubach. Decay for the wave and Schrödinger evolutions on manifolds with conical ends. I. Trans. Amer. Math. Soc., 362(1):19-52, 2010.

[61] Jalal Shatah and Walter Strauss. Instability of nonlinear bound states. Comm. Math. Phys., 100(2):173190, 1985.

[62] Barry Simon. Operator theory. A Comprehensive Course in Analysis, Part 4. American Mathematical Society, Providence, RI, 2015.

[63] A. Soffer and M. I. Weinstein. Multichannel nonlinear scattering for nonintegrable equations. Comm. Math. Phys., 133(1):119-146, 1990.

[64] A. Soffer and M. I. Weinstein. Multichannel nonlinear scattering for nonintegrable equations. II. The case of anisotropic potentials and data. J. Differential Equations, 98(2):376-390, 1992.

[65] Walter A. Strauss. Existence of solitary waves in higher dimensions. Comm. Math. Phys., 55(2):149-162, 1977. 
[66] Catherine Sulem and Pierre-Louis Sulem. The nonlinear Schrödinger equation, volume 139 of Applied Mathematical Sciences. Springer-Verlag, New York, 1999. Self-focusing and wave collapse.

[67] Susanna Terracini, Nikolay Tzvetkov, and Nicola Visciglia. The nonlinear Schrödinger equation ground states on product spaces. Anal. PDE, 7(1):73-96, 2014.

[68] Gerald Teschl. Mathematical methods in quantum mechanics, volume 157 of Graduate Studies in Mathematics. American Mathematical Society, Providence, RI, second edition, 2014. With applications to Schrödinger operators.

[69] Tai-Peng Tsai and Horng-Tzer Yau. Asymptotic dynamics of nonlinear schrödinger equations: Resonance-dominated and dispersion-dominated solutions. Communications on Pure and Applied Mathematics: A Journal Issued by the Courant Institute of Mathematical Sciences, 55(2):153-216, 2002.

[70] Michael I. Weinstein. Nonlinear Schrödinger equations and sharp interpolation estimates. Comm. Math. Phys., 87(4):567-576, 1982/83.

[71] Michael I. Weinstein. Modulational stability of ground states of nonlinear Schrödinger equations. SIAM J. Math. Anal., 16(3):472-491, 1985.

[72] Michael I. Weinstein. Lyapunov stability of ground states of nonlinear dispersive evolution equations. Comm. Pure Appl. Math., 39(1):51-67, 1986.

Department of Mathematics, Emory University, Atlanta, Ga 30322, USA

E-mail address: dborthw@emory.edu

Universität Wien, Fakultät für Mathematik, Oskar-Morgenstern-Platz 1, 1090 Vienna, Austria

E-mail address: roland.donninger@univie.ac.at

University of Basel, Department of Mathematics and Computer Science, Spiegelgasse 1, CH-4051 BASEL, Switzerland

E-mail address: enno.lenzmann@unibas.ch

Department of Mathematics, University of North Carolina, Chapel Hill, CB \#3250 Phillips Hall, Chapel Hill, NC 27599, USA

E-mail address: marzuola@math.unc.edu 